\title{
Control-oriented modeling and adaptive parameter estimation of a Lithium ion intercalation cell
}

by

\section{Pierre $\mathrm{Bi}$}

Submitted to the Department of Mechanical Engineering in partial fulfillment of the requirements for the degree of

Master of Science in Mechanical Engineering at the

\section{MASSACHUSETTS INSTITUTE OF TECHNOLOGY}

June 2015

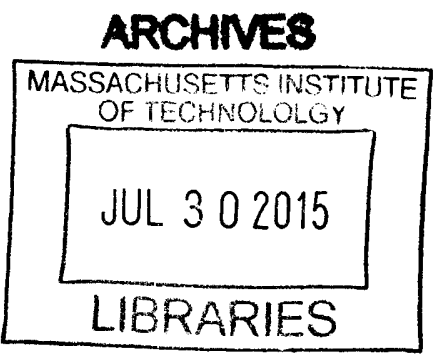

(c) Massachusetts Institute of Technology 2015. All rights reserved.

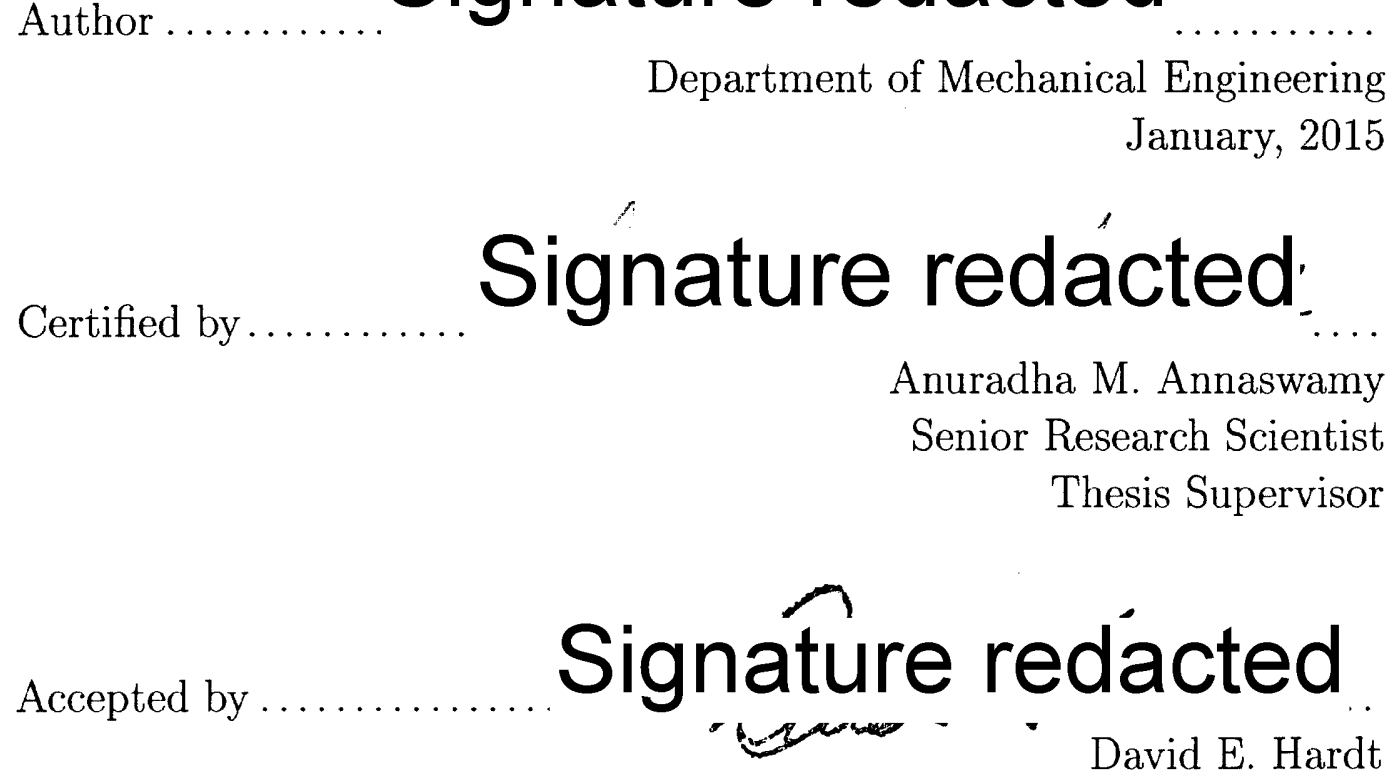

\section{Signature redacted}

Department of Mechanical Engineering January, 2015

Chairman, Department Committee on Graduate Students 


\title{
Control-oriented modeling and adaptive parameter estimation \\ of a Lithium ion intercalation cell
}

\author{
by \\ Pierre Bi \\ Submitted to the Department of Mechanical Engineering \\ on January, 2015, in partial fulfillment of the \\ requirements for the degree of \\ Master of Science in Mechanical Engineering
}

\begin{abstract}
Battery management systems using parameter and state estimators based on electrochemical models for Lithium ion cells, are promising efficient use and safety of the battery. In this thesis, two findings related to electrochemical model based estimation are presented - first an extended adaptive observer for a $\mathrm{Li}$-ion cell and second a reduced order model of the Pseudo Two-Dimensional model. In order to compute the optimal control at any given time, a precise estimation of the battery states and health is required. This estimation is typically carried out for two metrics, state of charge (SOC) and state of health (SOH), for advanced BMS. To simultaneously estimate $\mathrm{SOC}$ and $\mathrm{SOH}$ of the cell, an extended adaptive observer, guaranteeing global stability for state tracking, is derived. This extended adaptive observer is based on a non-minimal representation of the linear plant and a recursive least square algorithm for the parameter update law. We further present a reduced order model of the Pseudo Two-Dimensional model, that captures spatial variations in physical phenomena in electrolyte diffusion, electrolyte potential, solid potential and reaction kinetics. It is based on the absolute nodal coordinate formulation (ANCF) proposed in [28] for nonlinear beam models. The ANCF model is shown to be accurate for currents up to $4 \mathrm{C}$ for a $\mathrm{LiCoO}_{2} / \mathrm{LiC}_{6}$ cell. The afore mentioned extended adaptive observer is also applied to the ANCF model and parameters are shown to converge under conditions of persistent excitation.
\end{abstract}

Thesis Supervisor: Anuradha M. Annaswamy

Title: Senior Research Scientist 


\section{Acknowledgments}

Writing this thesis, I delved into the depths of adaptive control theory and electrochemistry, two fields prior unknown to me. This undertaking could not have been possible without the help of my advisor Anuradha Annaswamy, who has shown me the power and multi-facetted possibilities of adaptive control. I enjoyed the extensive discussions on adaptive observers with you and would like to thank you for the confidence you have put in me. I also would like to thank Aleksandar Kojic, Nalin Chaturvedi and Ashish Krupadanam, who have shown me the applied side of my work.

I could not have written this thesis without the constant support of my family members Thomas, Cathi, Françoise and Junqing, who always welcomed me back in Switzerland and visited me several times. My girlfriend Paola gave me the needed love and happiness to survive even the coldest days in Cambridge. Finally, I want to thank Florent, Shuo and Claire, with whom I have shared great moments at MIT and who will stay friends for a lifetime. 


\section{Contents}

$\begin{array}{lll}1 & \text { Introduction } & 9\end{array}$

2 Battery Management Systems $\quad 13$

2.1 Definition and tasks of a battery management system . . . . . . 13

2.2 Battery Monitoring Systems . . . . . . . . . . . 16

2.2 .1 State and parameter estimation methods. . . . . . . . 18

3 Adaptive estimation of parameters and states of a Lithium ion cell 25

3.1 Adaptive observer - Nonminimal representation II . . . . . . . . . 26

3.1.1 Recursive Least Square method . . . . . . . . . . . . . . 31

3.1.2 Extension of the nonminimal representation II for plants with direct feedtrough .................. 33

3.2 Adaptive observer for a single particle model plant . . . . . . . . . 35

3.2.1 The Single Particle Model . . . . . . . . . . . . . . . . 35

3.2.2 Simplified SPM: Volume averaged projections and linearized

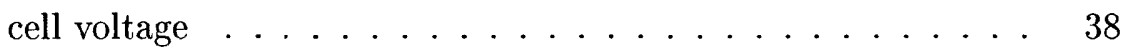

3.2.3 States and parameters of interest . . . . . . . . . . 40

3.2 .4 Observability and stability of the SPM . . . . . . . 41

3.2.5 Simulation setup and results ............. 43

3.3 Adaptive estimator for a two cathode material plant . . . . . . . . 50

3.3.1 The Single Particle Model for a cathode with two insertion ma-

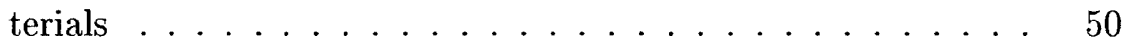

3.3.2 Simplified model for two cathode materials . . . . . . . . 52 
3.3 .3 Simulation setup and results ............ 56

4 Control-oriented electrochemical lithium ion cell models 61

4.1 Pseudo two dimensional model . . . . . . . . . . . . . . . . . 62

4.1.1 Current in the electrode . . . . . . . . . . . . . 64

4.1 .2 Potential in the solid electrode . . . . . . . . . . . 65

4.1.3 Potential in the electrolyte of the electrode . . . . . . 6 65

4.1.4 Transport in the electrolyte of the electrode . . . . . . 66

4.1.5 Transport in the solid electrode ............ 67

4.1.6 Reaction kinetics of the electrode . . . . . . . . . 68

4.1.7 Governing equation in the separator . . . . . . . . . . . 69

4.2 The ANCF Li-ion cell model . . . . . . . . . . . . . . . . . 69

4.2.1 The absolute nodal coordinate formulation . . . . . . . . 70

4.2.2 The Method of Weighted Residual . . . . . . . . . 72

4.2.3 Transport in the electrolyte ............ 73

4.2 .4 Transport the solid electrode . . . . . . . . . 75

4.2.5 Reaction kinetics at the electrode . . . . . . . . 76

4.3 Summary of the ANCF model . . . . . . . . . . . . . . . 79

4.4 Validation of the ANCF model and comparison to SPM . . . . . 80

4.4.1 Comparison of the ANCF model to the SPM . . . . . . . . 85

4.4.2 Extended adaptive observer on a ANCF model plant . . . . 86

$\begin{array}{llr}5 & \text { Conclusion } & 89\end{array}$

$\begin{array}{lr}\text { A } & 93\end{array}$

A.1 Notations . . . . . . . . . . . . . . . . . . . . 94

A.2 Parameter values . . . . . . . . . . . . . . 96

A.3 Input signal . . . . . . . . . . . . . . . . . . 97 


\section{Chapter 1}

\section{Introduction}

In march 2012, US president Obama launched the "EV Everywhere Grand Challenge" as one of the Clean Energy Grand Challenges. Goal of this initiative is to provide plug-in electric vehicles to the US population at the same price as gasoline-fueled vehicles by 2022 . This initiative aims to provide a sustainable solution to transport systems by decreasing dependency of the US on foreign oil, improve the competitive position of U.S. industry, create jobs through innovation and diminish the US' impact on pollution and global warming. To reach these goals, the technology in electric vehicles have to overcome major economic and technological hurdles. One major concern is the development of an electrical energy storage, that is affordable and can reach the high energy density and specific energy of gasoline. Lithium, which is the third lightest element and has the highest oxidation potential of all known elements, is therefore an ideal candidate for battery technology in electric vehicles.

Although successful market entries of battery powered vehicles, such as the Nissan

Leaf, Chevy Volt and Tesla S, occurred, current Li-ion battery technology has yet to establish itself as primary medium for energy storage in electric vehicles. Pertaining problems are related to low specific energy, safety issues and high costs. For example, a recent study by EPRI revealed that of all survey respondents that planned to buy a new vehicle before the end of 2013 , "only $1 \%$ indicated that they were willing to make a PEV purchase given the premium prices". Safety issues of lithium batteries, 
demonstrated in recent vehicle accidents of Tesla $\mathrm{S}$ models [22], can further restrain the mass market from buying electric vehicles. In response to these pertaining issues, research on Lithium batteries has been significantly growing over the last years, as can be inferred from historical data on patent filings. Thus, innovation could help to overcome technical and economic barriers. Current research focuses on either pushing the technical properties of lithium ion batteries by finding new materials and designs or by determining efficient use of current technology. Battery management system accomplish the latter. They enable efficient use battery packs, while preserving safety measures required by the automobile sector.

A sophisticated battery management system (BMS), enables optimal use of batteries in electric vehicles, while ensuring safety for the user. It further ensures maximal power output, long driving ranges and fast charging possibilities, without compromising the batteries lifetime. In order to compute the optimal control at any given time, a precise estimation of the battery states and health is required. This estimation is typically carried out for two metrics, state of charge (SOC) and state of health (SOH), for advanced BMS. SOC is the equivalent of a fuel gauge and defined by the ratio between residual battery capacity and total nominal capacity. Available power is defined by the maximum power that can be applied to or extracted from a cell at a given instant and the predicted power available for a given horizon. $\mathrm{SOH}$ indicates the condition of the battery compared to its original condition. These battery states and parameters can not be directly measured in a non-laboratory environment and therefore, have to be inferred from the available cell measurements. In this thesis, we present two new findings on battery states and parameters estimation, the first is a new reduced order model, called ANCF model and the second is an adaptive observer for Li-ion cells.

All estimation softwares rely on a mathematical model describing the battery. Depending on the modeling approach, different types and quality of information can be obtained. Estimating SOC, available power and $\mathrm{SOH}$ based on electrochemical 
models has recently gained attention [2]. Electrochemical models are based on the physical phenomena of the cell and can replicate with high accuracy the li-ion cell behavior in any operation mode. However, due to the mathematical complexity of those models, computation effort is considerably higher for simulating them. In addition, the derivation of suited estimators becomes more complex. Thus, only a certain subset of electrochemical model with minimal computational effort and simple mathematical representations are feasible for online estimation schemes. A simple model, called the single particle model (SPM), has been proposed by different authors [2] [18]. It can be applied to any intercalation material based cell and incorporates the cell's dominant physical phenomena, like Lithium ion diffusion through the electrode and electrochemical reaction kinetics at the surface of the electrode material. The model is a reduced version of the original first principle model proposed by Newman et. al [13], a widely accepted physical representation of the cell. In this paper, we present a simple, yet more detailed model relative to the SPM, that captures spatial variations in physical phenomena in electrolyte diffusion, electrolyte potential, solid potential and reaction kinetics. This model is based on the absolute nodal coordinate formulation (ANCF) proposed in [28] for nonlinear beam models. The specific advantages of the ANCF model over those in [2] [18] come from its ability to capture the cell behavior at high currents, that may be of the order of several Cs. The underlying approximation is based on spatial discretization elements with third-order polynomials as basis functions.

With the above ANCF model as starting point, the next problem to be addressed is state and parameter estimation. In this thesis, we carry out the first step of this estimation. In this step, we begin with the structure of the SPM model and estimate its parameters and states using an adaptive observer [12]. The ANCF model is then used to validate this estimation by utilizing the former as an evaluation model. Estimators based on the SPM model, have shown great potential. Santhanagopalan et al. [18] showed that they were able to track SOC of a Sony 18650 Cell applying a Kalman Filter based on the single particle model within $2 \%$ error boundaries. 
They simulated the single particle model on a drive cycle of a hybrid electric vehicle for a conventional 18650 battery and showed precise tracking of SOC at low to medium currents. Observers and Kalman filters have been used for state estimation in electrochemical models in [26] [18] and observers [26] as well, but these studies assume perfect knowledge of parameters. Li-ion cell properties may be obtained a priori through experimental test, but these start to alter over time due to various aging phenomena. Total capacity of the battery pack can change up to $20 \%$ over the whole lifetime and have to be tracked precisely. Hence, a parameter estimation algorithm running parallel to the state estimation has to be implemented. Providing such a system with simultaneous state and parameter estimation is difficult, as one depends on the other and thus, imprecise estimation in one propagates to the other. The adaptive observer, first introduced by [8], is therefore an appropriate tool for simultaneous state and parameter estimation, and is carried out in this paper. With a simple extension of results in [12] and a recursive least square (RLS) parameter update schemes [8], we are able to develop a stable adaptive observer that can be shown to lead to parameter estimation with conditions of persistent excitation.

This work is structured as follows. In chapter two, we introduce the battery management system and give a brief overview on its different tasks. We will show that the battery monitoring system is a key part of a BMS and that other features are dependent on it. Furthermore, we will give a review on the suggested estimation algorithm for monitoring systems and suggest future topics of interest for this technology. In the third chapter, we present the application of adaptive observers to different cell models. We start by deriving the generic form of the observer and then apply it to the single particle model with one electrode material on each side. To show the application of the adaptive observer to higher order models, we also implement it on a two cathode material cell. The last chapter introduces the ANCF model and reviews the performance of the extended adaptive observer, if applied to the ANCF model. A short introduction to the porous electrode model is added in the same chapter for reason of completeness. 


\section{Chapter 2}

\section{Battery Management Systems}

This chapter introduces the purpose and structure of a battery management system for electric vehicles. Special emphasis is put on the battery monitoring system, which performs parameter and state estimation of battery cells. Although the battery monitoring system is a small piece of the whole management system, its central role in an advanced BMS will become apparent. We will analyze the state-of-the-art implementation and summarize various research efforts made in this field in section 2.1. Different cell monitoring solutions are presented in section 2.2. Furthermore, we elaborate on possible future work in this field in section 2.2.1.

\subsection{Definition and tasks of a battery management system}

A battery management system (BMS) is composed of hardware and software that enable the efficient use of a battery pack, without compromising its safety and lifespan. Efficient use of a battery is characterized by high energy and power extraction/insertion. A BMS capable of optimizing those features may increase e.g. the driving range and acceleration, optimize regenerative braking and enable faster charging of BEVs and HEVs. At the same time, a battery management system has to ensure safe operation of the battery and guarantee the specified lifespan of the battery. 
Safety which is a major concern for current battery technology. Whenever thermal runaway of a battery cell or pack occurs, severe damages to humans and technology can be the consequence. To prevent such situations the BMS has keep the battery pack in a tight operation window, defined by maximum/minimum temperatures and maximum/minimum charge states. To fulfill these afore mentioned tasks, different software and hardware modules have to be included in the BMS. Amongst others, these modules fulfill following functions [10] [2]:

Data acquisition: This module is in charge of monitoring measurements of individual cell and battery pack voltage, current and temperature. Additional functions might include e.g.recognition of smoke, collision and gases. Depending on cell chemistry voltage measurements have to precise, i.e. for electrode materials which exhibit very flat open-circuit voltage versus state of charge curves higher precision is required [15] [10]. Unprecise voltage and current measurements propagate into wrong state and parameter estimations by the battery monitoring system. Accuracy targets for current measurements are $0.5 \%$ to $1 \%$ and for voltage measurements $5 \mathrm{mV}$ [15]. Noise content of these measured signals are usually very low, which is helpful for estimation based on deterministic signals [23].

Battery Monitoring System: This unit continuously determines important battery states and parameters during operation. States of interest are state of charge and available power [23]. Parameters of interest are capacity, state of health (SOH) and remaining useful life (RUL). Unfortunately, all of the afore mentioned states and parameters of the cell can not be directly measured online. They are estimated relying on the three measurable states - voltage, current and temperature. Thus, this module has to rely on different algorithms, to keep track of them. A detailed analysis of this module is provided in the next section.

Energy Management system: This module controls charging and discharging of the battery cells for various scenarios. Based on the available power estimation it de- 
termines the optimal acceleration of the BEV or HEV, which will not exceed the operational window of the lithium ion cell. Furthermore, it can optimize control energy recuperation and dissipation by braking during deceleration [23]. Charging patterns are also controlled by this unit. Finally, it also performs battery equalization. In order to maintain a high capacity for the whole battery pack, all cells need to have the same state of charge [9]. Otherwise, capacity of the battery pack is determined by the cell with lowest SOC during discharging and vice versa by the cell with highest SOC during charging. Imbalance in cells occur due to external and internal sources [1]. Internal sources of imbalance are caused by differences in cell impedance and self-discharge rate. External sources are either unequal current draining or temperature gradients across the battery pack. Active cell balancing requires an external circuit in combination with power electronics, which can transfer energy between the cells.

Thermal control: This module keeps the battery temperature within the operation range for which safety is guaranteed. Holding temperature at an optimal point also prevents fast degradation of capacity and internal resistance of the cells. Both cooling at high temperatures and warming at low temperatures can be necessary depending on battery chemistry. Depending on the cell geometry temperature profiles and heat transfer vary considerable. Laminated cells offer a high heat exchange surface and exhibit small temperature gradients, therefore air cooling is applied [15]. Whereas, 18650 cells usually require liquid coolant. Thus, thermal control systems have to be adapted to the respective cell and package design.

Safety management: A fault recognition and diagnosis system is included in every battery management system. Deviations from operating conditions, such as deep discharge, overcharge, excessive temperatures and mechanical failures are recognized by this unit. Mitigation plans, such as cutting off battery power supply, to prevent further damage to battery and user are operated by this module if special events are detected. It also informs the battery user about end of battery lifetime and needs for 
maintenance.

The battery monitoring system is the centerpiece of an advanced battery management system. Optimal strategies for cell balancing are based on accurate estimation of individual cell SOC, discharge and charge control can not be efficiently executed without precise knowledge of available power and thermal control can only be optimized if the charging pattern is a priori known. It is therefore imperative to obtain precise estimates of the different states of a battery. Although industrial and academic research have put great effort into developing advanced monitoring system, this technology is still considered to be premature [27] at this stage. We therefore provide a critical review on the status quo of monitoring methods in the next section.

\subsection{Battery Monitoring Systems}

Aside from the measured voltage, current and temperature signals, a variety of other cell states and parameters are needed for the optimal operation of a battery pack. Waag et al. [23] identified state of charge (SOC), capacity, available power, impedance, state of health (SOH) and remaining useful life (RUL) as main states and parameters

of interest. SOC is the equivalent of a fuel gauge, indicating the energy left in the cell. It is the ratio between residual battery capacity and total nominal capacity of a cell expressed in percentage. Nominal capacity is measured by charging and discharging the unused battery between specified voltage limits and constant currents. Over the whole battery lifetime the real capacity, which is the total available energy when the battery is fully charged, will deviate from the nominal state due to material aging. Available power is defined by the maximum power that can be applied to or extracted from cell at a given instant and the predicted power available for a given horizon. The length of the prediction horizon is dependent on the control algorithm deployed in the energy management system and is usually between $1 s$ and 20s [23]. Internal cell power losses are caused by diffusion, reactions kinetics and side reactions, which can are usually referred to as internal impedance. $\mathrm{SOH}$ usually refers to the 
condition of the battery compared to its original condition. Depending on the battery application, variations in the definition of $\mathrm{SOH}$ appear. For hybrid electric vehicle, which have high power requirements, $\mathrm{SOH}$ is generally defined by the power capability (internal impedance). For battery electric vehicles on the other hand $\mathrm{SOH}$ is usually a compound indicator of capacity and internal resistance of a cell [10]. 100\% $\mathrm{SOH}$ represents a new cell after manufacturing and $0 \%$ a cell when it reaches its end of lifetime, e.g. when the capacity reaches $80 \%$ of its nominal value or the internal impedance increased by a certain factor. RUL refers to the number of load cycles or time until $0 \% \mathrm{SOH}$ is attained.

Compared to applications in consumer electronics, technical requirements defined for lithium ion batteries in electric vehicles are more demanding and stringent. Several technical requirements, as e.g. prescribed by the US Advanced Battery Consortium (USABC), make Lithium ion cell monitoring a challenging task. Battery packs in electric vehicles have to be functional for a lifetime of ten or more years and bear more than 1000 cycle at $80 \%$ SOC. In addition, they have to bear a wide range of operation modes influenced by external factors, like e.g. extreme temperatures, aggressive driving or changing charging patterns. Internal parameters change substantially on both time scales. During operation, like e.g. a short drive, they alter due to changing SOC and temperature. On the long run, they alter substantially due to aging and cycling effects. Hence, both variations have to be precisely identified by the monitoring system. Beside guaranteeing precise tracking, constraints have also to be considered by the monitoring system. To guarantee safety, battery manufacturers define operation windows for maximum and minimum temperature, voltage, and currents of a cell. These specifications prevent e.g. side reactions due to excess voltages or accelerated aging due to elevated temperatures. However, it also puts tight constraints on the prediction of available power and energy and complicates their calculation. Finally, characteristics and limits of hardware have also to be considered. Imprecise inputs, such as offsets and noise in voltage and current measurements are present due to sensor limits. Furthermore, algorithms have to optimized not to exceed 
the computational limits of the MCUs. These requirements and constraints make it therefore difficult to design precise and sophisticated monitoring algorithms, which can be readily implemented on current hardware. In the following, we will describe and analyze the status quo of parameter and state estimation for Lithium ion cells.

\subsubsection{State and parameter estimation methods.}

Available power of a lithium ion cell is determined by a multitude of physical phenomena. These include amongst other, reaction kinetics, electrolyte diffusion, solid electrode diffusion and side reactions. They are all characterized by complex dynamics and relationships. Capturing all those effects in order to predict available power for a time period of 1 to $20 \mathrm{~s}$ is further complicated by constraints set from the cell safety operation window. Methods for estimating available power are based either on equivalent circuit or electrochemical models.Capacity fade can be attributed to loss of recyclable lithium due to side reactions and loss of active material on both electrodes [16]. Internal impedance increase is due to aging phenomena. These parameters can be estimated by either using predetermined models, which have been obtained in experiments or by online parameter identification methods. We will focus on online identification methods, which can account for individual cell differences.

Current integral, Ampère-hour counting: This method relies on the assumption that input current is precisely measured and capacity is a priori known. It applies a simple current integration

$$
S O C=S O C_{0}-\frac{1}{C} \int_{t_{0}}^{t} f I \mathrm{~d} \tau
$$

where $I$ is the applied current, $C$ the capacity, $f$ the coulombic efficiency and $S O C_{0}$ the initial value, to estimate SOC. The coulombic efficiency factor describes the charge lost due to parasitic reactions, such as the formation of a solid electrolyte interface (SEI) on anode side. The ampère-hour provides a simple estimation method for short operations. However, the estimated SOC can deteriorate over longer periods of time due to imprecise current measurements. Hence, $S O C_{0}$ has to be recalibrated at a 
timescale $\tau_{2} \gg \tau$. Open circuit voltage based estimation is often used as recalibration method. Even in combination with open circuit voltage based calibration, such a method is not reliable, if capacity factor and coulombic efficiency are not precisely known [18] [10]. Both parameters are affected by aging and have to be precisely tracked.

Open circuit voltage based estimation: This estimation method exploits the well defined relation between OCV and SOC, which can be empirically determined and is described by OCV-curves. Open circuit voltage is defined as the potential difference between the two electrodes, when no current is applied to the cell and all internal states are at rest. Hence, OCV can be measured, when the cell is shut off and relaxation processes have taken place. In contrast to ampère-hour counting, where estimation in imprecise if battery parameters drift, OCV-based estimation stays reliable over longer periods of time. In fact, it has been shown that the characteristic OCV-SOC relation does not significantly drift over time. However, this method is bound by the assumption that there exists phases of long rests, where the battery returns to a balanced state. Voltage relaxation can be mainly attributed to slow diffusion processes in the solid and electrolyte. These phases of relaxation can extend over several hours, as e.g. for $\mathrm{C} / \mathrm{LiFePO}_{4}$ which fully relax after three or more hours at low temperatures [10]. It has been suggested by different authors [] [I to estimate the relaxation curves online and then deduct the OCV from the voltage measurement before relaxation has taken place. Relaxation models were derived by an empirical approach and did not base on a physical interpretation. Those empirical models are usually bound to the specific cell chemistry and dimension.

Artificial neural network models or fuzzy logic algorithms: For these methods, the lithium ion cell is modeled by artificial neural networks. No precise knowledge on the physical behavior is needed, but an extensive set of past data of the cell signals is needed to train the ANNs. The physical insight provided by electrochemical models is not available in this case. Furthermore, these algorithms usually require high 
computational effort.

Equivalent Circuit Models (ECM) based state and parameter estimation: A common estimation approach, is to approximate the cell dynamics by an equivalent circuit model and estimate the modeled states. Equivalent circuit models are lumped parameter models [25]. Cell characteristics are approximated by a combination of RC circuits. Most ECM for monitoring systems are restricted to one or two RC elements due to the computational limits of the MCUs [24]. Depending on cell chemistries and design, ECMs have different layouts. Reactions kinetics described by Butler-Volmer equations in electrochemical models are approximated by a charge transfer resistance $R_{t c}$. Diffusion processes and double-layers can be modeled by RC elements [4]. Impedances have constant values and are independent of other states, such as SOC, current and temperature. They are updated depending on the current operation with either predefined look up tables or online estimation methods. A series of works focus on state estimation solely, assuming parameters to be a priori known. Suggested estimation algorithms are amongst others, Kalman filters, Particle filters, recursive least square filters, Luenberger observers, Sliding mode observers and Adaptive observers [23]. Domenico et. al [4] presented a SOC estimation based on an extended Kalman filter. Reaction kinetics were modeled by a charge transfer resistance and diffusion by a Warburg impedance, which is represented by a first order transmission line in the time domain. An additional OCV based estimation was used to estimate initial conditions. They tested the filter on a $\mathrm{LiFEPO}_{4} / \mathrm{C}$ cell of $\mathrm{A} 123$. When simulated on the battery model, assuming battery parameters to be known, estimations stayed within a $3 \%$ error boundary. For simulation on real experimental data, weighting matrices of the Kalman filter had to be updated depending on the SOC range for better convergence. They showed that SOC estimations had less than $3 \%$ deviation from the open loop model state. A disadvantage of Kalman filters is the declined performance when noise characteristics are not precisely known. Furthermore, results of a robustness analysis showed that parameter uncertainties impacted state estimation strongly. Small parameter errors of $10 \%$ could already result in $200 \%$ estimation er- 
ror. Due to the aging effects of batteries, parameters drift easily by $20 \%$ over the whole battery lifetime. In fact, considering parameter values constant or updating according to a look up table, considerably reduces robustness for any of the afore mentioned state estimation algorithm. Joint parameter and state estimation based on ECMs, were presented by [25] and [24].

A major drawback of ECMs is that their "theoretical basis [...] is based on the response of the battery to a low-amplitude ac signal" [2]. Application of this method to EVs, where the battery is exposed to more aggressive charging and discharging patterns, will not yield precise power estimations. Therefore, batteries are not used at their limits by the control algorithms, which rely on the state estimates.

Electrochemical Models based SOC state and parameter estimation: Algorithms based on electrochemical models are being recently proposed by Chaturvedi et al [2] [3]. Unlike ECMs, electrochemical model accurately capture internal processes at various operating conditions. A drawback of electrochemical models is their nonlinearity and high order, which require increased computational efforts. It is therefore imperative to find models capable of capturing the main dynamics at

Most estimation methods proposed so far are based on the single particle model (SPM). The single particle model is a reduced order model, derived from the Pseudo 2-Dimensional model [13]. Both electrodes are assumed to be single spherical particles with volume and surface equal to the active electrode material. The SPM captures diffusion of lithium ions in the solid electrodes, but assumes diffusion processes within the electrolyte as negligible. These assumptions restrict the model to current application below $1 C$. The model can be easily extended to include degradation of the electrode loading, side reactions or non isothermal behavior [16]. More details on the single particle model will be provided in Chapter 3.2.1. Estimation methods based on the single particle model or an extension of it, are extended Kalman filters (EKF), unscented Kalman filters (UKF) [16], iterated extended Kalman Filter [26] and nonlinear 
PDE back-stepping observers [11]. Extended Kalman filters proposed by [18] linearize the system at every time step by using Taylor series around the operating point. As shown by [16] the state estimates for the single particle model, which exhibits strong nonlinearities, don't converge due to errors caused by the linearization. As alternative, the UKF method has been developed, which is a derivative free filtering method. Estimation is performed by approximating the probability distribution function (pdf) of the voltage output through nonlinear transformation of the state variables pdfs. To estimate capacity fade and SOC of the cell, Rahimian et al. included degradation electrode loading and material formation through side reactions as additional states. Hence, capacity and SOC were estimated simultaneously as states by the UKF. The UKF was tested on synthetic data obtained from the nonlinear SPM model under Low Earth Orbit cycling conditions, which are usual for satellites. All capacity loss mechanism and the SOC were tracked satisfactorily. No simulations on real data were provided. A different approach to estimate parameters and states simultaneously is presented by [26], who applied a nonlinear adaptive observer to a simplified single particle model. They simplified the single particle model by approximating the solid diffusion equations with a volume-averaged projection as a first order system, whose state is the volume averaged lithium concentration in the electrode $\bar{c}_{s}$. In addition, they modeled the OCV as a natural logarithmic function, which is priorly fitted to the OCV data. Based on these simplifications, a smart choice of state transformation allows them to transform the plant description into the form

$$
\begin{aligned}
& \dot{z}=A(\theta) z+\phi(z, u, \theta) \\
& y=C z
\end{aligned}
$$

where $z \in \mathbb{R}^{n}$ is the state vector, $\theta \in \mathbb{R}^{m}$ the unkown parameters, $u$ the input and $y$ the output. For bounded signals $z(t), u(t)$, bounded parameters $\theta$ and $\phi(z, u, \phi)$ being Lipschitz with respect to $z$ and $\phi$, stability for the following adaptive observer 
can be shown

$$
\begin{aligned}
& \dot{\hat{z}}=\gamma(y-\hat{y})+\hat{\phi}+\gamma \Gamma \dot{\hat{\theta}} \\
& \dot{\Gamma}=-\gamma \Gamma+\frac{\partial \hat{\phi}}{\partial \hat{\theta}} \\
& \dot{\theta}=\gamma P \Gamma^{T}(y-\hat{y}) \\
& \dot{P}=-\gamma P \Gamma^{T} \Gamma P+\gamma P .
\end{aligned}
$$

They validated the adaptive observer on the simplified plant model for two unknown parameters. Parameters and states were proven to converge for a persistently exciting signal. Application to a real system was not shown. One disadvantage of this estimator is the low order approximation for the solid diffusion, which significantly deviates from the PDE solution at higher currents [21]. A transformation for higher order systems is not immediately apparent. In addition, the approximation of OCV by a generic logarithmic function would have to be validated for different cell chemistries.

As mentioned, electrochemical models used for battery monitoring have been mainly based on the single particle model. Although, the model is derived from the highly accurate porous electrode model [13], it can not reliably capture internal dynamics of batteries subjected to $\mathrm{EV}$ drive cycles. Input currents of a battery can reach up to $3 C$ for peak values during a drive cycle. Neglected dynamics in the single particle model, such as the electrolyte diffusion, become apparent at these peaks. Thus, if future estimation algorithms have to rely on electrochemical models, relaxations on the assumptions made in the SPM are necessary. These battery models have validated on drive cycles with currently used battery chemistries. Validation of battery estimation methods have also to be performed on real battery data. 


\section{Chapter 3}

\section{Adaptive estimation of parameters and states of a Lithium ion cell}

When estimating the internal states and parameters of the Lithium cell, we can only rely on two measurable signals, which are the cell current and the cell voltage. Using these two signals, we want to estimate internal states and unknown parameters simultaneously. As discussed in 2.2 we do not want to handle the state estimation and parameter estimation as two separate cases, but rather derive an observer which guarantees global tracking convergence for both. For this purpose, we present an adaptive observer, which is an extension of the original nonminimal representation presented in [12] and [8]. This adaptive observer relies on a non-minimal representation of the linear plant and can guarantee stable estimation around the operating point.

We first derive the nonminimal observer structure of [12] in section 3.1 by showing that there exist a specific non-minimal representation of linear time invariant plants, which has the equivalent input-output behavior and a formulation suitable for adaptive algorithms. We then proceed to analyze the observer stability for tracking of states and parameters when using a recursive least square algorithm as update law. In section 3.1.2, we introduce the extended observer, which is applicable to plants with proper transfer functions. After deriving the extended observer structure, we apply it to the single particle model presented in chapter 3.2.2. We show that the adaptive 
observer, when slightly modified, can manage intricacies of the li-ion cell model, such as separated timescales and a large feed-through term. Finally, to show application to higher order systems, we derive the adaptive observer for the two cathode material cell presented in chapter 3.3.1.

\subsection{Adaptive observer - Nonminimal representation II}

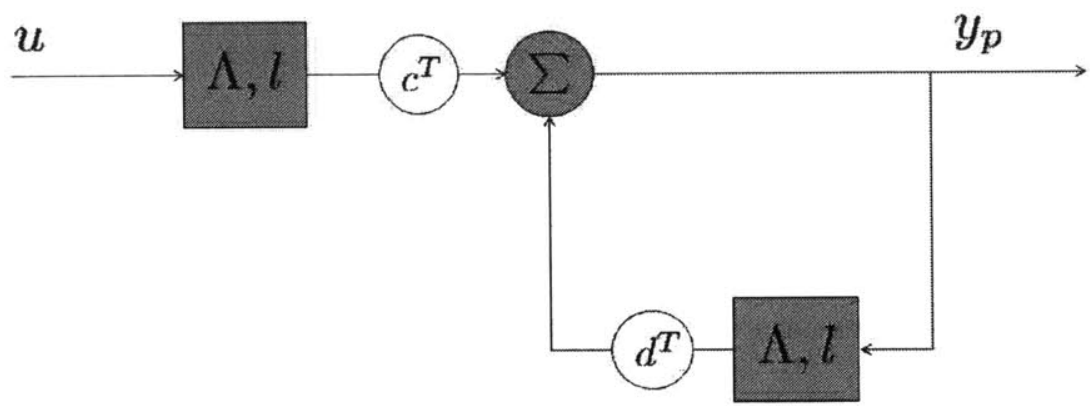

Figure 3-1: Nonminimal representation II

The adaptive observer presented in the following, was developed by [8] and is called the non-minimal representation II observer in [12]. It is suitable for any controllable and observable single-input single output $\mathrm{n}$-th order LTI system

$$
\begin{array}{r}
\dot{\mathbf{x}}_{p}=\mathbf{A} \mathbf{x}_{\mathbf{p}}+\mathbf{b} u \\
y_{p}=\mathbf{h}^{\mathbf{T}} \mathbf{x}_{\mathbf{p}} .
\end{array}
$$

For reasons of simplicity, we will assume $(\mathbf{A}, \mathbf{b})$ to be in control canonical form, i.e.

$$
\mathbf{A}=\left[\begin{array}{ccccc}
0 & 1 & 0 & \ldots & 0 \\
0 & 0 & 1 & \ldots & 0 \\
\vdots & \vdots & \vdots & \ddots & \vdots \\
0 & 0 & 0 & \ldots & 1 \\
-a_{0} & -a_{1} & -a_{2} & \ldots & -a_{n-1}
\end{array}\right] \text { and } \mathbf{b}=\left[\begin{array}{c}
0 \\
0 \\
0 \\
0 \\
1
\end{array}\right]
$$


The presented adaptive observer is based on the notion that the LTI system above can be represented by a nonminimal plant with $2 n$ states and $2 n$ parameter. We will show that the nonminimal plant has equivalent input-output behavior and its states can be mapped back to the original plant states. The suggested nonminimal plant design is shown in figure (3-1) and defined by the differential equations

$$
\begin{aligned}
& \dot{\boldsymbol{\omega}}_{1}=\boldsymbol{\Lambda} \boldsymbol{\omega}_{1}+\boldsymbol{l} u \\
& \dot{\boldsymbol{\omega}}_{2}=\boldsymbol{\Lambda} \boldsymbol{\omega}_{2}+\boldsymbol{l} y_{p} \\
& y_{p}=\boldsymbol{c}^{T} \boldsymbol{\omega}_{1}+\boldsymbol{d}^{T} \boldsymbol{\omega}_{2},
\end{aligned}
$$

where $\boldsymbol{\omega}_{1}: \mathbb{R} \mapsto \mathbb{R}^{n}, \boldsymbol{\omega}_{2}: \mathbb{R} \mapsto \mathbb{R}^{n}$ and $\mathbf{c}, \mathbf{d} \in \mathbb{R}^{n}$. The advantage of this representation is the the output structure, which is a linear combination of states. This will simplify the derivation of a suitable adaptive observer, as will be shown later on. Futhermore, $\boldsymbol{\Lambda}$ can be any arbitrary asymptotic stable matrix, where $(\boldsymbol{\Lambda}, \mathrm{l})$ is controllable. Although the structure of $(\Lambda, l)$ has significant impact on the filtering of $y$ and $u$, there is no literature on the optimal design of it. For further analysis, we choose $(\Lambda, 1)$ to be in control canonical form

$$
\boldsymbol{\Lambda}=\left[\begin{array}{ccccc}
0 & 1 & 0 & \ldots & 0 \\
0 & 0 & 1 & \ldots & 0 \\
\vdots & \vdots & \vdots & \ddots & \vdots \\
0 & 0 & 0 & \ldots & 1 \\
-\Lambda_{0} & -\Lambda_{1} & -\Lambda_{2} & \ldots & -\Lambda_{n-2}
\end{array}\right]\left[\begin{array}{c}
\omega_{1}(t) \\
\vdots \\
\omega_{n-1}(t)
\end{array}\right] \text { and } \mathbf{l}=\left[\begin{array}{c}
0 \\
\vdots \\
0 \\
1
\end{array}\right]
$$

The control canonical form of $(\Lambda, 1)$ will a simple state transformation Notice that the matrix representation of the non-minimal representation II is given by

$$
\left[\begin{array}{c}
\dot{\boldsymbol{\omega}}_{1} \\
\dot{\boldsymbol{\omega}}_{2}
\end{array}\right]=\underbrace{\left[\begin{array}{cc}
\Lambda & \mathbf{0} \\
\mathbf{l} \mathbf{c}^{T} & \boldsymbol{\Lambda}+\mathbf{l d}^{T}
\end{array}\right]}_{A_{n m}}\left[\begin{array}{l}
\boldsymbol{\omega}_{1} \\
\boldsymbol{\omega}_{2}
\end{array}\right]+\underbrace{\left[\begin{array}{l}
\boldsymbol{l} \\
\mathbf{0}
\end{array}\right]}_{b_{n m}} u(t),
$$


where $\boldsymbol{A}_{n m}$ is asymptotically stable and $\left(\boldsymbol{A}_{n m}, \boldsymbol{b}_{n m}\right)$ is controllable. In the following, we determine the parameters $\boldsymbol{c}, \boldsymbol{d}$ by comparing the transfer functions of the nonminimal and the minimal plant. We define transfer function from $u$ to $\omega_{1}$ as

$$
\frac{\Omega_{1}(s)}{U(s)}=\frac{P(s)}{R(s)}=\mathbf{c}^{T}(s \mathbf{I}-\mathbf{\Lambda})^{-1} \mathbf{l}=\frac{s^{n-1} c_{n-1}+\ldots+s c_{1}+c_{0}}{s^{n}+s^{n-1} \Lambda_{n-1}+\ldots+s \Lambda_{1}+\Lambda_{0}}
$$

and from $y_{p}$ to $\omega_{2}$ as

$$
\frac{\Omega_{2}(s)}{Y(s)}=\frac{Q(s)}{R(s)}=\mathbf{d}^{T}(s \mathbf{I}-\mathbf{\Lambda})^{-1} \mathbf{l}=\frac{s^{n-1} d_{n-1}+\ldots+s d_{1}+d_{0}}{s^{n}+s^{n-1} \Lambda_{n-1}+\ldots+s \Lambda_{1}+\Lambda_{0}} .
$$

Hence, the resulting input output behavior of the non-minimal plant is defined by

$$
W(s)=\frac{P(s)}{R(s)-Q(s)}=\frac{s^{n-1} c_{n-1}+\ldots+s c_{1}+c_{0}}{s^{n}+s^{n-1}\left(\Lambda_{n-1}-d_{n-1}\right)+\ldots+s\left(\Lambda_{1}-d_{1}\right)+\left(\Lambda_{0}-d_{0}\right)} .
$$

If we compare $W(s)$ with the plant transfer function

$$
W_{p}(s)=\boldsymbol{c}^{\boldsymbol{T}}(s \boldsymbol{I}-\boldsymbol{A})^{-1} \boldsymbol{b}=\frac{s^{n-1} b_{n-1}+s^{n-2} b_{n-2}+\ldots+s^{2} b_{2}+s b_{1}+b_{0}}{s^{n}+s^{n-1} a_{n-1}+s^{n-2} a_{n-2}+\ldots+s^{2} a_{2}+s a_{1}+a_{0}}
$$

where

$$
\begin{aligned}
& P_{b}(s)=s^{n-1} b_{n-1}+s^{n-2} b_{n-2}+\ldots+s^{2} b_{2}+s b_{1}+b_{0} \\
& P_{a}(s)=s^{n}+s^{n-1} a_{n-1}+s^{n-2} a_{n-2}+\ldots+s^{2} a_{2}+s a_{1}+a_{0},
\end{aligned}
$$

it is obvious that if

$$
\left[\begin{array}{c}
c_{0} \\
\vdots \\
c_{n-1}
\end{array}\right]=\left[\begin{array}{c}
b_{0} \\
\vdots \\
b_{n-1}
\end{array}\right] \text { and }\left[\begin{array}{c}
d_{0} \\
\vdots \\
d_{n-1}
\end{array}\right]=\left[\begin{array}{c}
-a_{0}+\Lambda_{0} \\
\vdots \\
-a_{n-1}+\Lambda_{n-1}
\end{array}\right] \text {, }
$$


$W(s)$ and equation (3.9) become equivalent. Having derived the parameters vectors, we proceed to finding the transformation matrix $\mathbf{C}$, for which

$$
\mathbf{x}_{p}=\mathbf{C x}_{n p}
$$

where $\mathbf{x}_{p}$ denote the plant states and $\mathbf{x}_{n p}$ the nonminimal states. We can find the solution for $\mathbf{C}$ by first transforming the nonminimal system (3.5) to its control canonical form. The nonminimal control canonical form can then be easily transformed back to the minimal representation. The transformation matrix for system (3.5) to control canonical form is given by

$$
\mathbf{T}=\mathbf{R M}
$$

where $\mathbf{R}$ is the controllability matrix defined by

$$
\mathbf{R}=\left[\mathbf{A}_{n m} \mathbf{b}_{n m}, \mathbf{A}_{n m}^{2} \mathbf{b}_{n m}, \ldots, \mathbf{A}_{n m}^{2 n} \mathbf{b}_{n m}\right]
$$

and

$$
\mathbf{M}=\left[\begin{array}{ccccc}
a_{0} & a_{1} & \ldots & a_{n-1} & 1 \\
a_{1} & a_{2} & \ldots & 1 & 0 \\
\vdots & \vdots & . \cdot & \vdots & \vdots \\
a_{n-1} & 1 & \ldots & 0 & 0 \\
1 & 0 & \ldots & 0 & 0
\end{array}\right]
$$

If we denote the states of the control canonical form as $\overline{\mathbf{X}}_{n m}$, the transfer functions from $u$ to $\bar{X}_{n m, i}$ are defined by

$$
\left[\begin{array}{c}
\frac{\bar{X}_{n m, 1}(s)}{U(s)} \\
\vdots \\
\frac{\bar{X}_{n m, 2 n}(s)}{U(s)}
\end{array}\right]=\left[\begin{array}{c}
\frac{1}{R(s) P_{a}(s)} \\
\vdots \\
\frac{s^{2 n-1}}{R(s) P_{a}(s)}
\end{array}\right]
$$

where $P_{a}(s)$ is the denominator of the plant transfer function as defined in Eq.(3.9). Further, the transfer function from $u$ to the states of the minimal plant are defined 
by

$$
\left[\begin{array}{c}
X_{1}(s) \\
\vdots \\
X_{n}(s)
\end{array}\right]=\left[\begin{array}{c}
\frac{1}{P_{a}(s)} \\
\vdots \\
\frac{s^{n-1}}{P_{a}(s)}
\end{array}\right]
$$

Finally, the solution for the transformation matrix $\overline{\mathbf{C}} \in \mathbb{R}^{n \times 2 n}$ for which

$$
\mathbf{x}_{p}=\overline{\mathbf{C}} \overline{\mathbf{x}}_{n m}
$$

is found to be

$$
\overline{\mathbf{C}}=\left[\begin{array}{cccccccc}
\Lambda_{0} & \Lambda_{1} & \ldots & \Lambda_{n-1} & 1 & 0 & \ldots & 0 \\
0 & \Lambda_{0} & \Lambda_{1} & \ldots & \Lambda_{n-1} & 1 & \ldots & 0 \\
\vdots & \ddots & \ddots & \ddots & \ddots & \ddots & \ddots & \vdots \\
0 & \ldots & 0 & \Lambda_{0} & \Lambda_{1} & \ldots & \Lambda_{n-1} & 1
\end{array}\right]
$$

by comparing Eq.(3.17) and Eq.(3.18). The transformation matrix in (3.13) can thus be expressed as

$$
\mathbf{C}=\overline{\mathbf{C}} \mathbf{T}^{-1}=\overline{\mathbf{C}} \mathbf{R}^{-1} \mathbf{M}^{-1} \text {. }
$$

We now choose the observer structure to be similar to the nonminimal representation II

$$
\begin{aligned}
& \dot{\hat{\boldsymbol{\omega}}}_{1}=\boldsymbol{\Lambda} \hat{\boldsymbol{\omega}}_{1}+\boldsymbol{l} u \\
& \dot{\hat{\boldsymbol{\omega}}}_{2}=\boldsymbol{\Lambda} \hat{\boldsymbol{\omega}}_{2}+\boldsymbol{l} y_{p} \\
& \hat{y}_{p}=\hat{\mathbf{c}}^{T} \hat{\boldsymbol{\omega}}_{1}+\hat{\mathbf{d}}^{T} \hat{\boldsymbol{\omega}}_{2} .
\end{aligned}
$$

This observer can be shown to be globally stable if an appropriate update law for $\hat{\theta}$ is chosen. Depending on which update law is chosen, the performance of the observer varies strongly. We will introduce the well-known recursive least square method, which produces fast converging parameter estimates. 


\subsubsection{Recursive Least Square method}

The recursive least square method is derived by minimizing the cost function

$J_{r l s}=\int_{t_{0}}^{t} \exp (-q(t-\tau)) e_{y}^{2}(t, \tau) \mathrm{d} t+\frac{1}{2} \exp \left(-q\left(t-t_{0}\right)\right)\left(\hat{\theta}(t)-\hat{\theta}\left(t_{0}\right)\right)^{T} \mathbf{Q}_{0}\left(\hat{\theta}(t)-\hat{\theta}\left(t_{0}\right)\right)$,

where $\mathbf{Q}_{0}=\mathrm{Q}_{0}^{T}>0 . \quad J_{r l s}$ is a generalization of $J_{\text {int }}$, which includes an additional

penalty on the initial estimate of $\hat{\theta}$. Furthermore, $J_{r l s}$ is convex at any time $t$ and hence any minimum at time $t$ is also the global minimum. Therefore, we obtain the parameter update law by solving

$$
\nabla J=\int_{t_{0}}^{t} \exp (-q(t-\tau)) e_{y}(t, \tau) \hat{\omega}(\tau) \mathrm{d} t+\exp \left(-q\left(t-t_{0}\right)\right) \mathrm{Q}_{0}\left(\hat{\theta}(t)-\hat{\theta}\left(t_{0}\right)\right)=0
$$

which gives us

$$
\hat{\theta}=\Gamma(t)\left(\int_{t_{0}}^{t} \exp (-q(t-\tau)) y_{p}(\tau) \hat{\omega}(\tau) \mathrm{dt}+\exp \left(-q\left(t-t_{0}\right)\right) \mathbf{Q}_{0} \hat{\theta}\left(t_{0}\right)\right)
$$

where

$$
\Gamma(t)=\left(\int_{t_{0}}^{t} \exp (-q(t-\tau)) \hat{\omega}(\tau) \hat{\omega}^{T}(\tau) \mathrm{d} t+\exp \left(-q\left(t-t_{0}\right)\right) \mathbf{Q}_{0}\right)^{-1}
$$

It can be shown [7] that $\theta$ and $\Gamma$ are the solution of the differential equations

$$
\begin{aligned}
& \dot{\hat{\theta}}=-\Gamma \hat{\omega} e \\
& \dot{\Gamma}=q \Gamma-\Gamma \hat{\omega} \hat{\omega}^{T} \Gamma, \quad \Gamma(0)=\mathbf{Q}_{0}^{-1}
\end{aligned}
$$


However, in the solution above $\Gamma$ can grow without bounds. Therefore, we have to alter the update law to

$$
\begin{aligned}
& \dot{\hat{\theta}}=-\Gamma \hat{\omega} e \\
& \dot{\Gamma}= \begin{cases}q \Gamma-\Gamma \hat{\omega} \hat{\omega}^{T} \Gamma, \quad \Gamma(0)=\mathbf{Q}_{0}^{-1} & \text { if }\|\Gamma\| \leq r_{0} \\
0, & \text { otherwise }\end{cases}
\end{aligned}
$$

To show globally stability of the RLS, we first choose the Lyapunov function candidate

$$
V=\frac{1}{2} \tilde{\boldsymbol{\theta}}^{T} \boldsymbol{\Gamma}^{\mathbf{- 1}} \tilde{\boldsymbol{\theta}}
$$

where

$$
\tilde{\boldsymbol{\theta}}=\hat{\boldsymbol{\theta}}-\boldsymbol{\theta}
$$

denotes the parameter error between the estimate and the real plant. Taking the time derivative of $V$, we obtain

$$
\dot{V}=\tilde{\boldsymbol{\theta}}^{T} \boldsymbol{\Gamma}^{-\mathbf{1}} \dot{\hat{\boldsymbol{\theta}}}+\frac{1}{2} \tilde{\boldsymbol{\theta}}^{T} \frac{d}{d t} \boldsymbol{\Gamma}^{-1} \tilde{\boldsymbol{\theta}}
$$

If we choose the update law to be the recursive least square algorithm with output error

$$
e=\hat{y}_{p}-y_{p}=\tilde{\boldsymbol{\theta}}^{T} \hat{\boldsymbol{\omega}}+\boldsymbol{\theta}^{T} \tilde{\boldsymbol{\omega}}
$$

then equation (3.33) becomes

$$
\dot{V}= \begin{cases}-\frac{1}{2} e^{2}-\frac{q}{2} \tilde{\boldsymbol{\theta}}^{T} \boldsymbol{\Gamma}^{-1} \tilde{\boldsymbol{\theta}} & \text { if }\|\boldsymbol{\Gamma}\| \leq r_{0} \\ -\frac{1}{2} e^{2}, & \text { otherwise }\end{cases}
$$

where $\Gamma$ is bounded and positive definite $\forall t \geq t_{0}$. Moreover, the state error $\dot{\tilde{\omega}}=\Lambda \tilde{\omega}$ converges to zero due to the choice of $\boldsymbol{\Lambda}$ and therefore, the Lyapunov derivative becomes negative semidefinite $\dot{V} \leq 0$. Hence, $\tilde{\theta}$ is bounded. Since this in turn implies that $\hat{\omega}$ is bounded as the plant is asymptotically bounded. If in addition, the input $u$ 
is smooth, we can show that $\dot{e}$ is bounded and hence using By Barbalat's Lemma it follows that $\lim _{t \rightarrow \infty} e(t)=0$. This implies that asymptotic state estimation is possible if $u$ is smooth. In order to achieve parameter estimation, the condition of persistent excitation

$$
\int_{t}^{t+T_{0}} \hat{\boldsymbol{\omega}} \hat{\boldsymbol{\omega}}^{T} \mathrm{~d} \tau \geq \alpha \mathbf{I} \quad \forall t \leq t_{0}
$$

where $t_{0}, T_{0}$ and $\alpha$ are positive constants, has to be satisfied.

\subsubsection{Extension of the nonminimal representation II for plants with direct feedtrough}

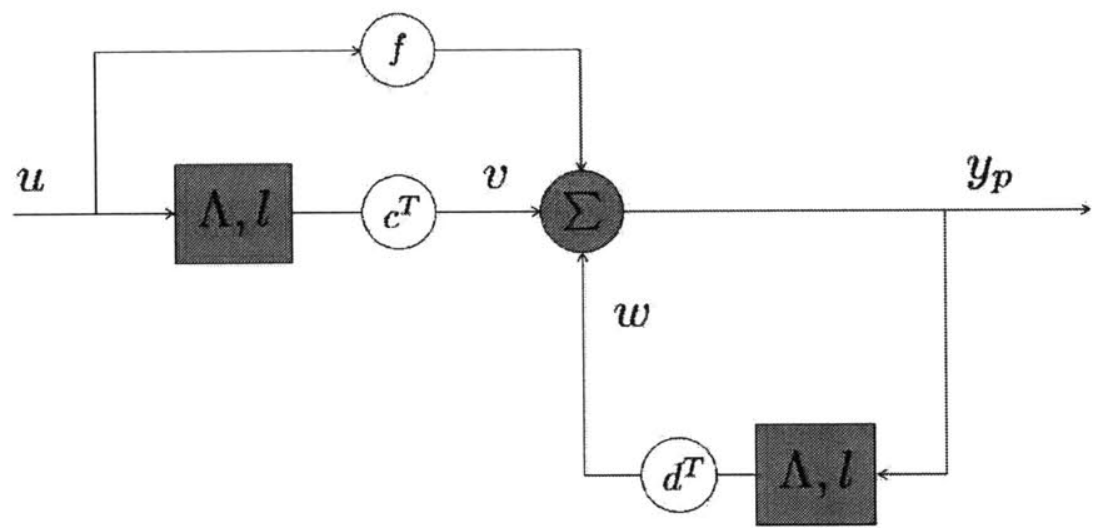

Figure 3-2: Nonminimal representation II

In the case, were the plant output has a direct feedthrough term

$$
y_{p}=\mathbf{c x}+d u
$$

the non-minimal representation can be extended as depicted in figure 3-2 by the term $f$

$$
\begin{aligned}
& \dot{\omega}_{1}=\Lambda \omega_{1}+\mathbf{l} u \\
& \dot{\omega}_{2}=\Lambda \omega_{2}+\mathbf{l} y_{p} \\
& y_{p}=\mathbf{c}^{T} \omega_{1}+\mathbf{d}^{T} \omega_{2}+f u .
\end{aligned}
$$


The transfer function for the nonminimal representation becomes in this case

$$
\frac{Y_{p}(s)}{U(s)}=\frac{P(s)+f R(s)}{R(s)-Q(s)}
$$

where $P(s), Q(s), R(s)$ defined as shown in equations (3.6) and (3.7). Input-output equivalence of the non-minimal plant

$$
W(s)=\frac{Y_{p}(s)}{U(s)}=\frac{f s^{n}+\left(f \Lambda_{n-1}+c_{n-1}\right) s^{n-1}+\ldots+\left(f \Lambda_{0}+c_{0}\right)}{s^{n}+\left(\Lambda_{n-1}-d_{n-1}\right) s^{n-1}+\ldots+\left(\Lambda_{0}-d_{0}\right)}
$$

with the plant transfer function

$$
W_{p}(s)=\boldsymbol{c}^{\boldsymbol{T}}(s \boldsymbol{I}-\boldsymbol{A})^{-1} \boldsymbol{b}+d=\frac{s^{n} b_{n}+s^{n-1} b_{n-1}+\ldots+s^{2} b_{2}+s b_{1}+b_{0}}{s^{n}+s^{n-1} a_{n-1}+s^{n-2} a_{n-2}+\ldots+s^{2} a_{2}+s a_{1}+a_{0}}
$$

can be achieved by setting

$$
\left[\begin{array}{c}
c_{0} \\
c_{1} \\
\vdots \\
c_{n-1} \\
f
\end{array}\right]=\left[\begin{array}{c}
b_{0}-b_{n} \Lambda_{0} \\
b_{1}-b_{n} \Lambda_{1} \\
\vdots \\
b_{n-1}-b_{n} \Lambda_{n-1} \\
b_{n}
\end{array}\right] \text { and }\left[\begin{array}{c}
d_{0} \\
\vdots \\
d_{n-1}
\end{array}\right]=\left[\begin{array}{c}
-a_{0}+\Lambda_{0} \\
\vdots \\
-a_{n-1}+\Lambda_{n-1}
\end{array}\right]
$$

It can be shown that the state transformation stays the same as in equation (3.13). 


\subsection{Adaptive observer for a single particle model plant}

In this section, we develop an adaptive observer based on the afore introduced nonminimal representation II and the single particle model. The single particle model is a simplification of the pseudo two dimensional model (P2D model), presented in the next chapter, for a coarsely discretized cell [2]. We show that based on the estimation

of states and specific parameters in the single particle model, the state of charge of each electrode, the available cell power and capacity of a li-ion intercalation cell can be inferred. Moreover, we discuss intricacies of the single particle model, which include weak observability due to a large feed-through signal and separated timescales. In the last section, we validate the observer on a linear and a nonlinear single particle plant model.

\subsubsection{The Single Particle Model}

In this section, we derive the single particle model from the P2D model. For a longer introduction to the P2D model, the reader is referred to section 4.1. Notations are used as shown in table A. The single particle model discretizes the cell by defining one node for each electrode and for the separator. States in each compartment are then approximated by their averaged values in $\mathrm{x}$-direction. Hence, each electrode is approximated as one single particle with equivalent volume and area. In addition, the electrolyte concentration is assumed to be constant throughout the cell. This assumption is valid for cells with high electrolyte conductivity $\kappa$ or when applied cell currents are very low $I(t) \ll 1 C$. Thus, the spatial derivative of the electrolyte concentration $\frac{\partial c_{e}}{\partial x}$ vanishes and equation 4.11 can be regarded as unmodeled dynamics. Spatial variations in the pseudo direction $r$ remain.

The surface flux of the electrode has no x-variation $j(x, t)=j(t)$ due to eq.(4.11). 
By integration of eq.(4.2)

$$
\int_{0}^{L^{ \pm}} \frac{\partial i_{e}}{\partial x} \mathrm{dx}=\int_{0}^{L^{ \pm}} F a^{ \pm} j^{ \pm}(t) d x=F a^{ \pm} j^{ \pm}(t) L^{ \pm}
$$

and using boundary conditions

$$
\int_{0}^{L^{ \pm}} \frac{\partial i_{e}}{\partial x} \mathrm{dx}=i_{e}\left(L^{ \pm}\right)-i_{e}(0)=\mp I
$$

we obtain a closed solution for the flux

$$
j^{ \pm}(t)=\mp \frac{I}{F a^{ \pm} L^{ \pm}}
$$

The solid potential is approximated by the node values for each electrode

$$
\phi_{s}(x, t)= \begin{cases}\phi_{s}^{+}(t) & \text { for } 0^{+} \leq x \leq L^{+} \\ \phi_{s}^{-}(t) & \text { for } 0^{-} \leq x \leq L^{-}\end{cases}
$$

Due to the assumption $\frac{I(t)}{\kappa} \ll 1$ and $\left|i_{e}(t)\right| \leq|I(t)|$ we obtain from eq.(4.7)

$$
\begin{aligned}
\phi_{e}(x, t) & =\phi_{e}\left(0^{-}, t\right)+\int_{0^{-}}^{x} \frac{\partial \phi_{e}(x, t)}{\partial x} \mathrm{dx} \\
& =\phi_{e}\left(0^{-}, t\right)+\int_{0^{-}}^{x} \frac{i_{e}(x, t)}{\kappa} \mathrm{dx} \approx \phi_{e}\left(0^{-}, t\right) .
\end{aligned}
$$

Given the boundary condition eq.(4.10), the electrolyte potential within the cell is

$$
\phi_{e}(t)=0
$$

The solid concentration on each electrode side is described by the diffusion in the pseudo sphere. The PDEs describing the dynamics are

$$
\frac{\partial c_{s}^{ \pm}(r, t)}{\partial t}=\frac{1}{r^{2}} \frac{\partial}{\partial r}\left(D_{s}^{ \pm} r^{2} \frac{\partial c_{s}^{ \pm}(r, t)}{\partial r}\right)
$$


with boundary conditions

$$
\begin{aligned}
\left.\frac{\partial c_{s}^{ \pm}}{\partial r}\right|_{r=R_{p}^{ \pm}} & =\frac{I(t)}{D_{s}^{ \pm} F a^{ \pm} L^{ \pm}} \\
\left.\frac{\partial c_{s}^{ \pm}}{\partial r}\right|_{r=0} & =0
\end{aligned}
$$

and initial conditions

$$
c_{s}^{-}(r, 0)=c_{s}^{-, 0}(r)
$$

Finally, the Butler Volmer equations (4.23) can be expressed as

$$
\mp \frac{I(t)}{F a^{ \pm} L^{ \pm}}=i_{0}^{ \pm}(t)\left(\exp \left(\frac{\alpha_{a} F}{R T} \eta^{ \pm}\right)-\exp \left(-\frac{\alpha_{c} F}{R T} \eta^{ \pm}\right)\right)
$$

where

$$
i_{0}^{ \pm}(t)= \begin{cases}k^{i} c_{e}^{\alpha_{a}}\left(c_{s, \text { max }}^{+}-c_{s s}^{+}(t)\right)^{\alpha_{a}} c_{s s}^{+}(t)^{\alpha_{c}} & \text { for } 0^{+} \leq x \leq L^{+} \\ k^{i} c_{e}^{\alpha_{a}}\left(c_{s, \text { max }}^{-}-c_{s s}^{-}(t)\right)^{\alpha_{a}} c_{s s}^{-}(t)^{\alpha_{c}} & \text { for } 0^{-} \leq x \leq L^{-}\end{cases}
$$

and

$$
\eta(x, t)= \begin{cases}\phi_{s}^{+}(t)-\mathcal{U}^{+}\left(c_{s s}^{+}(t)\right)+R_{S E I}^{+} \frac{I}{a^{+} L^{+}} & \text {for } 0^{+} \leq x \leq L^{+} \\ \phi_{s}^{-}(t)-\mathcal{U}^{-}\left(c_{s s}^{-}(t)\right)-R_{S E I}^{-} \frac{I}{a^{-} L^{-}} & \text {for } 0^{-} \leq x \leq L^{-}\end{cases}
$$

In the symmetric case, where $\alpha_{a}=\alpha_{c}=0.5$, we can obtain a direct solution of the cell voltage as

$$
\begin{aligned}
V(t)=\phi_{s}^{+}(t)-\phi_{s}^{-}(t) & =\mathcal{U}^{+}\left(c_{s s}^{+}(t)\right)-\mathcal{U}^{-}\left(c_{s s}^{-}(t)\right) \\
& +\frac{2 R T}{F}\left(\sinh ^{-1}\left(\frac{I(t)}{F a^{+} L^{+} i_{0}^{+}(t)}\right)-\sinh ^{-1}\left(\frac{I(t)}{F a^{-} L^{-} i_{0}^{-}(t)}\right)\right) \\
& -R_{S E I}^{+} \frac{I}{a^{+} L^{+}}-R_{S E I}^{-} \frac{I}{a^{-} L^{-}}
\end{aligned}
$$

This nonlinear cell voltage equation and the two PDE equations (3.49) describe the li-ion cell. 


\subsubsection{Simplified SPM: Volume averaged projections and lin- earized cell voltage}

In this subsection, we derive a simplified single particle model by applying volumeaveraging projections on the solid diffusion PDEs and by linearizing the cell voltage function. We apply volume-averaging projections, which will be introduced in detail in section 4.2 .4 , on equations (3.49), to obtain

$$
\begin{aligned}
\frac{\partial}{\partial t} \bar{c}_{s}^{i}(t) & =-3 \frac{j^{i}(t)}{R_{p}^{i}} \\
\frac{\partial}{\partial t} \bar{q}^{i}(t) & =-30 \frac{D_{s}^{i}}{\left(R_{p}^{i}\right)^{2}} \bar{q}(t)-\frac{45}{\left(R_{p}^{i}\right)^{2}} j^{i}(t)
\end{aligned}
$$

where $i= \pm, \bar{c}_{s}^{i}(t)$ represents the volume-averaged solid concentration and $\bar{q}^{i}(t)$ is defined as the volume-averaged solid fluxes. The volume-averaging projections have been validated by [21] [3] to precisely approximate the PDEs up to $1 C$ discharge and charge rate. If side reactions and loss of active material are neglected, then the conservation of recyclable lithium is defined by

$$
\frac{4}{3} \pi\left(R^{-}\right)^{3} \bar{c}_{s}^{-}+\frac{4}{3} \pi\left(R^{+}\right)^{3} \bar{c}_{s}^{-}=m_{L i}
$$

where parameter $m_{L i}$ denotes the total Lithium mass in the cell. Therefore, $\bar{c}_{s}^{-}$and $\bar{c}_{s}^{-}$are dependent and only one is necessary for a minimal plant description. The state-space representation is then defined by

$$
\dot{\mathbf{x}}=\mathbf{A x}+\mathbf{b} u
$$

where

$$
\mathbf{x}=\left[\begin{array}{c}
\bar{c}_{s}^{-}(t) \\
\bar{q}^{-}(t) \\
\bar{q}^{+}(t)
\end{array}\right], \quad u=I(t)
$$


and

$$
\mathbf{A}=\left[\begin{array}{ccc}
0 & 0 & 0 \\
0 & -30 \frac{D_{s}^{-}}{\left(R_{p}^{-}\right)^{2}} & 0 \\
0 & 0 & -30 \frac{D_{s}^{+}}{\left(R_{p}^{+}\right)^{2}}
\end{array}\right], \quad \mathbf{b}=\left[\begin{array}{c}
-\frac{3}{F a^{-} L^{-} R_{p}^{-}} \\
-\frac{45}{\left(R_{p}^{-}\right)^{2} F a^{-} L^{-}} \\
\frac{45}{\left(R_{p}^{+}\right)^{2} F a^{+} L^{+}}
\end{array}\right]
$$

To further simplify the model, we can linearly approximate the cell voltage at a certain operating point $\left(\mathrm{x}_{0}, u_{0}\right)$ by

$$
V(x, t) \approx V\left(\mathbf{x}_{0}, u_{0}\right)+\left.\frac{\partial V}{\partial \mathbf{x}}\right|_{\mathbf{x}=\mathbf{x}_{0}, u=u_{0}} \Delta \mathbf{x}+\left.\frac{\partial V}{\partial u}\right|_{\mathbf{x}=\mathbf{x}_{0}, u=u_{0}} \Delta u
$$

where $\mathbf{x}=\mathbf{x}_{0}+\Delta \mathbf{x}$ and $u=u_{0}+\Delta u$. If we choose the operating point to be a cell at chemical equilibrium, i.e. there are no fluxes in the cell, then

$$
\mathbf{x}_{0}=\left[\begin{array}{c}
\overline{c_{s, 0}} \\
0 \\
0
\end{array}\right] \text { and } u_{0}=0
$$

The cell voltage for small deviations from this equilibrium point is

$$
\begin{aligned}
& V(\mathbf{x}, t)=V\left(\mathbf{x}_{0}, u_{0}\right)+\left[-\left.\frac{\partial U^{-}}{\partial c_{s s}^{-}}\right|_{c_{s s}^{-}=c_{s, i n i t}^{-}}-\left.\frac{\partial U^{+}}{\partial c_{s s}^{+}}\right|_{c_{s s}^{+}=c_{s, i n i t}^{+}}\left(\frac{R_{p}^{-}}{R_{p}^{+}}\right)^{3}\right] \Delta x_{1}+ \\
& {\left[-\left.\frac{\partial U^{-}}{\partial c_{s s}^{-}}\right|_{c_{s s}^{-}=c_{s, \text { init }}^{-}} \frac{8}{35} R_{p}^{-}\right] \Delta x_{2}+\left[\left.\frac{\partial U^{+}}{\partial c_{s s}^{+}}\right|_{c_{s s}^{+}=c_{s, \text { init }}^{+}} \frac{8}{35} R_{p}^{+}\right] \Delta x_{3}+} \\
& {\left[-\frac{R T}{F^{2} a^{+} L^{+} r_{e f f}^{+} \sqrt{c_{e}^{0} c_{s s}^{+}\left(c_{s, \text { max }}^{+}-c_{s s}^{+}\right)}}-\frac{R T}{F^{2} a^{-} L^{-} r_{e f f}^{-} \sqrt{c_{e}^{0} c_{s s}^{-}\left(c_{s, \text { max }}^{-}-c_{s s}^{-}\right)}}\right.} \\
& -\frac{R_{f}^{-}}{a^{-} L^{-}}-\frac{R_{f}^{+}}{a^{+} L^{+}}+\left.\frac{\partial U^{+}}{\partial c_{s s}^{+}}\right|_{c_{s s}^{+}=c_{s, \text { init }}^{+}}\left(\frac{R_{p}^{+}}{35 D_{s}^{+} F a^{+} L^{+}}\right) \\
& \left.+\left.\frac{\partial U^{-}}{\partial c_{s s}^{-}}\right|_{\overline{s s}=c_{s, \text { init }}^{-}}\left(\frac{R_{p}^{-}}{35 D_{s}^{-} F a^{-} L^{-}}\right)\right] \Delta u
\end{aligned}
$$


We now define our output as $y=V(\mathbf{x}, t)-V\left(\mathbf{x}_{0}, u_{0}\right)$ which results in the state space description

$$
y=\mathbf{c}^{T} \Delta \mathbf{x}+d \Delta u
$$

where

$$
\mathbf{c}=\left[\begin{array}{c}
-\left.\frac{\partial U^{-}}{\partial c_{s s}^{-}}\right|_{c_{s s}^{-}=c_{s, i n i t}^{-}}-\left.\frac{\partial U^{+}}{\partial c_{s s}^{+}}\right|_{c_{s s}^{+}=c_{s, i n i t}^{+}}\left(\frac{R_{p}^{-}}{R_{p}^{+}}\right)^{3} \\
-\left.\frac{\partial U^{-}}{\partial c_{s s}^{-}}\right|_{c_{s s}^{-}=c_{s, i n i t}^{-}} \frac{8}{35} R_{p}^{-} \\
\left.\frac{\partial U^{+}}{\partial c_{s s}^{+}}\right|_{c_{s s}^{+}=c_{s, i n i t}^{+}} \frac{8}{35} R_{p}^{+}
\end{array}\right]
$$

and

$$
\begin{aligned}
& d=-\frac{R T}{F^{2} a^{+} L^{+} r_{e f f}^{+} \sqrt{c_{e}^{0} c_{s s}^{+}\left(c_{s, \text { max }}^{+}-c_{s s}^{+}\right)}}-\frac{R T}{F^{2} a^{-} L^{-} r_{e f f}^{-} \sqrt{c_{e}^{0} c_{s s}^{-}\left(c_{s, \text { max }}^{-}-c_{s s}^{-}\right)}} \\
& -\frac{R_{f}^{-}}{a^{-} L^{-}}-\frac{R_{f}^{+}}{a^{+} L^{+}}+\left.\frac{\partial U^{+}}{\partial c_{s s}^{+}}\right|_{c_{s s}^{+}=c_{s, \text { init }}^{+}}\left(\frac{R_{p}^{+}}{35 D_{s}^{+} F a^{+} L^{+}}\right)+\left.\frac{\partial U^{-}}{\partial c_{s s}^{-}}\right|_{c_{s s}^{-}=c_{s, \text { init }}^{-}}\left(\frac{R_{p}^{-}}{35 D D_{s}^{-} F a^{-} L^{-}}\right)
\end{aligned}
$$

\subsubsection{States and parameters of interest}

Based on estimates of states (3.61) and specific parameters of the single particle model, we can apply further transformations, which provide us the variables of interest for the battery monitoring system. Bulk SOC for each electrode is defined as

$$
S O C^{ \pm}=\frac{\bar{c}_{s}^{ \pm}}{c_{s, \text { max }}^{ \pm}}
$$

where $c_{s, \text { max }}^{ \pm}$is defined as the theoretical nominal concentration of lithium in the electrode. Available power is directly proportional to the surface SOC

$$
c_{s s}(t)^{ \pm}= \pm \frac{1}{35} \frac{I R_{p}^{ \pm}}{F D_{s}^{ \pm} a^{ \pm} L^{ \pm}}+\frac{8}{35} R_{p}^{i} \bar{q}(x, t)+\bar{c}(x, t)
$$

through the OCV of the cell [2] and pulse power depends on $c_{s s}(t)$ and $D_{s}^{ \pm}$. By $D_{s}^{ \pm}$ predictions of $c_{s s}(t)$ for a time horizon of $2 s-10 s$ are possible, e.g. by pre-simulating the solid diffusion in open-loop for the given prediction horizon. Finally, capacity of 
the cell can be derived by knowing the volume of the active material defined by the radius $R_{p}^{ \pm}$of the pseudo sphere and the amount of total recyclable lithium $m_{L i}$ in the cell. Capacity $C_{c a p}$ is then defined by as

$$
C_{c a p}=\left\{\begin{aligned}
\frac{3}{4} \pi\left(R_{p}^{+}\right)^{3} c_{s, \text { max }}^{+} & \text {if }\left(R_{p}^{+}\right)^{3} c_{s, \text { max }}^{+}<\left(R_{p}^{-}\right)^{3} c_{s, \text { max }}^{-} \text {and } \frac{3}{4} \pi\left(R_{p}^{+}\right)^{3} c_{s, \text { max }}^{+}<m_{L i} \\
\frac{3}{4} \pi\left(R_{p}^{-}\right)^{3} c_{s, \text { max }}^{-} & \text {if }\left(R_{p}^{-}\right)^{3} c_{s, \text { max }}^{-}<\left(R_{p}^{+}\right)^{3} c_{s, \text { max }}^{+} \text {and } \frac{3}{4} \pi\left(R_{p}^{-}\right)^{3} c_{s, \text { max }}^{-}<m_{L i} \\
m_{L i} & \text { if } m_{L i}<\frac{3}{4} \pi\left(R_{p}^{+}\right)^{3} c_{s, \text { max }}^{+} \text {and } m_{L i}<\frac{3}{4} \pi\left(R_{p}^{-}\right)^{3} c_{s, \text { max }}^{-}
\end{aligned}\right.
$$

Hence, capacity is always equal to the smallest and therefore limiting electrode capacity or equal to the total lithium content, if none of the electrode can be fully charged. As mentioned in chapter 2.2 capacity and internal impedances, such as the solid diffusion, alter over time due to aging. We are therefore have to track these parameters

$$
\theta=\left[\begin{array}{lllll}
R_{p}^{+} & R_{p}^{-} & m_{L i} & D_{s}^{+} & D_{s}^{-}
\end{array}\right]
$$

with the adaptive observer.

\subsubsection{Observability and stability of the SPM}

The observability of the single particle model is a requirement for the adaptive observer presented in the last section. However, a quick check on different cell chemistries reveals that the observability matrix

$$
\mathbb{O}=\left[\begin{array}{c}
c \\
c A \\
\vdots \\
c A^{n-1}
\end{array}\right]
$$

is badly conditioned over a wide range of operating points. We investigated this feature in depth for a $\mathrm{LiCoO}_{2} / \mathrm{LiC}_{6}$ cell by evaluating the observability over the whole SOC range of the cell. Numerical calculations revealed that $\mathbb{O}$ was better conditioned towards low SOC values $(S O C<0.2)$. This behavior was also found 
for a $\mathrm{LiMnO}_{2} / \mathrm{LiC}_{6}$ cell. It can be further shown that improving condition numbers of the observability matrix coincide with increasing downward sloping of the OCV curves. This phenomenon can be best understood by analyzing the pole-zero locations of the plant and the OCV curve properties. Let us define the plant transfer function of the SPM by

$$
W_{p}(s)=\frac{Z(s)}{P(s)}+d
$$

where

$$
\frac{Z(s)}{P(s)}=\mathbf{c}^{\mathbf{T}}(s \mathbf{I}-\mathbf{A})^{-1} \mathbf{b}=\frac{z_{2} s^{2}+z_{1} s+z_{0}}{s^{3}+p_{2} s^{2}+p_{1} s^{3}+p_{0}}
$$

with

$$
\left[\begin{array}{l}
p_{0} \\
p_{1} \\
p_{2}
\end{array}\right]=\left[\begin{array}{c}
0 \\
30\left(\frac{D_{s}^{+}}{\left(R_{p}^{+}\right)^{2}}+\frac{D_{s}^{-}}{\left(R_{p}^{-}\right)^{2}}\right) \\
900 \frac{D_{s}^{+} D_{s}^{-}}{\left(R_{p}^{+}\right)^{2}\left(R_{p}^{-}\right)^{2}}
\end{array}\right] \text { and }\left[\begin{array}{c}
z_{0} \\
z_{1} \\
z_{2}
\end{array}\right]=[a]
$$

By using the same proof of the root-locus analysis, it becomes obvious that for $\frac{z_{2}}{p_{2}}, \frac{z_{1}}{p_{1}}, \frac{z_{0}}{p_{0}}<<d$ zeros of $W_{p}(s)$ converge towards the poles of the system. This condition is fulfilled for low values of $\frac{\partial \mathcal{U}^{+}}{\partial c_{s s}^{+}}$and $\frac{\partial \mathcal{U}^{-}}{\partial c_{s s}^{-}}$, i.e. where OCV curves form a plateau like profile. Thus, at low SOC values, where the OCV gradient drastically increases, the system becomes strongly observable. The single particle model is a marginally stable plant, i.e. we have a pole at zero

$$
\psi_{1}=0
$$

and two poles in the left-half plane

$$
\psi_{2}=-\frac{30 D_{s}^{+}}{\left(R_{p}^{+}\right)^{2}}, \psi_{3}=-\frac{30 D_{s}^{-}}{\left(R_{p}^{-}\right)^{2}} .
$$

However, we know that signals of the plant are all bound due to the inherent cell physics. The cell voltage will be always kept between two cutoff voltages and therefore, the stability proof for the adaptive observer in chapter 3.1 still holds. For certain cell chemistries, the two poles $\psi_{2}$ and $\psi_{3}$ are of different order of magnitude. The dynamics of the volume-averaged fluxes $\bar{q}^{+}(t)$ and $\bar{q}^{-}(t)$ have therefore time constants 
of different order of magnitude. This wide separation of dynamics can obstruct parameter estimation, due to the faster pole dominating the output signal.

\subsubsection{Simulation setup and results}

To estimate parameters of a $\mathrm{LiCoO}_{2} / \mathrm{LiC}_{6}$ cell, we use the adaptive observer with a feed-through term as shown in section 3.1.2 combined with the recursive least square algorithm for parameter updates.

We analyze the observer performance for two cases. First, we apply the observer on the simplified SPM as described in chapter 3.2.2. For the second case, we simulate the observer on the nonlinear SPM presented in 3.2.1 for low amplitude input current shown in 3-18 and then for large amplitude input current shown in 3-25. We choose initial states of the plant to be $S O C_{a}=0.8$ and $S O C_{c}=0.28$. The cell starts from equilibrium and is subjected to a superposition of harmonic inputs, which creates a persistently exciting signal. Frequencies of the superposed sinuses for the first case are shown in A.2 and for the second case in A.3. Parameter values of the real plant are chosen as shown in table A.2. Initial conditions of the estimated parameters are chosen to be off by $60 \%$. The initial estimate of the cell states have a $10 \%$ error. Filter values of the observer are chosen to be near the initial estimates for plant poles.

They are $\lambda_{1}=10^{-2}, \lambda_{2}=10^{-3}$ and $\lambda_{3}=10^{-4}$. The optimal choice of filter values is a topic of on-going research. The initial gain for the recursive least square algorithm and the forgetting factor are varied according to the input current magnitude. The simulations results are validated by comparing input output behavior in Bode diagrams, pole and zero locations and analyzing parameter transients.

For the first case, the input current reaches maximum peaks of $1 C$ magnitude $(1 C=$ $\left.6 \mathrm{~A} / \mathrm{m}^{2}\right)$ 3-10. Parameter estimates over time for the simulation of the adaptive observer on the linear SPM plant are shown in figure 3-3 and 3-4. All parameters have been normalized to a value of 1 . They converge as expected and usually exhibit transients of $200 s$. 
Parameter estimates of $c$
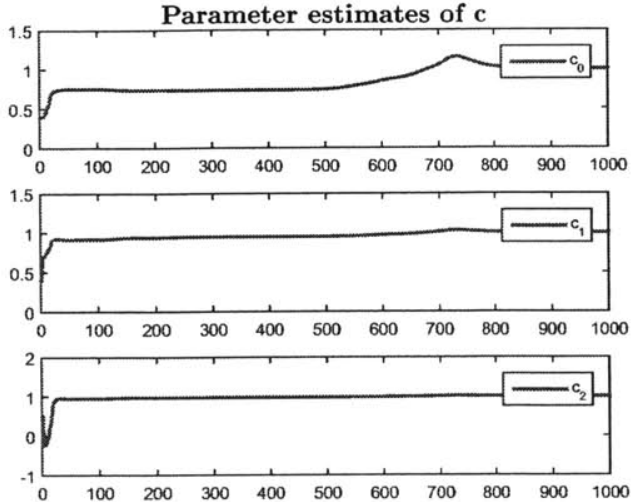

Figure 3-3: Parameter estimates for $t=$ $0-1000 s$. All parameters estimates have been normalized by their actual parameter value.
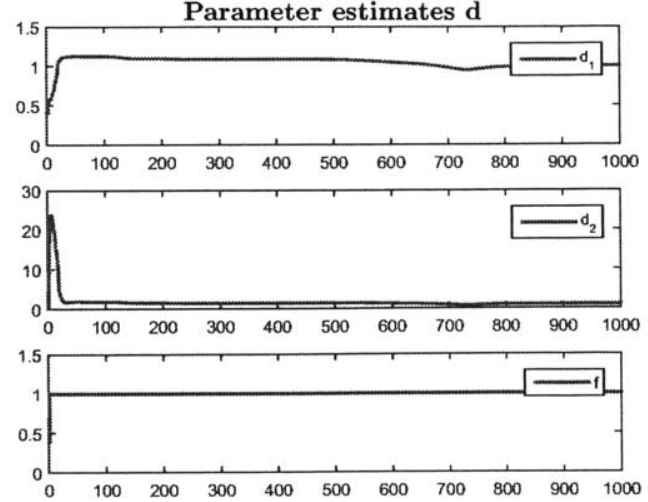

Figure 3-4: Parameter estimates for $t=$ $0-1000 s$. All parameters estimates have been normalized by their actual parameter value.

A comparison of the Bode diagrams and pole and zero locations after $1000 \mathrm{~s}$ shows perfect matching of the estimated plant with the actual plant.

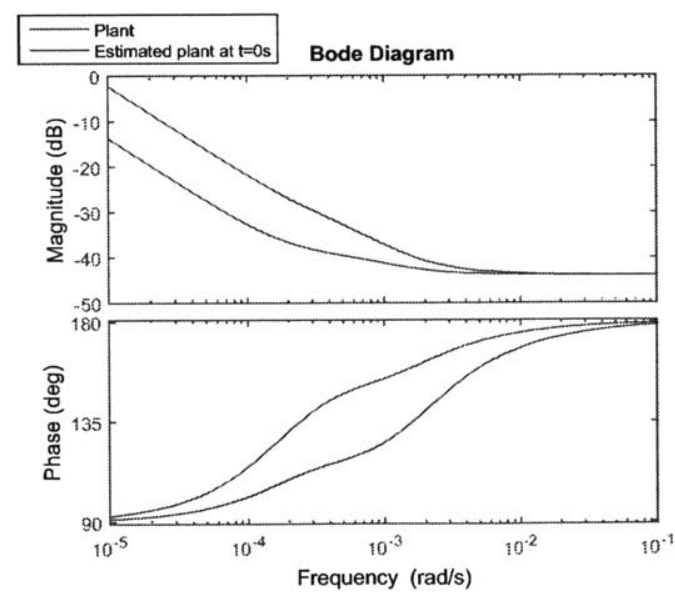

Figure 3-5: Bode diagram for initial plant estimation and linear plant.

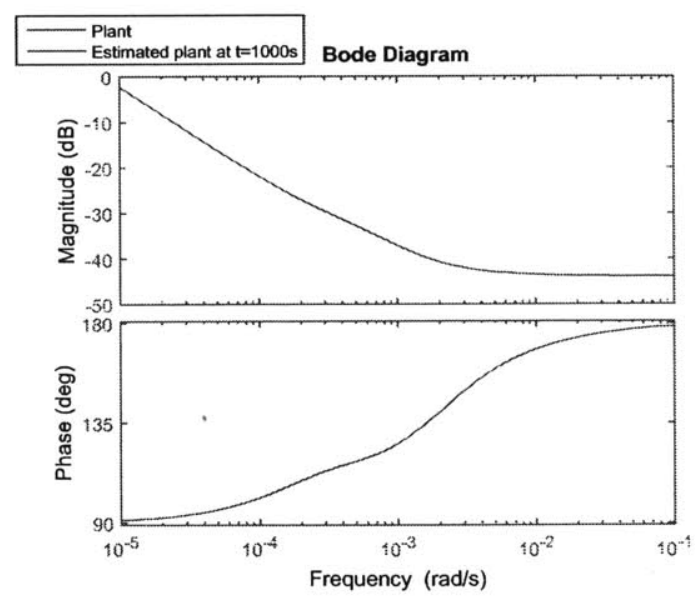

Figure 3-6: Bode diagram for estimated plant after $1000 \mathrm{~s}$ and linear plant. 


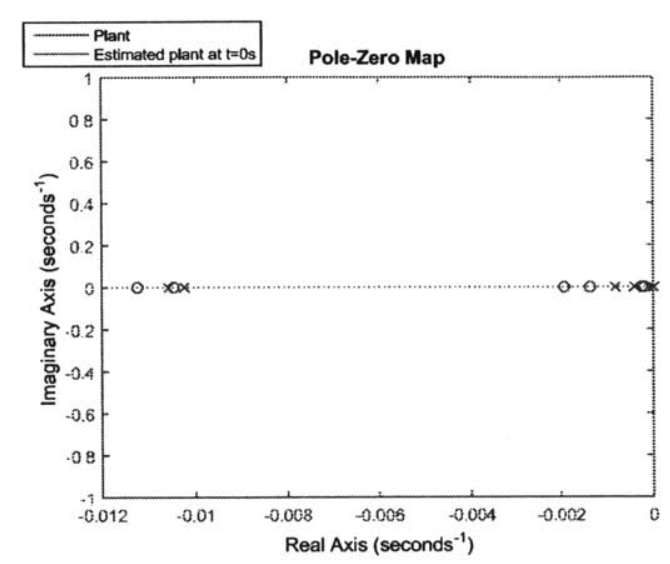

Figure 3-7: Pole and zero locations for initial plant estimate and linear plant.

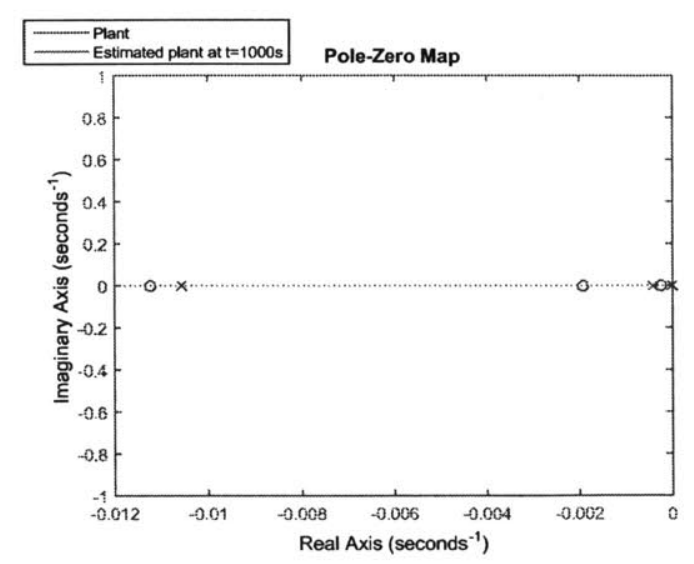

Figure 3-8: Pole and zero locations for estimated plant after $1000 \mathrm{~s}$ and linear plant.

As predicted by the stability analysis, the observer is globally stable if applied to the linear plant. The output error as defined in (3.34) converges to zero as shown in 3-9.

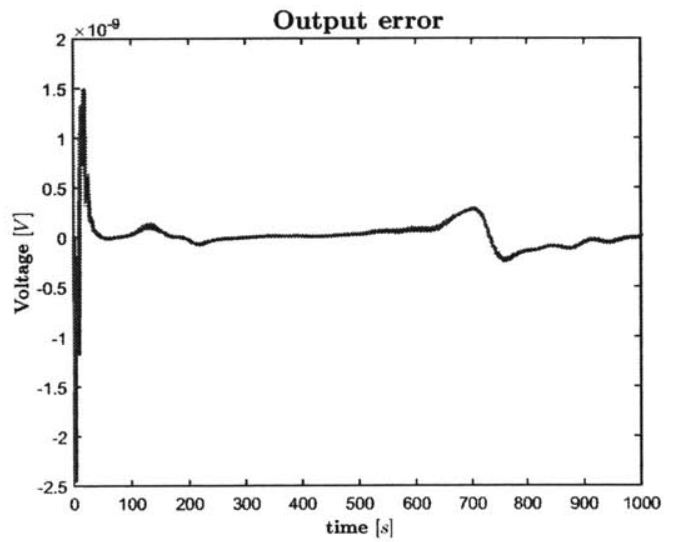

Figure 3-9: Error between plant output and estimated output.

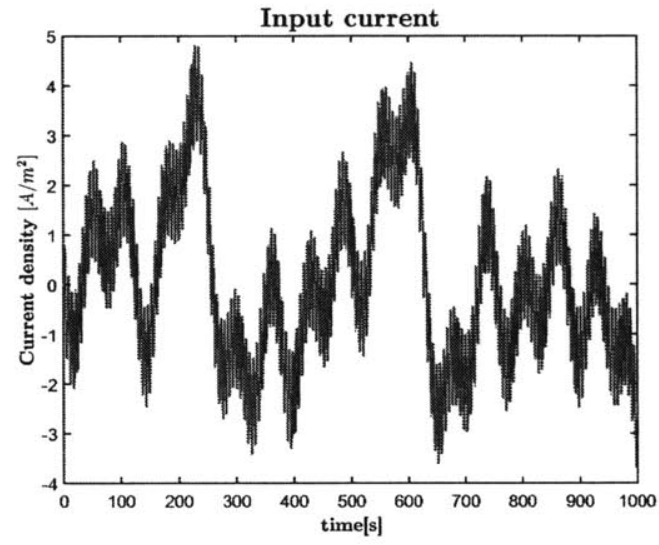

Figure 3-10: Current input to the linear plant.

The same observer design for the linear SPM plant is now simulated on the full SPM plant described in section 3.2.1. We first show a test case with small current inputs with current peaks of at most $0.1 C$, where nonlinearities due to Butler-Volmer kinetics and the OCV curves are still weak and the linear approximation holds. The parameter transients are shown in 3-11 and 3-12. 



Figure 3-11: Parameter estimates for $t=$ $0-1000 s$. All parameters have been normalized to value 1 .
Parameter estimates of $d$
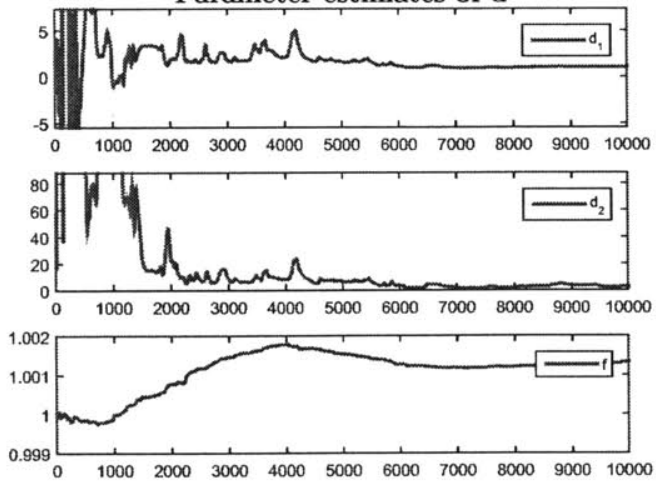

Figure 3-12: Parameter estimates for $t=$ $0-1000 s$. All parameters have been normalized to value 1 .

Performance on the nonlinear plant considerably decreases, but parameters satisfactorily converge after $t=10000 \mathrm{~s}$. Although nonlinearities are present, the recursive least square algorithm averages them out and parameter estimates converge to create an input output behavior equivalent to the nonlinear plant. This can observed by comparing Bode diagrams of the estimated linear plant and the single particle model plant linearized for initial conditions at $t=0 \mathrm{~s}$ in figure 3-13 and after $t=10000 \mathrm{~s}$ in figure 3-14. Pole and zero locations of the estimated plant are within 5\% error boundaries.

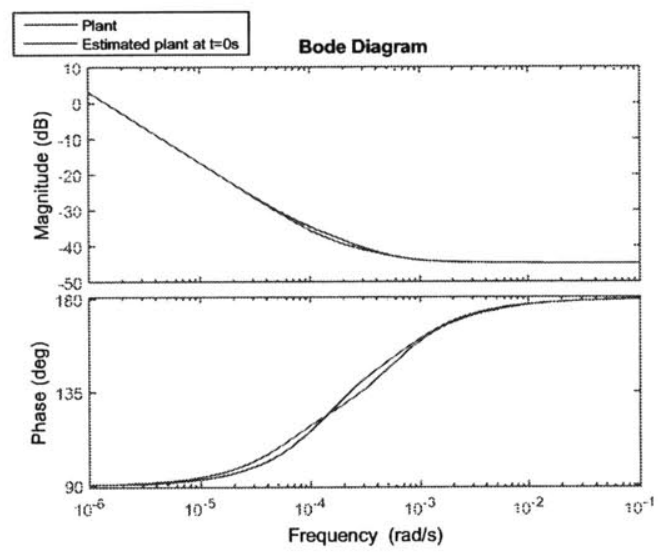

Figure 3-13: Bode diagram for initial plant estimation and nonlinear plant.

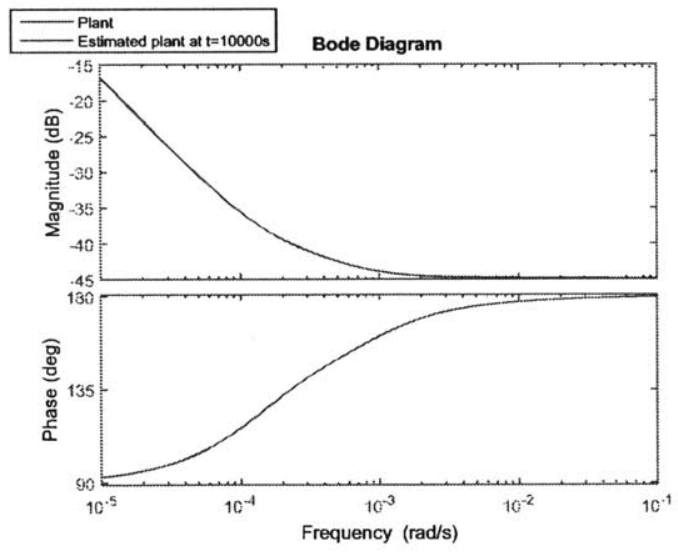

Figure 3-14: Bode diagram for estimated plant after $10000 s$ and nonlinear plant. 


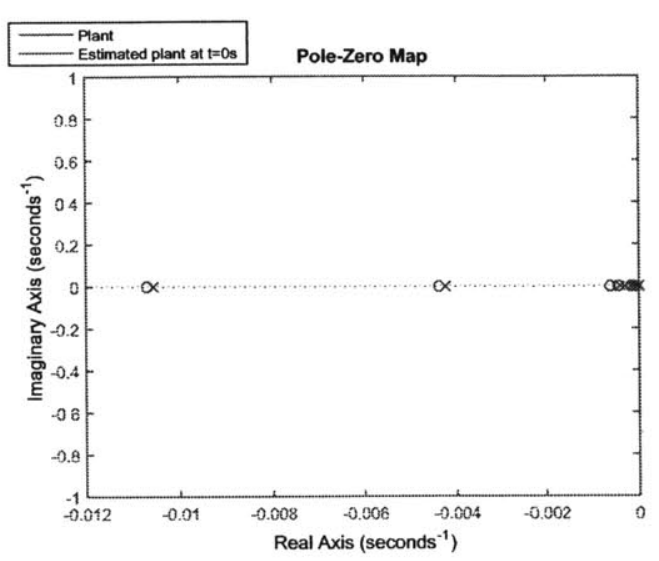

Figure 3-15: Pole and zero locations for initial plant estimate and nonlinear plant.

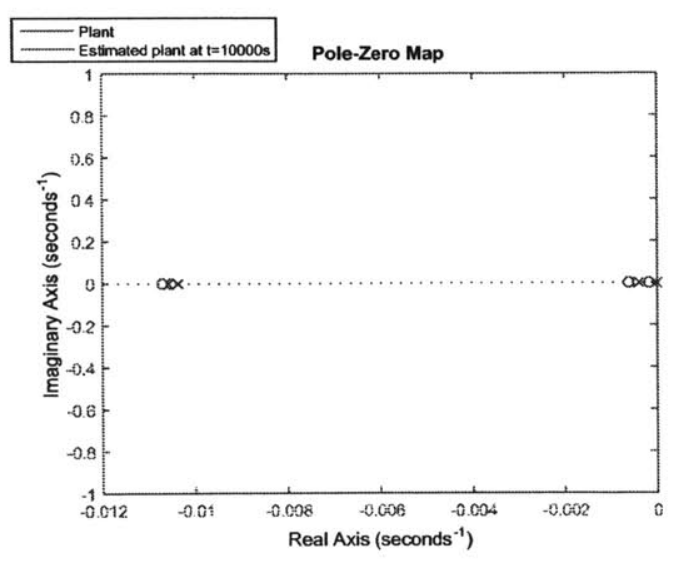

Figure 3-16: Pole and zero locations for estimated plant after $10000 \mathrm{~s}$ and nonlinear plant.

Unlike in the linear case, the error between the plant and the observer output does not converge to zero as shown in 3-17.

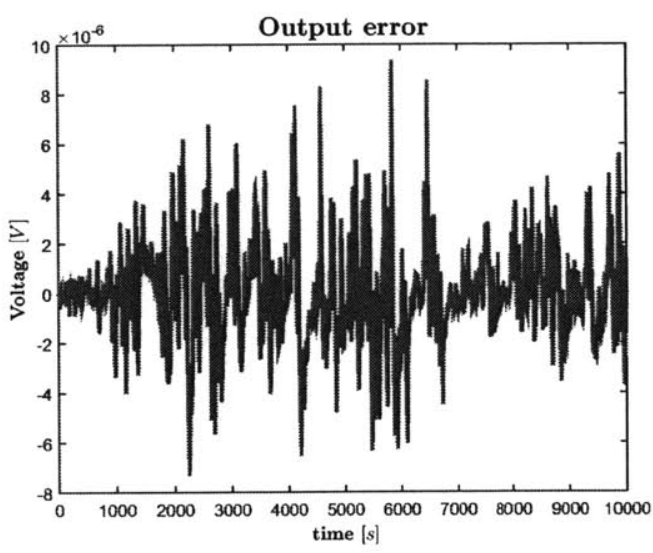

Figure 3-17: Error between plant output and estimated output.

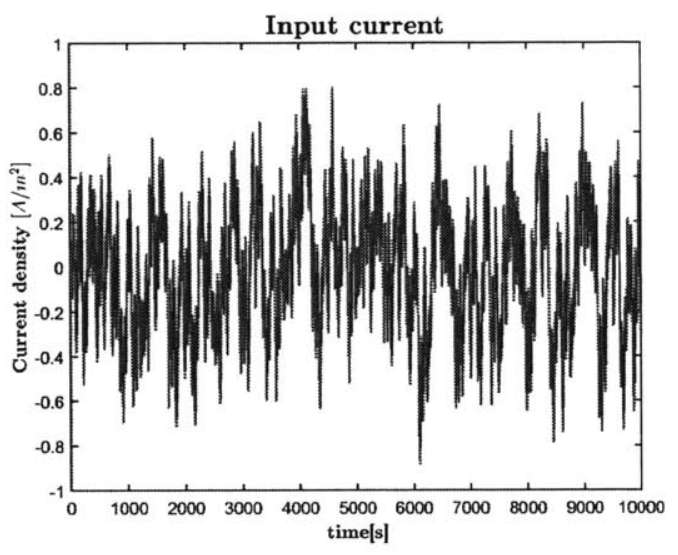

Figure 3-18: Current input to the linear plant.

In the last case, we simulate the observer on the nonlinear single particle model for higher currents with maximum peaks of $1 C$. We increase the forgetting factor of the recursive least square algorithm to $\beta=10^{-3}$, in order to estimate the poles despite the nonlinearities. By increasing the forgetting factor, the recursive least square algorithm retains information for shorter time and hence, shorter output ranges. Therefore, dynamics of states are less distorted by the nonlinear mapping to 
the output. Parameter estimates for the last case are shown in 3-19 and 3-20. The adaptive observer does not completely converge, due to the strong nonlinearities in the output function of the SPM 3-20.
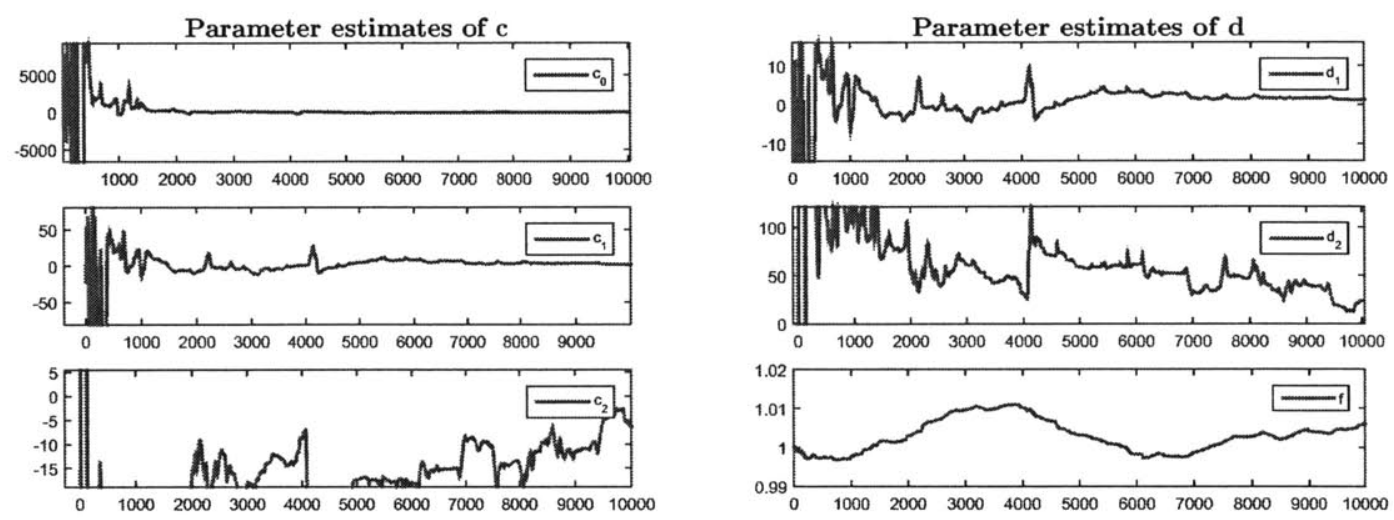

Figure 3-19: Parameter estimates for $t=$ $0-1000 s$. All parameters have been normalized to value 1 .

Figure 3-20: Parameter estimates for $t=$ $0-1000 s$. All parameters have been normalized to value 1 .

Both, the Butler Volmer kinetics and the change in open circuit potential become non-negligible and contribute significantly to the output of the plant. For plants with flat OCV profiles, the Butler-Volmer kinetics are the primary nonlinearities. Therefore, the transfer functions of the estimated plant and of the linearized plant doe not coincide as shown by the Bode plots in 3-21 and 3-22.

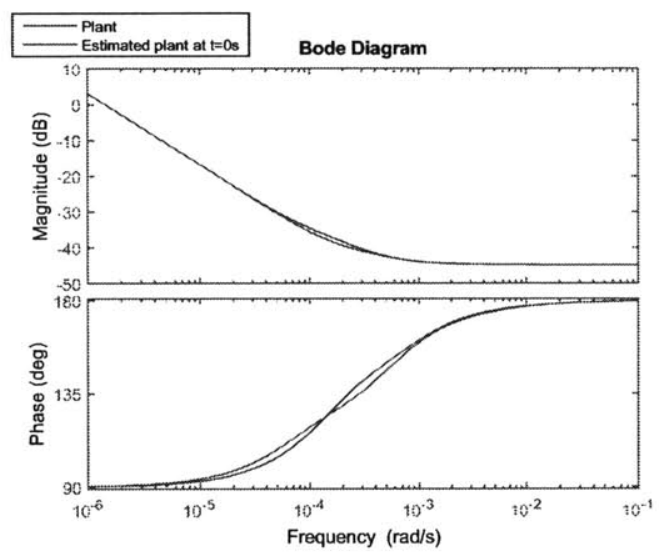

Figure 3-21: Bode diagram for initial plant estimation and nonlinear plant.

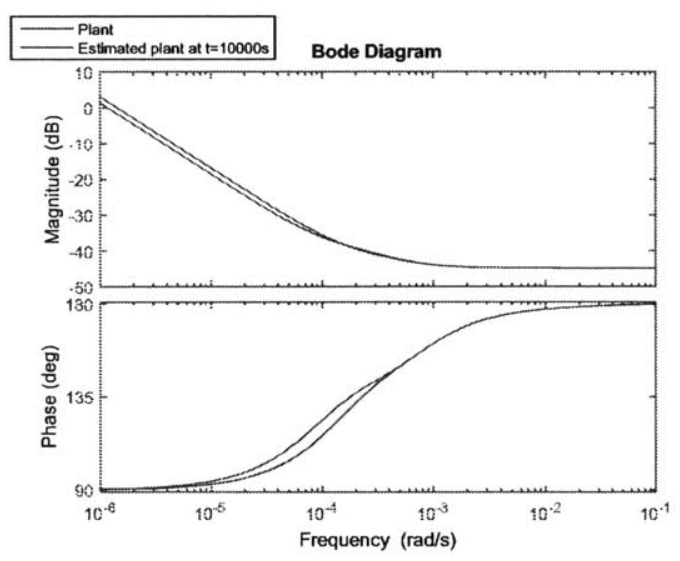

Figure 3-22: Bode diagram for estimated plant after $10000 \mathrm{~s}$ and nonlinear plant. 
Large poles in particular are estimated less precisely, because of the fast die off of related dynamics. This can be seen by comparing the pole zero locations in $3-23$ and 3-24.

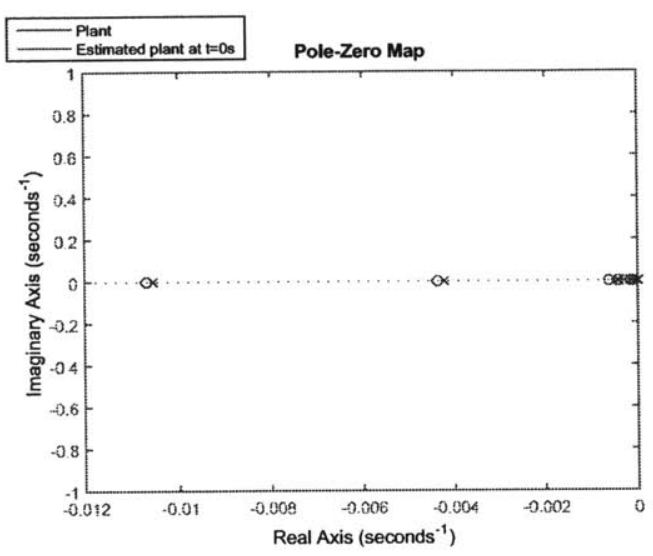

Figure 3-23: Pole and zero locations for initial plant estimate and nonlinear plant.

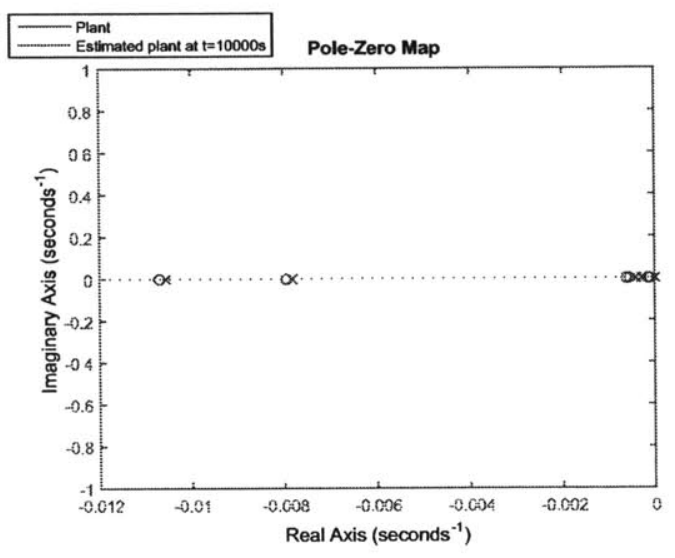

Figure 3-24: Pole and zero locations for estimated plant after $10000 s$ and nonlinear plant.

As before,the error between the plant and the observer output does not converge to zero as shown in 3-25.

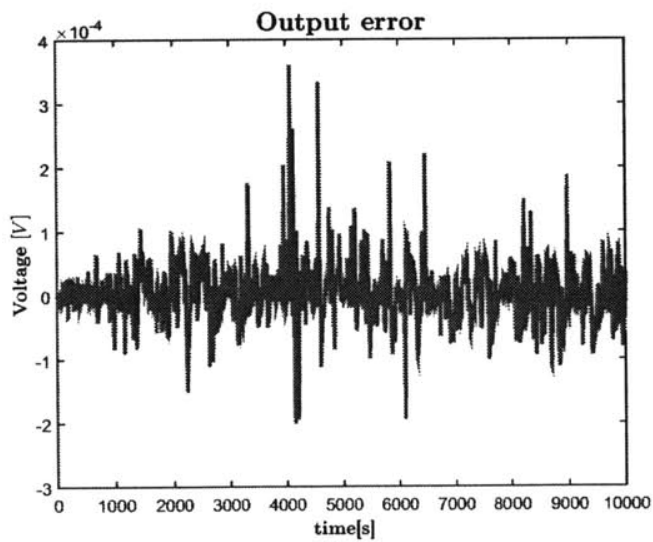

Figure 3-25: Error between plant output and estimated output.

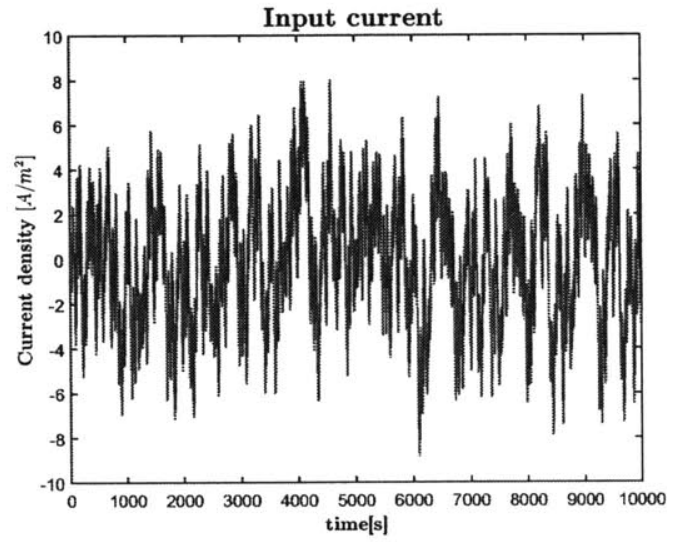

Figure 3-26: Current input to the linear plant.

We conclude that the linear adaptive observer with least square algorithm is only fitted for lower current, where nonlinearities marginally impact the input-output behavior of the cell. 


\subsection{Adaptive estimator for a two cathode material plant}

Li-ion intercalation cells used in EVs have usually a single insertion material on anode side, such as a carbon lattice $L i_{x} C_{6}$. In contrast to the anode, various cathode chemistries containing multiple insertion materials have been developed. Modeling such a cell with any of the above models, would be based on approximating the cathode as single material with lumped properties of the two materials. To provide a more accurate cell behavior, we suggest to keep the assumptions made for the single particle and extend the SPM to contain separate governing equations for each cathode material. The model description is derived in section 3.3.1. Like in the single material case, we further simplify the model to be linear in section 3.3.2. Based on the simplified model we deploy the extended adaptive observer to estimate the parameters of a multi-chemistry cell in the last section of this chapter.

\subsubsection{The Single Particle Model for a cathode with two inser- tion materials}

For the following derivation we use the subscript $l=1,2$ to denote the respective cathode material. First, the cell dynamics, i.e. the solid li-ion diffusion, have to be extended. We describe the lithium ion diffusion through the solid by Fick's law for each cathode material separately

$$
\frac{\partial c_{s, l}^{+}(r, t)}{\partial t}=\frac{1}{r^{2}} \frac{\partial}{\partial r}\left(D_{s, l}^{+} r^{2} \frac{\partial c_{s, l}^{+}(r, t)}{\partial r}\right) .
$$

We make a clear distinction between the surface fluxes of the two material and formulate boundary conditions as

$$
\begin{gathered}
\left.\frac{\partial c_{s, l}^{+}}{\partial r}\right|_{r=R_{p, l}^{+}}=-\frac{j_{n, l}^{+}}{D_{s, l}^{+}} \\
\left.\frac{\partial c_{s, l}^{+}}{\partial r}\right|_{r=0}=0
\end{gathered}
$$


and initial conditions as

$$
c_{s, l}^{+}(r, 0)=c_{s, l}^{+, 0}(r)
$$

Diffusion for the single material anode is described as shown in eq.(3.49) by

$$
\frac{\partial c_{s}^{-}(r, t)}{\partial t}=\frac{1}{r^{2}} \frac{\partial}{\partial r}\left(D_{s}^{-} r^{2} \frac{\partial c_{s}^{-}(r, t)}{\partial r}\right)
$$

with boundary conditions

$$
\begin{array}{r}
\left.\frac{\partial c_{s}^{-}}{\partial r}\right|_{r=R_{p}^{-}}=\frac{I(t)}{D_{s}^{-} F a^{-} L^{-}} \\
\left.\frac{\partial c_{s}^{-}}{\partial r}\right|_{r=0}=0
\end{array}
$$

and initial conditions

$$
c_{s}^{-}(r, 0)=c_{s}^{-, 0}(r)
$$

In order to satisfy Faraday's law, the current caused by the total surface flux of both materials in the cathode has to be equal to the applied cell current

$$
-I(t)=F a_{1}^{+} j_{n, 1}^{+} L^{+}+F a_{2}^{+} j_{n, 2}^{+} L^{+}
$$

This algebraic equation has to be fulfilled at any time and therefore, introduces a constraint on our system. A further constraint is introduces into the system by equating the two potentials of the materials on cathode side. By using the Butler volmer kinetics, we know that

$$
\begin{array}{r}
\phi_{s, 1}^{+}(t)=\frac{2 R T}{F} \sinh ^{-1}\left(\frac{j_{n, 1}^{+}}{2 r_{e f f, 1}^{+} \sqrt{c_{e}^{0} c_{s s, 1}^{+}(t)\left(c_{s, \text { max }}^{+}-c_{s s, 1}^{+}(t)\right)}}\right) \\
+U_{1}^{+}\left(c_{s s, 1}^{+}(t)\right)+R_{S E I, 1}^{+} F j_{n, 1}^{+}
\end{array}
$$


and

$$
\begin{array}{r}
\phi_{s, 2}^{+}(t)=\frac{2 R T}{F} \sinh ^{-1}\left(\frac{j_{n, 2}^{+}}{2 r_{e f f, 2}^{+} \sqrt{c_{e}^{0} c_{s s, 2}^{+}(t)\left(c_{s, \text { max }}^{+}-c_{s s, 2}^{+}(t)\right)}}\right) \\
+U_{2}^{+}\left(c_{s s, 2}^{+}(t)\right)+R_{S E I, 2}^{+} F j_{n, 2}^{+} .
\end{array}
$$

As both materials are not electrically insulated from each other, their potentials have to be on the same level

$$
g(t)=\phi_{s, 1}^{+}\left(j_{n, 1}^{+}(t), c_{s s, 1}^{+}(t)\right)-\phi_{s, 2}^{+}\left(j_{n, 2}^{+}(t), c_{s s, 2}^{+}(t)\right)=0
$$

We now substitute eq.(3.86) into the equation above and obtain

$$
g(t)=\phi_{s, 1}^{+}\left(j_{n, 1}^{+}(t), c_{s s, 1}^{+}(t)\right)-\phi_{s, 2}^{+}\left(-\frac{I}{F a_{2}^{+} L^{+}}-\frac{a_{1}^{+}}{a_{2}^{+}} j_{n, 1}^{+}, c_{s s, 2}^{+}(t)\right)=0
$$

This equation constrains the solution of the PDEs in Eqs.().

Finally, the cell voltage is defined by

$$
\begin{aligned}
V(t)=U_{1}^{+}\left(c_{s s, 1}^{+}(t)\right)-U^{-}\left(c_{s s}^{-}(t)\right) & \\
+ & \frac{2 R T}{F}\left[\sinh ^{-1}\left(\frac{j_{n, 1}^{+}}{2 r_{e f f, 1}^{+} \sqrt{c_{e}^{0} c_{s, 1}^{+}\left(R_{p}, t\right)\left(c_{s, \max }^{+}-c_{s, 1}^{+}\left(R_{p}, t\right)\right)}}\right)\right. \\
& \left.\quad-\sinh ^{-1}\left(\frac{I}{2 a^{-} L^{-} F r_{e f f}^{-} \sqrt{c_{e}^{0} c_{s s}^{-}(t)\left(c_{s, \max }^{-}-c_{s s}^{-}(t)\right)}}\right)\right] \\
& +R_{S E I, 1}^{+} F j_{n, 1}^{-}-R_{S E I}^{-} \frac{I}{a^{-} L^{-}}
\end{aligned}
$$

\subsubsection{Simplified model for two cathode materials}

As in the single material case, we can simplify the two cathode material cell model by applying volume-avering projections to the solid diffusion PDEs. We can use the volume averaging projections described in section 4.2 .4 to simplify the solid diffusion 
partial differential equations to

$$
\begin{aligned}
& \frac{\partial}{\partial t} \bar{c}_{s}(t)=-3 \frac{j^{-}(t)}{R_{p}^{-}} \\
& \frac{d}{d t} \bar{j}_{s}^{-}=-\frac{30}{\left(R_{p}^{-}\right)^{2}} D_{s}^{-} \bar{j}_{s}^{-}-\frac{45}{\left(2 R_{p}^{-}\right)^{2} F L^{-} a^{-}} I \\
& \frac{d}{d t} \bar{c}_{s, 1}^{+}=-\frac{3}{R_{p, 1}^{+}} j_{n, 1}^{+} \\
& \frac{d}{d t} \bar{j}_{s, 1}^{+}=-\frac{30}{\left(R_{p, 1}^{+}\right)^{2}} D_{s, 1}^{+} \bar{j}_{s, 1}^{+}-\frac{45}{\left(2 R_{p, 1}^{+}\right)^{2}} j_{n, 1}^{+} \\
& \frac{d}{d t} c_{s, 2}^{+}=\frac{3}{R_{p, 2}^{+}} \frac{a_{1}^{+}}{a_{2}^{+}} j_{n, 1}^{+}+\frac{3}{R_{p, 2}^{+} F L^{+} a_{2}^{+}} I \\
& \frac{d}{d t} \bar{j}_{s, 2}^{+}=-\frac{30}{\left(R_{p, 2}^{+}\right)^{2}} D_{s, 2}^{+} \bar{j}_{s, 2}^{+}+\frac{45}{\left(R_{p, 2}^{+}\right)^{2}} \frac{a_{1}^{+}}{a_{2}^{+}} j_{n, 1}^{+}+\frac{45}{\left(R_{p, 1}^{+}\right)^{2} F L^{+} a_{2}^{+}} I
\end{aligned}
$$

where $(\bar{\bullet})$ denotes the volume averaged values. If side reactions and loss of active material are neglected, then the conservation of recyclable lithium is defined by

$$
\frac{4}{3} \pi\left(R^{-}\right)^{3} \overline{c_{s}}+\frac{4}{3} \pi\left(R_{1}^{+}\right)^{3} \overline{c_{s, 1}^{+}}+\frac{4}{3} \pi\left(R_{2}^{+}\right)^{3} \overline{c_{s, 2}^{+}}=m_{L i}
$$

The parameter $m_{L i}$ denotes the total Lithium mass in the cell and is assumed to be unknown. The state $\bar{c}_{s}^{-}$can therefore always be expressed in terms of the cathode concentrations $\bar{c}_{s, 1}^{+}$and $\bar{c}_{s, 2}^{+}$and we can define the state vector as

$$
\boldsymbol{x}^{T}=\left[\begin{array}{llllll}
x_{1} & x_{2} & x_{3} & x_{4} & x_{5} & x_{6}
\end{array}\right]=\left[\begin{array}{llllll}
\bar{j}_{s}^{-} & \bar{c}_{s, 1}^{+} & \bar{j}_{s, 1}^{+} & \bar{c}_{s, 2}^{+} & \bar{j}_{s, 2}^{+} & j_{n, 1}^{+}
\end{array}\right]
$$

As shown in the previous section, the system (3.92) is constrained by the algebraic equation (3.90). For small deviations of $(\mathbf{x}, u)$ from a certain operating point $\left(\boldsymbol{x}_{0}, u_{0}\right)$, we can linearly approximate the equation (3.90) by

$$
F=\left.\frac{\partial g}{\partial \boldsymbol{x}}\right|_{\boldsymbol{x}=\boldsymbol{x}_{0}, u=u_{0}} \Delta \boldsymbol{x}+\left.\frac{\partial g}{\partial \boldsymbol{u}}\right|_{\boldsymbol{x}=\boldsymbol{x}_{0}, \boldsymbol{u}=u_{0}} \Delta \boldsymbol{u}+g\left(\boldsymbol{x}_{0}, u_{0}\right)=0
$$


We choose the operation point $\left(\boldsymbol{x}_{0}, u_{0}\right)$ to be

$$
\boldsymbol{x}_{\mathbf{0}}=\left[\begin{array}{lllllll}
\bar{c}_{s, 0}^{-} & \overline{j_{s, 0}^{-}} & \bar{c}_{s, 1,0}^{+} & \bar{j}_{s, 1,0}^{+} & \bar{c}_{s, 2,0}^{+} & \bar{j}_{s, 2,0}^{+} & j_{n, 1,0}^{+}
\end{array}\right]=\left[\begin{array}{lllllll}
\bar{c}_{s, 0}^{-} & 0 & \bar{c}_{s, 1,0}^{+} & 0 & \bar{c}_{s, 2,0}^{+} & 0 & 0
\end{array}\right]
$$

and

$$
u_{0}=0
$$

at which $\overrightarrow{c_{s, 1,0}^{+}}$and $\vec{c}_{s, 2,0}^{+}$are such that $\mathcal{U}_{1}^{+}\left(\bar{c}_{s, 1,0}^{+}\right)=\mathcal{U}_{2}^{+}\left(\vec{c}_{s, 2,0}^{+}\right)$. This operating point corresponds to the case of a cell in equilibrium, i.e. there are no fluxes in the cell. For this operating point $F$ becomes

$$
\begin{aligned}
F=\frac{\partial U_{1}^{+}}{\partial c_{s, 1}^{+}} \Delta x_{2}+\frac{8 R_{p, 1}^{+}}{35} \frac{\partial U_{1}^{+}}{\partial c_{s, 1}^{+}} \Delta x_{3}-\frac{\partial U_{2}^{+}}{\partial c_{s, 2}^{+}} \Delta x_{4}-\frac{8 R_{p, 2}^{+}}{35} \frac{\partial U_{2}^{+}}{\partial c_{s, 2}^{+}} \Delta x_{5} \\
+\left(\frac{R T}{F \sqrt{c_{e}^{0} c_{s, 1}^{+}\left(c_{s, \max , 1}-c_{s, 1}^{+}\right)} r_{e f f, 1}^{+}}+R_{S E I, 1} F-\frac{R_{p, 1}^{+}}{35 D_{s, 1}^{+}} \frac{\partial U_{1}^{+}}{\partial c_{s, 1}^{+}}\right. \\
+\frac{a_{1}^{+}}{a_{2}^{+}}\left(-\frac{R_{p, 2}^{+}}{35 D_{s, 2}^{+}} \frac{\partial U_{2}^{+}}{\partial c_{s, 2}^{+}}+\frac{R T}{r_{e f f, 2}^{+} F \sqrt{c_{e}^{0} c_{s, 2}^{+}\left(c_{s, \max , 2}^{+}-c_{s, 2}^{+}\right)}}+R_{S E I, 2}^{+} F\right) \Delta x_{6} \\
+\left(-\frac{R_{p, 2}^{+}}{35 F D_{s, 2}^{+}} \frac{\partial U_{2}^{+}}{\partial c_{s, 2}^{+}}+\frac{R T}{r_{e f f, 2}^{+} F^{2} \sqrt{c_{e}^{0} c_{s, 2}^{+}\left(c_{s, \max , 2}^{+}-c_{s, 2}^{+}\right)}}+R_{S E I, 2}^{+}\right) \frac{\Delta u}{a_{2}^{+}}
\end{aligned}
$$

This linearized algebraic equation can be solved for $\Delta x_{6}$, which can then be substituted into the dynamic equations for small deviations from $\left(\boldsymbol{x}_{0}, u_{0}\right)$. Hence, the minimal state vector fully describing the cell behavior is defined as

$$
\mathbf{x}_{m}^{T}=\left[\begin{array}{lllll}
\Delta \bar{j}_{s}^{-} & \Delta \bar{c}_{s, 1}^{+} & \Delta \bar{j}_{s, 1}^{+} & \Delta \bar{c}_{s, 2}^{+} & \Delta \bar{j}_{s, 2}^{+}
\end{array}\right]
$$

Small deviations of the cell voltage from equilibrium are defined by

$$
y=V(\mathbf{x}, t)-\left.V\left(\mathbf{x}_{0}, u_{0}\right) \approx \frac{\partial V}{\partial \mathbf{x}}\right|_{\mathbf{x}=\mathbf{x}_{0}, u=u_{0}} \Delta \mathbf{x}+\left.\frac{\partial V}{\partial u}\right|_{\mathbf{x}=\mathbf{x}_{0}, u=u_{0}} \Delta u,
$$


where

$$
V\left(\mathbf{x}_{0}, u_{0}\right)=\mathcal{U}_{1}^{+}\left(\bar{c}_{s, 1}^{+}\right)-\mathcal{U}^{-}\left(\bar{c}_{s, 1}^{+}, \bar{c}_{s, 2}^{+}, m_{L i}\right)
$$

is the open cell potential at equilibrium. If we substitute the equation for $\Delta x_{6}$ obtained from (3.95) into the equation (3.100), we obtain

$$
y=\mathbf{c}^{\mathbf{T}} \mathbf{x}_{\mathbf{m}}+d u
$$

where

$$
\mathbf{c}=\left[\begin{array}{c}
\frac{\partial \mathcal{U}^{-}}{\partial c_{s}^{-}} \frac{8 R_{p}^{-}}{35} \\
\left(\frac{\partial U_{1}^{+}}{\partial c_{s, 1}^{+}}\left(1-\frac{\delta}{\alpha}\right)+\frac{\partial \mathcal{U}^{-}}{\partial c_{s}^{-}} \frac{\left(R_{p, 1}^{+}\right)^{3}}{\left(R_{p}^{-}\right)^{3}}\right) \\
\left(1-\frac{\delta}{\alpha}\right) \frac{8 R_{p, 1}^{+}}{35} \frac{\partial U_{1}^{+}}{\partial c_{s, 1}^{+}} \\
\left(\frac{\partial \mathcal{U}^{-}}{\partial c_{s}^{-}} \frac{\left(R_{p, 2}^{+}\right)^{3}}{\left(R_{p}^{-}\right)^{3}}+\frac{\delta}{\alpha} \frac{\partial U_{2}^{+}}{\partial c_{s, 2}^{+}}\right) \\
\frac{\delta}{\alpha} \frac{8 R_{p, 2}^{+}}{35} \frac{\partial U_{2}^{+}}{\partial c_{s, 2}^{+}}
\end{array}\right]
$$

and

$$
d=\frac{1}{a^{-}}\left(-\frac{R_{f}^{-}}{L^{-}}+\frac{\partial \mathcal{U}^{-}}{\partial c_{s}^{-}} \frac{R_{p}^{-}}{35 F L^{-} D_{s}^{-}}-\frac{R T}{F^{2} L^{-} \sqrt{c_{e}^{0} c_{s}^{-}\left(c_{s, \text { max }}^{-}-c_{s}^{-}\right)}}\right)+\frac{\delta \beta}{\alpha}
$$

with

$$
\begin{gathered}
\alpha=\frac{R T}{F r_{e f f, 1}^{+} \sqrt{c_{e}^{0} c_{s, 1}^{+}\left(c_{s, \max , 1}-c_{s, 1}^{+}\right)}}+R_{S E I, 1} F-\frac{R_{p, 1}^{+}}{35 D_{s, 1}^{+}} \frac{\partial U_{1}^{+}}{\partial c_{s, 1}^{+}} \\
+\frac{a_{1}^{+}}{a_{2}^{+}}\left(-\frac{R_{p, 2}^{+}}{35 D_{s, 2}^{+}} \frac{\partial U_{2}^{+}}{\partial c_{s, 2}^{+}}+\frac{R T}{r_{e f f, 2}^{+} F \sqrt{c_{e}^{0} c_{s, 2}^{+}\left(c_{s, \max , 2}^{+}-c_{s, 2}^{+}\right)}}+R_{f, 2}^{+} F\right) \\
\beta=-\frac{R T}{35 F D_{s, 2}^{+}} \frac{\partial U_{2}^{+}}{\partial c_{s, 2}^{+}}+\frac{R}{r_{e f f, 2}^{+} F^{2} \sqrt{c_{e}^{0} c_{s, 2}^{+}\left(c_{s, \max , 2}^{+}-c_{s, 2}^{+}\right)}}+R_{f, 2}^{+}
\end{gathered}
$$

and

$$
\delta=\frac{1}{\frac{F}{R T} \sqrt{c_{e}^{0} c_{s, 1}^{+}\left(c_{s, \max , 1}-c_{s, 1}^{+}\right)}}+R_{f, 1} F-\frac{1}{D_{s, 1}^{+}} .
$$




\subsubsection{Simulation setup and results}

Our goal is to estimate parameters of a $\mathrm{LiCoO}_{2}, \mathrm{LiMnO}_{2}-\mathrm{LiC}_{6}$ cell with parameters defined in table A.2. We use the adaptive observer with a feed-through term as shown in section 3.1.2 combined with the recursive least square algorithm for parameter updates. As plant we choose the simplified two cathode material cell model. Parameters of interest are identified to be

$$
\theta=\left[\begin{array}{lllllll}
R_{p, 1}^{+} & R_{p, 2}^{+} & R_{p}^{-} & m_{L i} & D_{s, 1}^{+} & D_{s, 2}^{+} & D_{s}^{-}
\end{array}\right]
$$

The same plant properties shown for the single particle model for a single cathode material are valid for the two cathode material case. Observability of the plant is weak for flat regions of the OCV curve, where the feedthrough term dominates the plant input-output function. Moreover, the plant is marginally stable and exhibits dynamics on timescales of different order of magnitude. We will show that the adaptive observer is capable of dealing those complications.

Initial states of the plant are chosen to be $S O C_{a}=0.1, S O C_{c, 1}=0.8010$ and $S O C_{c, 2}=$ 0.69, where $c, 1$ denotes the $\mathrm{LiCoO}_{2}$ cathode material and $c, 2$ the $\mathrm{LiMnO}_{2}$ cathode material. The cell starts from equilibrium, at which both cathode materials have the same open circuit potential. It is then subjected to a superposition of harmonic inputs shown in 3-34 with frequencies defined in A.4, which creates a persistently exciting signal. Initial conditions of the estimated parameters are chosen to be off by $50 \%$. The initial estimate of the cell states have a $10 \%$ error. Filter values of the observer are chosen to be near the initial estimates for plant poles with

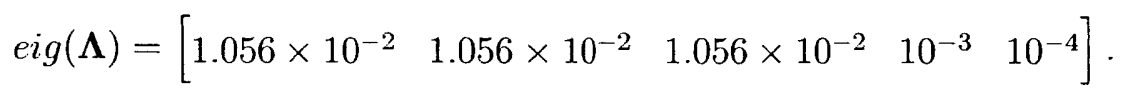

The initial gain for the recursive least square algorithm is $\Gamma_{0}=10^{10} \times \mathbf{I}$ and the forgetting factor is $\beta=10^{-10}$. We improve the numerical condition of the RLS by including an additional constant positive definite gain matrix $\boldsymbol{\Gamma}_{c}$. This matrix can be obtained by integrating $\dot{\Gamma}_{\mathbf{c}}=\sqrt{\hat{\omega} \hat{\omega}^{\mathbf{T}}}$ over a collection of prior generated data. 
The simulation results are validated by comparing input output behavior in Bode diagrams, pole and zero locations and analyzing parameter transients. Parameter estimates shown in 3-27 and 3-28 converge as expected after 5000s. Convergence takes longer for the two cathode material cell compared to the single material cell due to the augmented order of the plant.

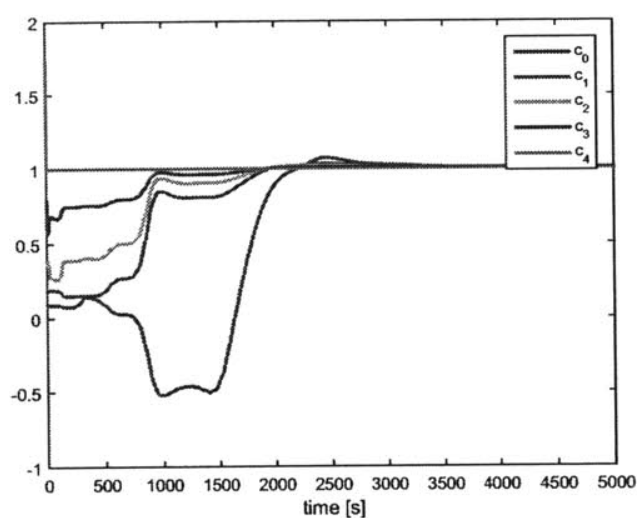

Figure 3-27: Parameter estimates for $t=$ $0-5000 s$. All parameters estimates have been normalized by their actual parameter value.

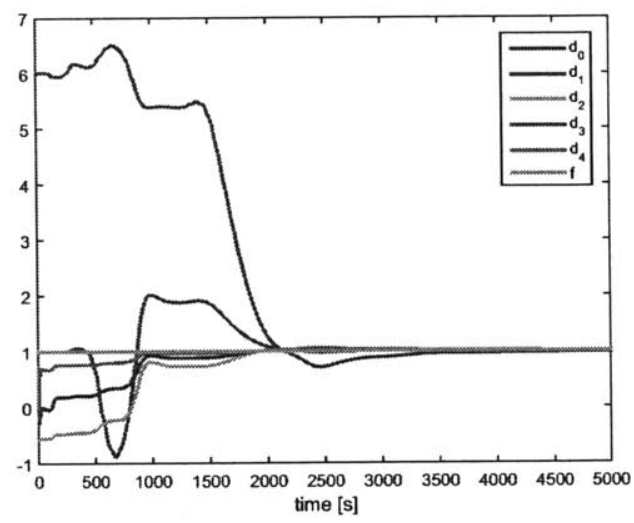

Figure 3-28: Parameter estimates for $t=$ $0-5000 s$. All parameters estimates have been normalized by their actual parameter value.

Comparing the Bode diagrams in figures 3-29-3-30 and pole and zero locations in figures 3-31-3-28 after 5000s shows perfect matching of the estimated plant with the actual plant. 


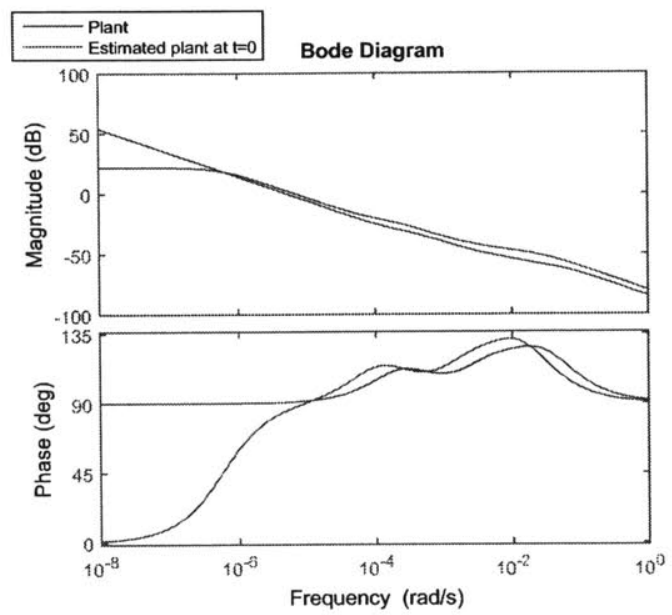

Figure 3-29: Bode diagram for initial plant estimation and linear plant.

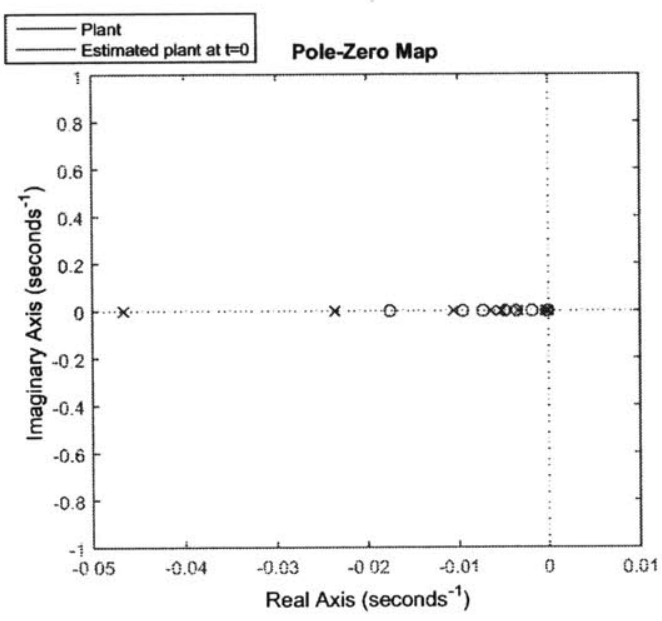

Figure 3-31: Pole and zero locations for initial plant estimate and linear plant.

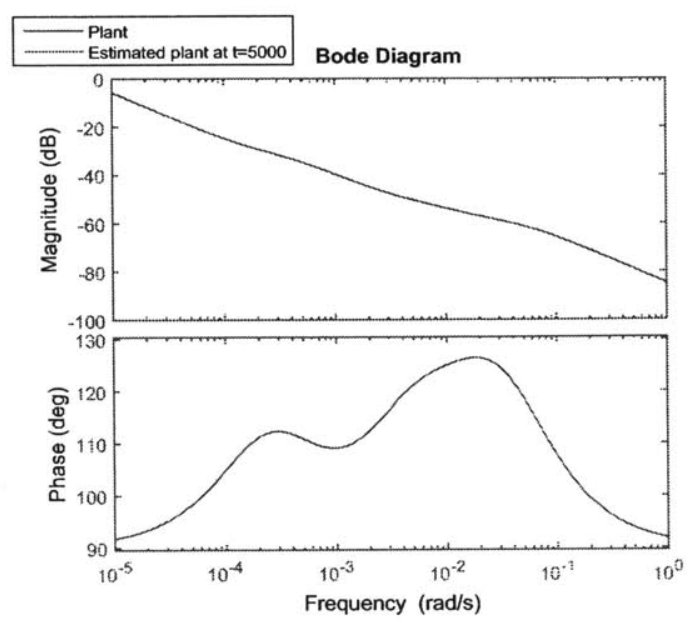

Figure 3-30: Bode diagram for estimated plant after $5000 \mathrm{~s}$ and linear plant.

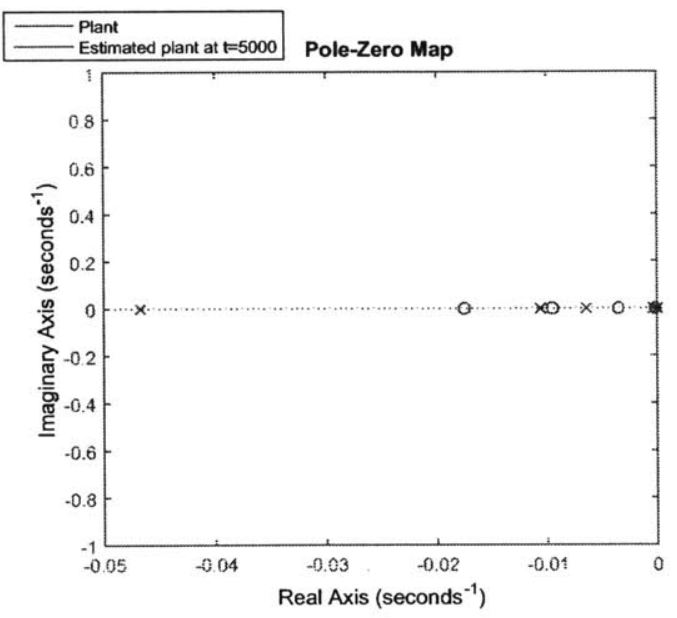

Figure 3-32: Pole and zero locations for estimated plant after $5000 \mathrm{~s}$ and linear plant.

As predicted by the stability analysis, the observer is globally stable if applied to the linear plant. The output error as defined in (3.34) converges to zero as shown in $3-33$. 


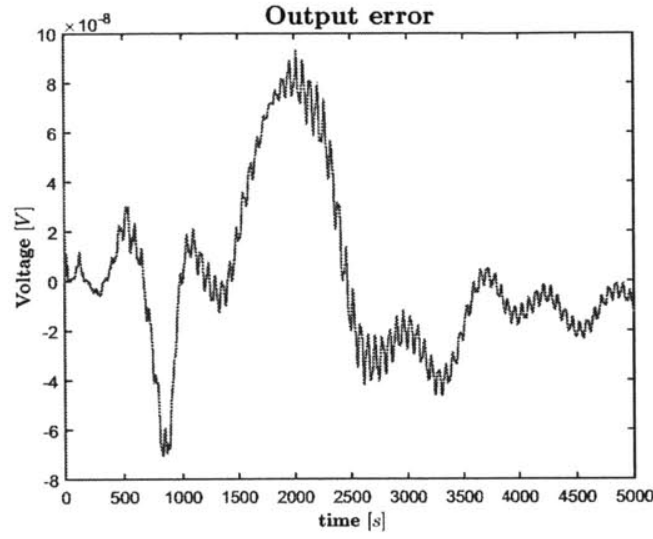

Figure 3-33: Error between plant output and estimated output.

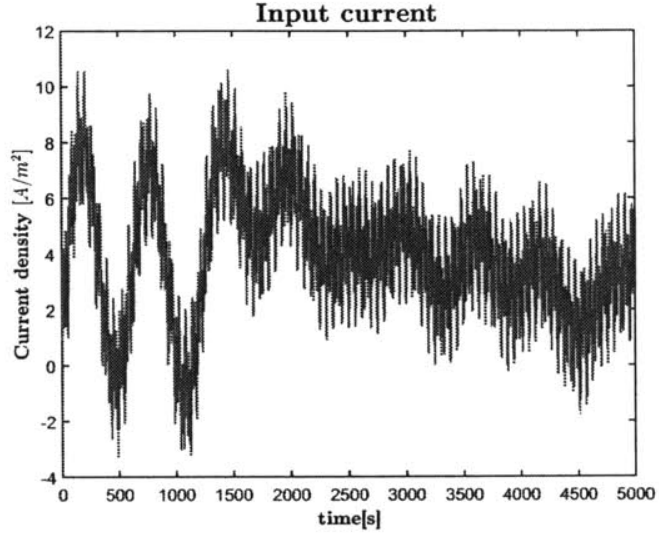

Figure 3-34: Current input to the linear plant. 


\section{Chapter 4}

\section{Control-oriented electrochemical lithium ion cell models}

According to [17] there exist four main categories of lithium ion intercalation cell models - Empirical models, electrochemical models, multi-physics models and molecular/atomistic models. While the majority of these models are primarily used for systems engineering, i.e. optimizing cell properties with help of simulations, an appropriate model has to be found for battery management systems (BMS). It has to capture the principal dynamics of the system, while being simple enough to be applicable to real time usage. Furthermore, it is preferable to avoid any loss of physical insight, as is the case with equivalent circuit models. Therefore, our goal is to derive a control-oriented reduced order model that succinctly captures the principal dynamics, and retains all of the physical insight.

Our starting point is a core electrochemical model presented in [5], denoted as Pseudo 2-Dimensional model (P2D). This is the most common physics-based model in the literature thus far [17]. We will introduce simplifications into the P2D model using concepts from the Absolute Nodal Coordinate Formulation (ANCF) proposed by Shabana et al. (see [28] for example). As we will show in this report, our simplified model (denoted as the ANCF model) is low order yet sufficiently accurate for capturing the main dynamics of a Lithium-ion battery while preserving insight into the dominant 
physical phenomena.

In Section 2.1, we first describe the P2D model. In Section 4.2, we present the ANCF model, which is a simplification of the P2D model using Galerkin projections. Validation of the ANCF model and comparison to the SPM model are shown in Section 4.4.

\subsection{Pseudo two dimensional model}

We consider a typical intercalation battery with a schematic as shown in Fig.(4-1). The electrode compartments are assumed to have two phases - the electrode material which is assumed to be a porous solid and the electrolyte, a solution containing the Lithium ions [2]. The separator in between the two electrode consists of electrically insulating material and the electrolyte, which only allows Lithium ions to flow through it. The dynamics of the system is assumed to vary only in one direction $x$, whose axis is perpendicular to the collector surfaces. This assumption is justified for the case where the z-y cross-section area is much bigger than the length of the cell in $\mathrm{x}$-direction, which is the case for typical cells with length ratios around $1: 10^{3}$ [2]. The distribution and form of porous electrode material is approximated by assuming pseudo spheres along the x-direction, in which the lithium ions intercalate. The transport dynamics in those spheres, which contain the lattice sites for the intercalation of $\mathrm{Li}$ ions, are formulated for a pseudo radial direction $\mathrm{r}$, as shown in Fig.(4-1). The P2D model incorporates the electrochemical kinetics at the electrodes, solid- and electrolyte potentials, transport phenomena and currents, which will be individually discussed in the following subsections. 


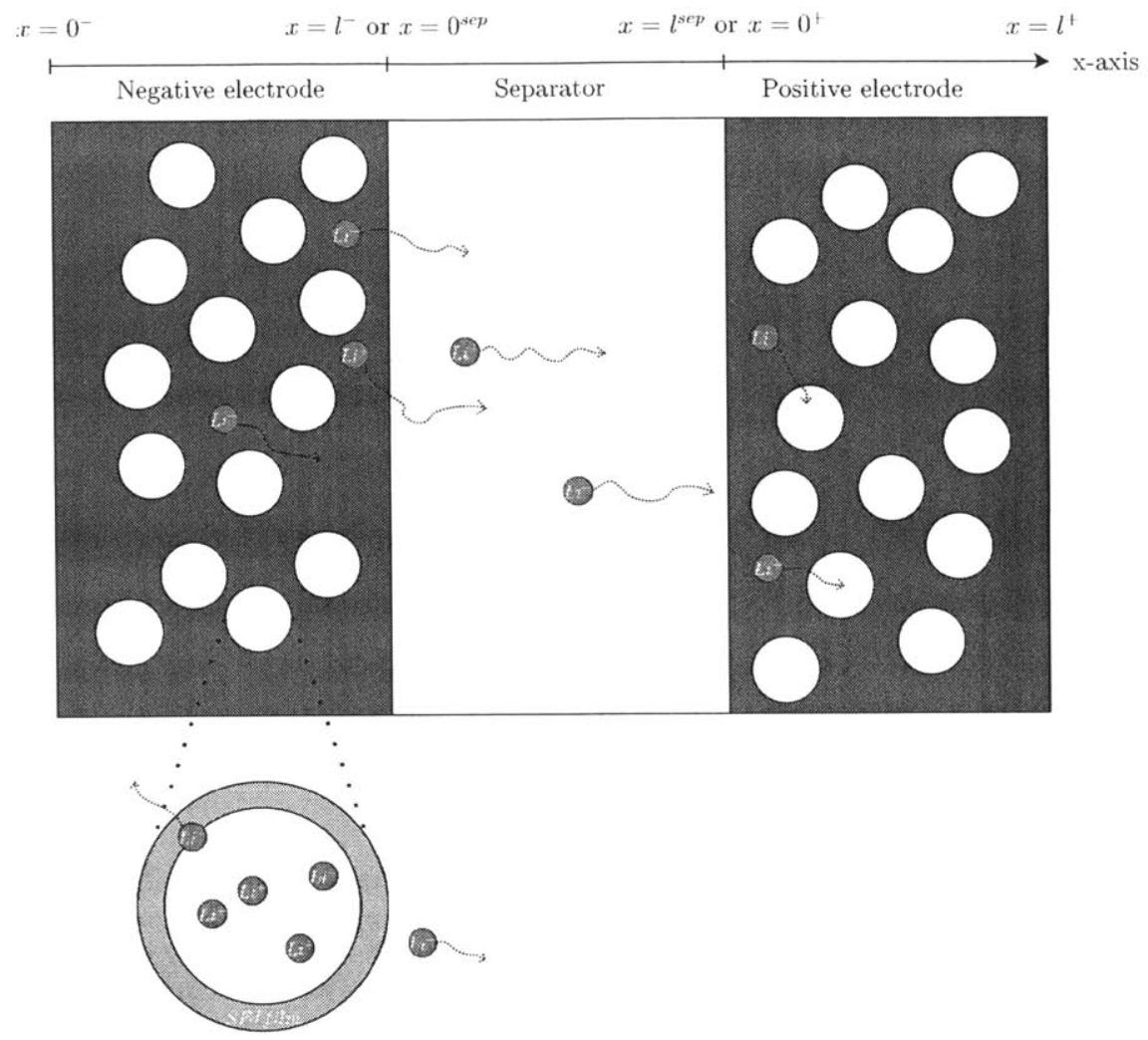

Figure 4-1: Schematic illustration of a Lithium ion intercalation cell during discharge with a magnifying view on a solid electrode particle.

Note that we use the superscript $i=+,-$, sep to denote the compartment. + will be used for the positive electrode, sep for the separator and - for the negative electrode. For reasons of simplicity we introduce special notations for the $x$ location of the boundaries of the electrodes and separator. The negative electrode has a collectorelectrode interface at $x=0$, which we will address as $x=0^{-}$. The separator-electrode interface at $x=l_{n}$, will be addressed by $x=l^{-}$or $x=0^{s e p}$. Further, the separatorpositive electrode boundary at $x=l^{-}+l^{\text {sep }}$, is addressed as $x=l^{\text {sep }}$ or $x=0^{+}$. Finally, the collector surface location of the positive electrode at $x=l^{-}+l^{+}+l^{\text {sep }}$ is denoted by $x=l^{+}$. First, we formulate the governing equations for the electrode compartments and then for the separator. 


\subsubsection{Current in the electrode}

The total current flowing trough the electrode compartment is the electrolyte current $i_{e}$ caused by the Li-ion flux in the electrolyte and the solid current $i_{s}$ originating from the electron flow in the solid material. This total has to equal the current applied to the cell $I(t)=i_{e}+i_{s}$. Notice, that for the configuration chosen in Fig.(4-1), $I(t)$ is positive while discharging and negative during charging processes. The total flux $J(x, t)$ of Li-ions in the electrolyte is related to $i_{e}$ by Faraday's law

$$
i_{e}=J(x, t) F
$$

where F is called the Faraday's constant. This total flux increases/decreases at every position $\mathrm{x}$ by the flux $j(x, t)$ exiting/entering the fictive sphere at $\mathrm{x}$. Thus the change of $i_{e}$ in x-direction can be expressed by

$$
\frac{\partial i_{e}(x, t)}{\partial x}=F \frac{\partial J(x, t)}{\partial x}=a^{i} F j(x, t)
$$

which is also referred to as law of conservation of charge. The parameter $a^{i}=(1-$

$\left.\epsilon_{e}^{i}-\epsilon_{f}^{i}\right) \frac{4 \pi\left(R_{p}^{i}\right)^{2}}{\frac{1}{3} \pi\left(R_{p}^{i}\right)^{3}}=\left(1-\epsilon_{e}^{i}-\epsilon_{f}^{i}\right) \frac{3}{R_{p}^{i}}$, where $R_{p}^{i}$ is the particle radius, is called the specific interfacial area. We further define $\epsilon_{e}^{i}$ as the volume fraction of the electrolyte in compartment $\mathrm{i}$ and $\epsilon_{f}^{i}$ as the volume fraction of the binding or filling material. It represents the total electrode material surface at $\mathrm{x}$. At the collector surface the electrolyte current $i_{e}$ is zero, because of the impermeability of the collectors to Liions. Analog, the solid current $i_{s}$ at the boundary between electrode and separator equals zero, because of the impermeability of the separator to electrons. Hence, the electrolyte current in the separator is equal to the applied current to the cell. The boundary conditions for $i_{e}$ are

$$
\begin{gathered}
i_{e}\left(0^{-}, t\right)=0, \quad i_{e}\left(l^{-}, t\right)=I(t) \\
i_{e}\left(0^{+}, t\right)=I(t), \quad i_{e}\left(l^{+}, t\right)=0 .
\end{gathered}
$$




\subsubsection{Potential in the solid electrode}

The potential in the solid material originating from the current in the solid material is described by Ohm's law

$$
\frac{\partial \phi_{s}(x, t)}{\partial x}=\frac{i_{s}(x, t)}{\sigma_{e f f}^{i}}=\frac{i_{e}(x, t)-I(t)}{\sigma_{e f f}^{i}}
$$

where $\sigma_{\text {eff }}^{i}$ is the effective electronic conductivity defined by

$$
\sigma_{e f f}^{i}=\sigma^{i}\left(1-\epsilon_{e}^{i}-\epsilon_{f}^{i}\right)
$$

In the equation above $\sigma^{i}$ is defined as the pure material electronic conductivity. There are no explicit boundary conditions posed to the potential in the solid electrode. However, the missing boundary conditions can be replaced by using the conditions for the current at boundary surfaces as derived above, as will be shown later on.

\subsubsection{Potential in the electrolyte of the electrode}

The potential in the electrolyte can be derived by using the condition of phase equilibrium for the charged species $\mathrm{Li}$ in changing phases, combined with the definition of potential difference between phases of identical composition. For the full derivation the reader is referred to [13] p.49-50. The electrolyte potential can be expressed as

$$
\frac{\partial \phi_{e}(x, t)}{\partial x}=-\frac{i_{e}(x, t)}{\kappa^{i}(x, t)}+\frac{2 R T}{F}\left(1-t_{c}^{0}\right)\left(1+\frac{\operatorname{dln} f_{c / a}(x, t)}{\operatorname{dln} c_{e}}\right) \frac{\partial \ln c_{e}(x, t)}{\partial x},
$$

where $f_{c / a}(x, t)=f_{c / a}\left(c_{e}(x, t)\right)$ is the mean molar activity coefficient and depends on the electrolyte concentration. The parameter $\kappa^{i}(x, t)=\kappa^{i}\left(c_{e}(x, t)\right)$ is the ionic conductivity of the electrolyte, which also depends on the electrolyte concentration. For our analysis we will assume $f_{c / a}$ and $\kappa^{i}$ to be constant over space. $t_{c}^{0}$ is the transference number of the cations. The temperature $T$ is modeled as a constant for the P2D model. However, modeling of $T$ has been already investigated by different works [19] [14]. $R$ is the universal gas constant. The boundary conditions are 
continuity conditions at the boundaries between separator and electrode.

$$
\begin{gathered}
\phi_{e}\left(l^{-}, t\right)=\phi_{e}\left(0^{s e p}, t\right) \\
\phi_{e}\left(l^{s e p}, t\right)=\phi_{e, p}\left(0^{+}, t\right)
\end{gathered}
$$

and a arbitrary potential reference set point

$$
\phi_{e}\left(0^{-}, t\right)=0
$$

\subsubsection{Transport in the electrolyte of the electrode}

The concentration of Li-ions in the electrolyte at point $\mathrm{x}$ changes because of diffusion,

which is caused by a concentration gradient in $\mathrm{x}$-direction, and the exiting/entering flux of Li-ions from the solid phase. Therefore, the governing equations are

$$
\begin{aligned}
\epsilon_{e}^{i} \frac{\partial c_{e}(x, t)}{\partial t} & =\frac{\partial}{\partial x}\left(D_{\text {eff }}^{i} \frac{\partial c_{e}(x, t)}{\partial x}\right)+\frac{1}{F} \frac{\partial\left(t_{a}^{0} i_{e}(x, t)\right)}{\partial x} \\
& =\frac{\partial}{\partial x}\left(D_{e f f}^{i} \frac{\partial c_{e}(x, t)}{\partial x}\right)+t_{a}^{0} a^{i} j(x, t)
\end{aligned}
$$

where $t_{a}^{0}$ is the transference number for the anions and

$$
D_{\text {eff }}^{i}=D\left(\epsilon_{e}^{i}\right)^{b r u g g}
$$

is the effective diffusion coefficient of the electrolyte [20] defined by Brugg's law. The Brugg factor is usually assumed to be brugg $=1.5$.

Due to the impermeability of the collectors to lithium ions, the flux is zero at the collector surface and therefore

$$
\left.\frac{\partial c_{e}}{\partial x}\right|_{x=0}=0,\left.\quad \frac{\partial c_{e}}{\partial x}\right|_{x=l^{+}}=0 .
$$


Further boundary conditions result from flux continuity at the electrode-separator interfaces

$$
\begin{aligned}
& \left.\epsilon_{e}^{-}\left(D_{e}^{-} \frac{\partial c_{e}}{\partial x}\right)\right|_{x=l^{-}}=\left.\epsilon_{e}^{s e p}\left(D_{e}^{s e p} \frac{\partial c_{e}}{\partial x}\right)\right|_{x=0^{s e p}} \\
& \left.\epsilon_{e}^{s e p}\left(D_{e}^{s e p} \frac{\partial c_{e}}{\partial x}\right)\right|_{x=l^{\text {sep }}}=\left.\epsilon_{e}^{-}\left(D_{e}^{-} \frac{\partial c_{e}}{\partial x}\right)\right|_{x=0^{+}}
\end{aligned}
$$

and concentration continuity

$$
c_{e}\left(l^{-}, t\right)=c_{e}\left(0^{s e p}, t\right), \quad c_{e}\left(l^{s e p}, t\right)=c_{e}\left(0^{+}, t\right) .
$$

\subsubsection{Transport in the solid electrode}

The transport of Li-ions within the solid electrode can be mainly attributed to diffusion. Therefore, the change of solid concentration can be described by Fick's law for spherical coordinates

$$
\frac{\partial c_{s}(x, r, t)}{\partial t}=\frac{1}{r^{2}} \frac{\partial}{\partial r}\left(D_{s}^{i} r^{2} \frac{\partial c_{s}(x, r, t)}{\partial r}\right)
$$

with $D_{s}^{i}$ being the solid diffusion coefficient. Notice that $D_{s}^{i}$ can be dependent on the concentration of the solid. Schmidt et al. [19] have shown for a cell containing two different insertion materials that the solid diffusion coefficient is dependent on the state of charge averaged over the particle volume.

The boundary conditions result from the definition of flux at the surface of the pseudo sphere

$$
\left.\frac{\partial c_{s}}{\partial r}\right|_{r=R_{p}}=-\frac{1}{D_{s}^{i}} j(x, t),
$$

and symmetry at the center

$$
\left.\frac{\partial c_{s}}{\partial r}\right|_{r=0}=0
$$

As initial condition we set the concentration throughout the whole sphere to a constant initial value

$$
c_{s}(r, x, 0)=c_{s}^{0, i}
$$


Diffusion between adjacent particles is neglected, because of a high solid phase diffusive impedance between them [2].

\subsubsection{Reaction kinetics of the electrode}

The reaction rate at the surface of the spheres induces a loss, the so called kinetic overpotential $\eta_{s}$, which can be expressed as the potential difference between electrode solid and electrolyte versus the open circuit potential of the electrode.

$$
\eta_{s}(x)=\phi_{s}(x, t)-\phi_{e}(x, t)-\mathcal{U}\left(c_{s s}(x, t)\right)-F R_{S E I} j(x, t) .
$$

An additional voltage/power loss is caused by the solid-electrolyte interphase shown in Fig.(4.40), which acts as a an additional impedance. This interphase is formed on anodes, made e.g. by lithium or carbon, by the reduction of the electrolyte and is generally unstable [6]. The lattice expands and contracts during charging and discharging cycles causing the layer to break and reform. Measurements of the healing rate are very difficult to obtain [6]. For reasons of simplicity, the impedance is modeled as a constant $R_{S E I}$.

The flux exiting at the surface of the sphere, which equals the reaction rate, is related to the above mentioned overpotential by the Butler-Volmer equations [13]

$$
j(x, t)=i_{0}(x, t)\left[\exp \left(\frac{\alpha_{a} F}{R T} \eta(x, t)\right)-\exp \left(\frac{-\alpha_{a} F}{R T} \eta(x, t)\right)\right]
$$

with $\alpha_{a}$ and $\alpha_{c}$ denoting transport coefficients. $i_{0}$ represents the exchange current density

$$
i_{0}(x, t)=k^{i} c_{e}(x, t)^{\alpha_{a}}\left(c_{s, \max }-c_{s s}(x, t)\right)^{\alpha_{a}} c_{s s}(x, t)^{\alpha_{c}},
$$

where $k^{i}$ is the reaction rate constant for electrode $i$ and $c_{s s}(x, t)=c_{s}\left(x, R_{p}, t\right)$ is the surface concentration of the particle. 


\subsubsection{Governing equation in the separator}

For the separator, we have to formulate the governing equations in the electrolyte, which are the transport and the electrolyte potential equations.

\section{Electrolyte potential}

The electrolyte current $i_{e}$ in the separator is equal to the applied current to the cell, because its insulating property to electrons. The governing equation simplifies therefore to

$$
\frac{\partial \phi_{e}(x, t)}{\partial x}=-\frac{I(t)}{\kappa^{i}(x, t)}+\frac{2 R T}{F}\left(1-t_{c}^{0}\right)\left(1+\frac{\operatorname{dln} f_{c / a}(x, t)}{\operatorname{dln} c_{e}}\right) \frac{\partial \ln c_{e}(x, t)}{\partial x} .
$$

\section{Transport in the electrolyte}

In the separator compartment the Ion flux stays constant and hence the concentration only changes due to diffusion

$$
\frac{\partial c_{e}(x, t)}{\partial t}=\frac{\partial}{\partial x}\left(D_{e f f}^{i} \frac{\partial c_{e}(x, t)}{\partial x}\right)
$$

\subsection{The ANCF Li-ion cell model}

The basic idea of the ANCF model is to divide the $\mathrm{x}$-space equally into elements and approximate the solutions for these elements with a function. This approximating function has to be in accordance with the physical phenomena of the cell. These phenomena are the continuity of fluxes and concentrations throughout the cell. Therefore, we rely on the absolute nodal coordinate formulation first proposed by [28], who applied it to the analysis of highly flexible beams. While this formulation preserves continuity of stress and deformation in the case of beams, it conserves the flux and concentration continuity for our electrochemical model and hence, fulfills the conservation of mass. Furthermore, the discretization allows us to account for the local nature of the electrolyte transport phenomena, which is caused by the out-/influx of lithium ions from the solid particles. This advantage becomes extremely important 
e.g. at high currents and sharp current changes, where strong fluxes/concentration gradients locally occur near boundaries. Different authors [20] [19] proposed approximating functions for a whole compartment, but did not include a spacial discretization. Subramanian et al. [20] used fourth order polynomials for each electrode and the separator. While low currents and thus weak changes in $\mathrm{x}$ direction of the variables could be captured by this approach, higher currents could not be approximated. Further increasing the order of the polynomial can not be numerically executed, because of the preceding analytical calculations linked to it. We therefore suggest to use the so called absolute nodal coordinate polynomial which closely resembles a finite element approach. In addition, to its physical consistency, the ANCF model is applicable for real time applications due to its high numerical performance. As will be shown in the last section, it relies on a low number of independent variables to converge to a highly accurate solution. In the following sections, we first introduce the absolute nodal coordinate formulation polynomial and then reformulate the dynamic equations of the cell by applying the method of weighted residuals.

\subsubsection{The absolute nodal coordinate formulation}

We present the absolute nodal coordinate fomulation for the concentration of the electrolyte and note that the other variables are approximated in the same manner. In addition, we will be deriving the function only for one spatial compartment.

We first introduce $N$ nodes to equally divide the $\mathrm{x}$-space of the electrode or separator in $N-1$ elements with length $l^{\prime}=\frac{l}{N-1}$. For every element $i$ we use a local coordinate defined by

$$
s_{i}=\frac{x-(i-1) l^{\prime}}{l^{\prime}}
$$

and define an element addressing function

$$
\chi_{i}(x)= \begin{cases}1 & \text { if } x \in\left[l^{\prime} i, l^{\prime}(i+1)\right] \\ 0 & \text { else }\end{cases}
$$


The solution of the concentration profile for element $i$ is approximated by a cubic polynomial

$$
c_{e, i}\left(s_{i}, t\right)=\alpha_{i}(t)+\beta_{i}(t) s_{i}+\gamma_{i}(t) s_{i}^{2}+\delta_{i}(t) s_{i}^{3}
$$

and therefore, the concentration profile in one compartment is defined as

$$
c_{e}(x, t)=\sum_{i=1}^{N-1} \chi_{i} c_{e, i}\left(s_{i}, t\right)
$$

By using a third order polynomial the concentration gradient and value are guaranteed to be continuous. In fact, the node values and derivatives of the element can be used to obtain a physical representative version of the cubic polynomial. By using

$$
\begin{gathered}
c_{e, i}(0, t)=c_{e}^{i}, \quad c_{e, i}(1, t)=c_{e}^{i+1} \\
\left.\frac{\partial c_{e, i}\left(s_{i}, t\right)}{\partial s_{i}}\right|_{s_{i}=0}=l^{\prime} \frac{\partial c_{e}^{i}}{\partial x},\left.\quad \frac{\partial c_{e, i}\left(s_{i}, t\right)}{\partial s_{i}}\right|_{s_{i}=1}=l^{\prime} \frac{\partial c_{e}^{i+1}}{\partial x}
\end{gathered}
$$

we can manipulate Eq.(4.29) to

$$
\begin{aligned}
c_{e, i}\left(s_{i}, t\right)=c_{e}^{i}(t)\left(1-3 s_{i}^{2}+2 s_{i}^{3}\right)+ & c_{e}^{i+1}(t)\left(3 s_{i}^{2}-2 s_{i}^{3}\right) \\
& +\frac{\partial c_{e}^{i}}{\partial x} l^{\prime}\left(s_{i}-2 s_{i}^{2}+s_{i}^{3}\right)+\frac{\partial c_{e}^{i+1}}{\partial x} l^{\prime}\left(-s_{i}^{2}+s_{i}^{3}\right)
\end{aligned}
$$

Notice, that the time coefficients are equal to the degrees of freedom of our approach. Furthermore, we define a vector notation of the polynomial as

$$
c_{e, i}\left(s_{i}, t\right)=\mathbf{c}_{i}(t) \mathbf{s}\left(s_{i}\right),
$$

where

$$
\mathbf{c}_{i}(t)=\left[\begin{array}{c}
c_{e}^{i}(t) \\
\frac{\partial c_{e}^{i}}{\partial x} l^{\prime} \\
c_{e}^{i+1} \\
\frac{\partial c_{e}^{i+1}}{\partial x} l^{\prime}
\end{array}\right]
$$


denotes the time coefficient vector and

$$
\mathbf{s}\left(s_{i}\right)=\left[\begin{array}{c}
1-3 s_{i}^{2}+2 s_{i}^{3} \\
s_{i}-2 s_{i}^{2}+s_{i}^{3} \\
3 s_{i}^{2}-2 s_{i}^{3} \\
-s_{i}^{2}+s_{i}^{3}
\end{array}\right]
$$

is called the profile or shape vector. The polynomial function introduced for the electrolyte concentration will analogously be used for the solid concentration and the reaction rates of the particles. We will now fit this approximation to the real solutions of the partial differential algebraic equations for the battery cell.

\subsubsection{The Method of Weighted Residual}

In this chapter, we provide a short introduction to the method of weighted residuals (MWR), which will subsequently be used to fit the ANC function to the real solutions of the partial differential algebraic equations. If we use the partial space differential operator $\mathcal{D}(\bullet)$, then we can express a partial differential equation for a variable $u(x, t)$ as

$$
\mathcal{D}(u(x, t))=p(u(x, t), x, t)
$$

or

$$
\mathcal{D}(u(x, t))-p(u(x, t), x, t)=0
$$

where $p(u(x, t), x, t)$ can be a nonlinear function of partial time derivatives, $x$ and/or $t$. The equation above is only valid for the exact solution $u(x, t)$. However, if we use an approximation $u(x, t) \cong \tilde{u}(x, t)$, then Eq.(4.38) is not equal to zero any more but results in a so called residual

$$
\mathcal{D}(\tilde{u}(x, t))-p(\tilde{u}(x, t), x, t)=R(x, t) .
$$

In our case the approximation $\tilde{u}(x, t)$ is the absolute nodal coordinate formulation presented above. The aim of the weighted residual method is to determine values 
of the coefficients/degrees of freedom of the approximating function, which minimize the residual over a certain weighting integral. The weighting integral is defined as

$$
\int w^{i}(x) R(x, t) \mathrm{d} x=0 \quad \text { for } i=1, \ldots, N_{c o e f f}
$$

where $w^{i}(x)$ are the weighting functions and $N_{\text {coeff }}$ is the maximum degree of freedoms equivalent to the number of coefficients of our absolute nodal coordinate formulation. There exist different options for the choice of $w^{i}(x)$ to which the residual is made orthogonal. For our derivation we will use the Galerkin collocation and the Orthogonal collocation. The first method is defined by

$$
w^{i}(x)=\frac{\partial \tilde{u}(x, t)}{\partial a^{i}}
$$

with $a^{i}$ being the time coefficients. For the ANCF we obtain $w^{i}=(\mathbf{s})_{i}$ or $\mathbf{w}=\mathbf{s}$ if expressed as vectors. The orthogonal collocation on the other hand uses dirac functions as weighting function

$$
w^{i}(x)=\delta\left(x-d^{i}\right)
$$

where $d^{i}$ can be chosen to be the solutions of the Gauss-Legendre polynom. The Galerkin collocation method is the preferred method, if there exists an analytical solutions to Eq.(4.40). Notice, that because of the discretization we applied for the $\mathrm{ANCF}$, the integral has to be evaluated for every element.

\subsubsection{Transport in the electrolyte}

In order to obtain the differential algebraic equations for the time coefficients of the absolute nodal coordinate formulation for one element, we apply the method of weighted residuals to the governing equations of the electrolyte concentration. First, 
we define the governing equation using Eq.(4.11) as

$$
\epsilon_{e} \frac{\partial c_{e}(x, t)}{\partial t}-\frac{\partial}{\partial x}\left(D_{e f f}^{i} \frac{\partial c_{e}(x, t)}{\partial x}\right)-t_{a}^{0} a j(x, t)=0
$$

Substituting the absolute nodal coordinate polynomial defined in Eq.(4.34) for the electrolyte concentration $c_{e}^{i}\left(s_{i}, t\right)=\mathbf{s}^{\mathrm{T}} \mathbf{c}_{e}^{i}$ and the surface flux $j^{i}\left(s_{i}, t\right)=\mathbf{s}^{\mathrm{T}} \mathbf{j}^{i}$ into the equation above results in the residual

$$
R\left(s_{i}, t\right)=\mathbf{s}^{\mathrm{T}} \frac{\mathrm{d} \mathbf{c}_{e}^{i}}{\mathrm{dt}}-\frac{D_{e f f}}{\epsilon_{e} l^{\prime}} \frac{\mathrm{d}^{2} \mathbf{s}^{\mathrm{T}}}{\mathrm{ds}^{2}} \mathbf{c}_{e}^{i}-\frac{t_{a}^{0}}{\epsilon_{e}} a \mathbf{s}^{\mathrm{T}} \mathbf{j}^{i}
$$

Further, we apply the Galerkin collocation with $\mathbf{w}=\mathbf{s}$ to obtain

$$
\begin{aligned}
\int_{0}^{1} \mathbf{w} R\left(s_{i}, t\right) \mathrm{d} s_{i} & =\int_{0}^{1} \mathbf{s} R\left(s_{i}, t\right) \mathrm{d} s_{i} \\
& =\int_{0}^{1} \mathbf{s s}^{\mathrm{T}} \mathrm{d} s_{i} \frac{\mathrm{dc}_{e}^{i}}{\mathrm{dt}}-\frac{D_{e f f}}{\epsilon_{e} l^{\prime}} \int_{0}^{1} \mathbf{s}^{\mathrm{d}^{2} \mathbf{s}^{\mathrm{T}}} \frac{\mathrm{ds} \mathrm{s}_{\mathrm{i}}{ }^{2}}{\mathrm{~d}} s_{i} \mathbf{c}_{e}^{i}-\frac{t_{a}^{0}}{\epsilon_{e}} a \int_{0}^{1} \mathbf{s s}^{\mathrm{T}} \mathrm{d} s_{i} \mathbf{j}^{i} \\
& =\mathbf{M} \frac{\mathrm{dc}_{e}^{i}}{\mathrm{dt}}-\frac{D_{e f f}}{\epsilon_{e} l^{\prime}} \mathbf{K c}_{e}^{i}-\frac{t_{a}^{0}}{\epsilon_{e}} a \mathbf{M} \mathbf{j}^{i} \\
& =0
\end{aligned}
$$

where

$$
\mathbf{M}=\int_{0}^{1} \mathbf{S s}^{\mathrm{T}} \mathrm{d} s_{i}=\left[\begin{array}{cccc}
\frac{13}{35} & \frac{11}{210} & \frac{9}{70} & -\frac{13}{420} \\
\frac{11}{210} & \frac{1}{105} & \frac{13}{420} & -\frac{1}{140} \\
\frac{9}{70} & \frac{13}{420} & \frac{13}{35} & -\frac{11}{210} \\
-\frac{13}{420} & -\frac{1}{140} & -\frac{11}{210} & \frac{1}{105}
\end{array}\right]
$$

and

$$
\mathbf{K}=\int_{0}^{1} \mathrm{~s} \frac{\mathrm{d}^{2} \mathbf{s}^{\mathrm{T}}}{\mathrm{ds}^{2}} \mathrm{~d} s_{i}=\left[\begin{array}{cccc}
-\frac{6}{5} & -\frac{11}{10} & \frac{6}{5} & -\frac{1}{10} \\
-\frac{1}{10} & -\frac{2}{15} & \frac{1}{10} & \frac{1}{30} \\
\frac{6}{5} & \frac{1}{10} & -\frac{6}{5} & \frac{11}{10} \\
-\frac{1}{10} & \frac{1}{30} & \frac{1}{10} & -\frac{2}{15}
\end{array}\right]
$$

The no flux condition at each collector predefines two coefficients, i.e. the derivatives at the boundaries are set to zero. 


\subsubsection{Transport the solid electrode}

It has been shown [21] that approximating the solid concentration in $\mathbf{r}$-direction by a three parameter polynomial is sufficient for low and high currents. We therefore apply the concepts used to simplify the partial differential equation for the solid concentration presented by Subramanian V.R. et al. [21]. A polynomial profile with three-parameters

$$
c_{s}(x, r, t)=a(x, t)+b(x, t)\left(\frac{r^{2}}{\left(R_{p}^{i}\right)^{2}}\right)+d(t)\left(\frac{r^{4}}{\left(R_{p}^{i}\right)^{4}}\right)
$$

is used to approximate the concentration of the pseudo sphere. In order to transform the coefficient into a physical representation we introduce the volume-averaged flux by

$$
\bar{q}(x, t)=\int_{r=0}^{R_{p}^{i}} 3 \frac{r^{2}}{\left(R_{p}^{i}\right)^{2}}\left(\frac{\mathrm{d}}{\mathrm{dr}} c_{s}(x, r, t)\right) \mathrm{d}\left(\frac{r}{R_{p}^{i}}\right),
$$

the volume averaged concentration by

$$
\bar{c}_{s}(x, t)=\int_{r=0}^{R_{p}^{i}} 3 \frac{r^{2}}{\left(R_{p}^{i}\right)^{2}} c(r, t) \mathrm{d}\left(\frac{r}{R_{p}^{i}}\right)
$$

and the surface concentration by $c_{s s}(x, t)=c_{s}\left(x, R_{p}^{i}, t\right)$.

Substituting Eq.(4.51) into Eqs(4.52-4.53) results in

$$
\begin{gathered}
a(t)=\frac{39}{4} c_{s s}(x, t)-3 \bar{q}(x, t) R_{p}^{i}-\frac{35}{4} \bar{c}_{s}(x, t) \\
b(t)=-35 c_{s s}(x, t)+10 \bar{q}(x, t) R_{p}^{i}+35 \bar{c}_{s}(x, t) \\
d(t)=\frac{105}{4} c_{s s}(x, t)-7 \bar{q}(x, t) R_{p}^{i}-\frac{105}{4} \bar{c}_{s}(x, t) .
\end{gathered}
$$

If the concentration profile is now substituted into Eq.(4.18) we receive the two linear algebraic differential equations

$$
\frac{\partial}{\partial t} \bar{c}_{s}(x, t)=-3 \frac{j(x, t)}{R_{p}^{i}}
$$




$$
\frac{\partial}{\partial t} \bar{q}(x, t)=-30 \frac{D_{s}^{i}}{\left(R_{p}^{i}\right)^{2}} \bar{q}(x, t)-\frac{45}{\left(R_{p}^{i}\right)^{2}} j(x, t)
$$

and the nonlinear equation

$$
35 \frac{D_{s}^{i}}{R_{p}^{i}}\left[c_{s s}(x, t)-\bar{c}(x, t)\right]-8 D_{s}^{i} \bar{q}(x, t)=-j(x, t) .
$$

The average concentration and flux are further approximated by the absolute nodal coordinate formulation in x-direction. In this case, the MWR is not needed because of the linear and first order of the partial differential equations. In fact, we can use the principle of separation of variables, i.e. coefficients which are multiplied by the same shape function are equated. The resulting equations are

$$
\frac{\mathrm{dc}_{s}^{i}(t)}{\mathrm{dt}}=-3 \frac{\mathbf{j}^{i}(t)}{R_{p}^{i}}
$$

and

$$
\frac{\mathrm{d} \overline{\mathbf{q}}^{i}(t)}{\mathrm{dt}}=-30 \frac{D_{s}^{i}}{\left(R_{p}^{i}\right)^{2}} \overline{\mathbf{q}}^{i}(t)-\frac{45}{\left(R_{p}^{i}\right)^{2}} \mathrm{j}^{i}(t) .
$$

\subsubsection{Reaction kinetics at the electrode}

Before applying the method of weighted residuals for the surface flux/reaction kinetics, we will use the mathematical reformulation presented in [2]. They showed that the spatial partial differential equations $(4.23),(4.5),(4.7)$ can be expressed as one integral function depending on the solid surface concentration, electrolyte concentration and the reaction kinetics at the surface of the particles. We start by differentiating Eq.(4.5) and then substitute Eq.(4.2) which results in

$$
\frac{\partial^{2} \phi_{s}}{\partial x^{2}}=\frac{1}{\sigma_{e f f}} \frac{\partial i_{e}(x, t)}{\partial x}=\frac{a^{i} F j(x, t)}{\sigma_{e f f}^{i}}
$$

The solid potential is therefore a function of the double integral of the surface flux

$$
\phi_{s}(x, t)=\phi_{s}\left(\iint j(x, t) d x d x\right)
$$


Using the absolute nodal coordinate formulation for the surface flux $j_{i}\left(s_{i}, t\right)=\mathbf{s}^{\mathrm{T}} \mathbf{j}^{i}$ gives us the solid potential for element $i$

$$
\phi_{s, i}\left(s_{i}, t\right)=\frac{a F l^{\prime 2}}{\sigma_{e f f}} \int_{0}^{s_{i}} \mathrm{~s}^{\mathrm{T}}(\tilde{s}) \mathrm{d} \tilde{s} \mathrm{j}^{i}+\phi_{s}^{i}+\frac{\partial \phi_{s}^{i}}{\partial s} s_{i} .
$$

For the electrolyte potential we substitute $i_{e}=I(t)-i_{s}=I(t)-\frac{\partial \phi_{s}}{\partial x} \sigma_{e f f}^{i}$ into Eq.(4.7) and assume that mean molar activity is constant. It results

$$
\begin{aligned}
\frac{\partial \phi_{e}(x, t)}{\partial x} & =-\frac{i_{e}(x, t)}{\kappa_{e f f}^{i}(x, t)}+\frac{2 R T}{F}\left(1-t_{c}^{0}\right) \frac{\partial \ln c_{e}(x, t)}{\partial x} \\
& =-\frac{I(t)}{\kappa_{e f f}^{i}(x, t)}-\frac{\partial \phi_{s}(x, t)}{\partial x} \frac{\sigma_{e f f}}{\kappa_{e f f}^{i}(x, t)}+\frac{2 R T}{F}\left(1-\iota_{c}^{0}\right) \frac{1}{c_{e}(x, t)} \frac{\partial c_{e}(x, t)}{\partial x}
\end{aligned}
$$

Hence, the electrolyte potential is dependent on the electrolyte concentration, the cell current and the solid potential derivative, which is a function of the double integral of the surface flux

$$
\phi_{e}(x, t)=\phi_{e}\left(\iint j(x, t) d x d x, c_{e}(x, t), t\right)
$$

For our further analysis we assume that the ionic conductivity is constant. Inserting the absolute nodal coordinate formulation for the flux $j_{i}\left(s_{i}, t\right)=\mathbf{s}^{\mathrm{T}} \mathbf{j}^{i}$ and evaluating for element $i$, gives us

$\phi_{e, i}\left(s_{i}, t\right)=-\frac{I(t)}{\kappa_{e f f}} s_{i}-\left(\phi_{s, i}\left(s_{i}, t\right)-\phi_{s}^{i}\right) \frac{\sigma_{e f f}}{\kappa_{e f f}}+\frac{2 R T}{F}\left(1-t_{c}^{0}\right)\left(\ln \left(c_{e, i}\left(s_{i}, t\right)\right)-\ln \left(c_{e}^{i}\right)\right)+\phi_{e}^{i}$,

which, if we substitute Eq.(4.64), results in

$$
\begin{aligned}
\phi_{e, i}\left(s_{i}, t\right)=-\frac{I(t)}{\kappa_{e f f}} s_{i}-\left(\frac{a F l^{\prime 2}}{\sigma_{e f f}} \int_{0}^{s_{i}}\right. & \left.\mathbf{s}^{\mathrm{T}}(\tilde{s}, t) \mathrm{d} \tilde{s} \mathrm{j}^{i}+\frac{\partial \phi_{s}^{i}}{\partial s} s_{i}\right) \frac{\sigma_{e f f}}{\kappa_{e f f}} \\
& +\frac{2 R T}{F}\left(1-t_{c}^{0}\right)\left(\ln \left(c_{e, i}\left(s_{i}, t\right)\right)-\ln \left(c_{e}^{i}\right)\right)+\phi_{e}^{i}
\end{aligned}
$$


Using the relations from above, the overpotential for the electrode kinetics

$$
\eta_{s}(x)=\phi_{s}(x, t)-\phi_{e}(x, t)-\mathcal{U}\left(c_{s s}(x, t)\right)-F R_{S E I} j(x, t)
$$

is a function of

$$
\eta(x, t)=\eta\left(\iint j(x, t) d x d x, j(x, t), c_{s s}(x, t), c_{e}(x, t)\right)
$$

Finally, the electrode kinetics which are described by the Butler-Volmer kinetics in Eq.(4.23) are a nonlinear integral equation of $j(x, t)$

$$
\begin{aligned}
j(x, t)=i_{0}\left(c_{e}(x, t), c_{s s}(x, t)\right) & \\
& {\left[\exp \left(\frac{\alpha_{c} F}{R T} \eta\left(\iint j(x, t) d x d x, j(x, t), c_{s s}(x, t), c_{e}(x, t)\right)\right)\right.} \\
- & \left.\exp \left(\frac{-\alpha_{a} F}{R T} \eta\left(\iint j(x, t) d x d x, j(x, t), c_{s s}(x, t), c_{e}(x, t)\right)\right)\right] .
\end{aligned}
$$

We apply the method of weighted residuals to this integral equation to the integral equation. Unfortunately, no analytical solution can be found if the Galerkin colloction method is applied. In this case, we have to use the orthogonal collocation method. We force the residual of governing equation

$$
\begin{aligned}
& R\left(s_{i}, t\right)= i_{0}\left(c_{e, i}\left(s_{i}, t\right), c_{s s, i}\left(s_{i}, t\right)\right) \\
& {\left[\exp \left(\frac{\alpha_{c} F}{R T} \eta\left(\iint j_{i}\left(s_{i}, t\right) \mathrm{d} s_{i} \mathrm{~d} s_{i}, j_{i}\left(s_{i}, t\right), c_{s s, i}\left(s_{i}, t\right), c_{e, i}\left(s_{i}, t\right)\right)\right)\right.} \\
&-\exp \left(\frac{-\alpha_{a} F}{R T} \eta\left(\iint j_{i}\left(s_{i}, t\right) \mathrm{d} s_{i} \mathrm{~d} s_{i}, j_{i}\left(s_{i}, t\right), c_{s s, i}\left(s_{i}, t\right), c_{e, i}\left(s_{i}, t\right)\right)\right]-j_{i}\left(s_{i}, t\right) .
\end{aligned}
$$

to be equal to zero at four defined points of every element

$$
\int_{0}^{1} \delta\left(s_{i}-d_{j}\right) R\left(s_{i}, t\right) \mathrm{d} s_{i} \quad \text { for } j=1,2,3,4
$$


For our application we choose $d_{j}$ to be the integration points from the Gauss Legendre algorithm for a four point integration on the interval of $[0,1]$.

\subsection{Summary of the ANCF model}

The dynamics for one element $i$ is described by a system of differential algebraic equations given in Eq.(4.75)-Eq.(4.78) below.

$$
\begin{gathered}
\frac{\mathrm{d} \mathbf{c}_{e}^{i}}{\mathrm{dt}}=\frac{D_{e f f}^{j}}{\epsilon_{e}^{j} l^{\prime}} \mathbf{M}^{-1} \mathbf{K} \mathbf{c}_{e}^{i}+\frac{t_{a}^{0}}{\epsilon_{e}^{j}} a^{j} \mathbf{j}^{i} \\
\frac{\mathrm{d} \overline{\mathbf{c}}_{s}^{i}(t)}{\mathrm{dt}}=-3 \frac{\mathbf{j}^{i}(t)}{R_{p}^{j}} \\
\frac{\mathrm{d} \overline{\mathbf{q}}^{i}(t)}{\mathrm{dt}}=-30 \frac{D_{s}^{j}}{\left(R_{p}^{j}\right)^{2}} \overline{\mathbf{q}}^{i}(t)-\frac{45}{\left(R_{p}^{j}\right)^{2}} \mathbf{j}^{i}(t) \\
\mathbf{f}\left(\mathbf{c}_{e}^{i}(t), \overline{\mathbf{c}}_{s}^{i}(t), \overline{\mathbf{q}}^{i}(t), \mathbf{j}^{i}(t), t\right)=0
\end{gathered}
$$




\subsection{Validation of the ANCF model and comparison to SPM}

We first validate the ANCF model by subjecting it to two charging and discharging scenarios.The first test consists of continuous discharging with different C-rates between upper voltage cut-off limit and lower voltage limit, followed by relaxation for 30 minutes. The second test tests the model for repeated cycles of high current charging followed by high current discharging and relaxation. The ANCF model is validated against the DUALFOIL model, a high fidelity model based on the P2D formulation. The ANCF simulation is executed with two elements in each electrode and one element in the separator. Simulations are conducted on a $\mathrm{LiCoO}_{2} / \mathrm{LiC}_{6}$ cell with properties defined in table A.4. Three simplifications are made for the given parameters, in order to simulate the cell with the ANCF model. The ANCF model is derived from the governing equations, assuming that the electrolyte diffusion coefficient $D_{e}$ and the electrolyte ionic conductivity $\kappa$ are constant. Therefore, both are approximated by constant values for the simulation. The ionic conductivity coefficient is chosen to be $\kappa=0.74$ for all simulations, which is the value for a salt concentration

of $c_{r e f}=1000\left[\mathrm{~mol} / \mathrm{m}^{3}\right]$. The diffusion coefficient is chosen to be as small as possible, such that the sluggish behavior of the electrolyte can be observed. However, the coefficient had to be increased for higher discharge rates, due to premature cell deaths through fast depletion in the electrolyte. The simplified parameter values are listed in table 4.1. A better approximation could be potentially achieved if the diffusion coefficient is varied per element depending on the average salt concentration in the element. Finally, the thermal behavior of the cell is not included for the simulations and simulations are executed for isothermal conditions at $23 C$. Voltage limits are chosen to be $4.2 \mathrm{~V}$ and $2.5 \mathrm{~V}$ for this cell chemistry.

For the first case we denote the time instant, at which the cell voltage attains the lower cutoff voltage $t=t_{X}$. We compare electrolyte concentration distribution, electrolyte potential profile and the surface flux distribution for the time instants 


\begin{tabular}{|l|l|l|}
\hline & $1 C$ & $2 C$ \\
\hline$D_{e}$ & $1.5394 e-09$ & $1.4 e-10$ \\
\hline
\end{tabular}

Table 4.1: Diffusion coefficient values

$t=0.3 t_{X}, 0.5 t_{X}, 0.8 t_{X}, t_{X}, t_{X}+1$ min $, t_{X}+10 \mathrm{~min}, t_{X}+30 \mathrm{~min}$. The voltage profile for a $1 C$ discharge is shown in figure 4-2.

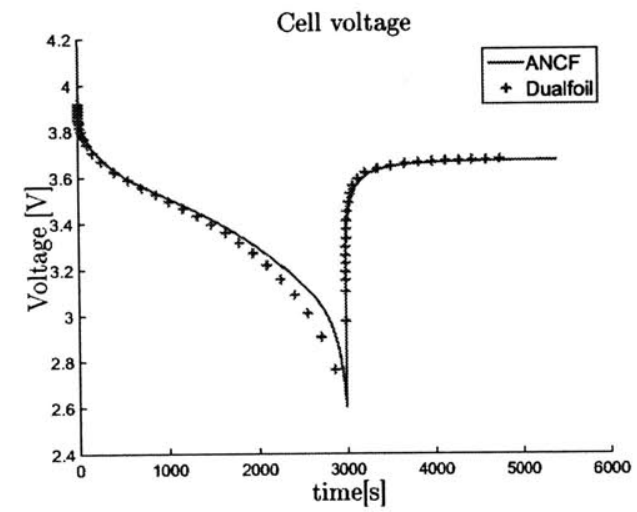

Figure 4-2: Cell voltage for a constant discharge of $1 C$ with $30 \mathrm{~min}$ relaxation after full discharge: ANCF model with one element per electrode compared to Dualfoil

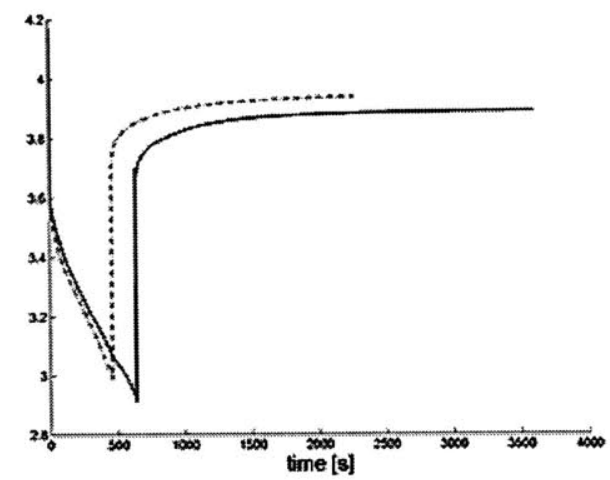

Figure 4-3: Cell voltage for a constant discharge of $2 C$ with $30 \mathrm{~min}$ relaxation after full discharge: ANCF model with one element per electrode compared to Dualfoil

Voltage profiles for the $1 C$ discharge match well, i.e. the ANCF voltage profile deviates less than $1 \%$ from the Dualfoil model. Internal state profiles of the cell for a constant discharge current of $1 C$ are shown in figure $4-4$ to $4-9$.

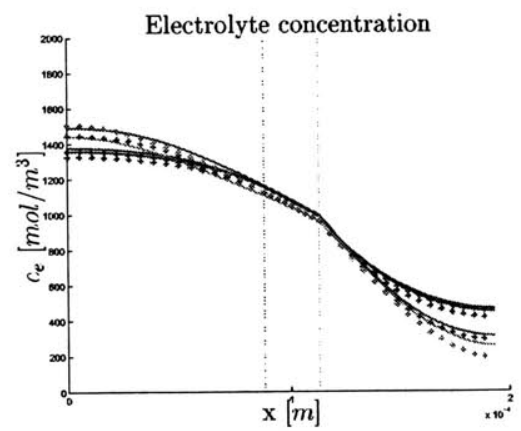

Figure 4-4: Electrolyte profiles before full discharge: ANCF model with one element per electrode compared to Dualfoil

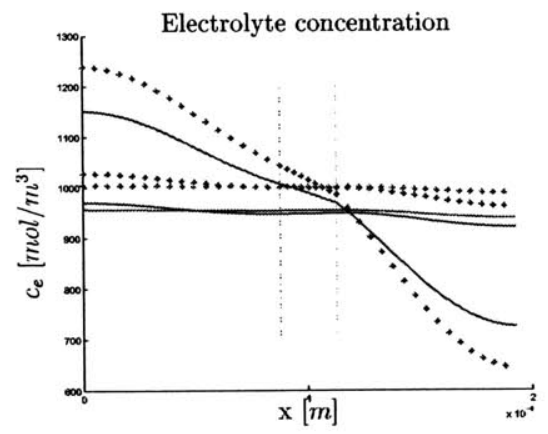

Figure 4-5: Electrolyte profiles during relaxation: ANCF model with one element per electrode compared to Dualfoil 


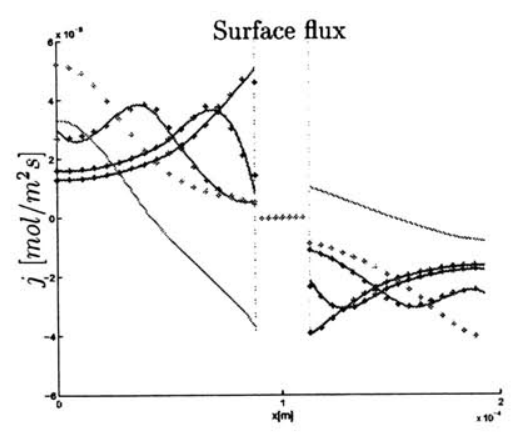

Figure 4-6: Surface fluxes before full discharge: ANCF model with one element per electrode compared to Dualfoil

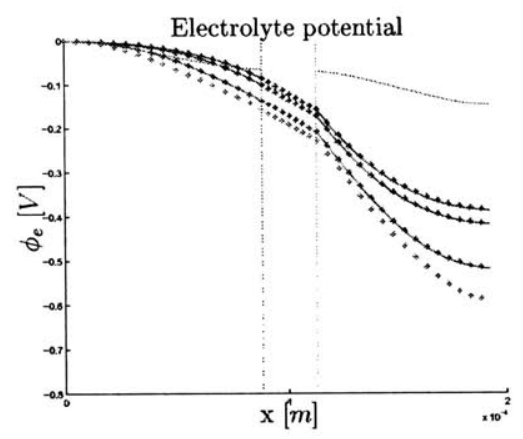

Figure 4-8: Electrolyte potential before full discharge: ANCF model with one element per electrode compared to Dualfoil

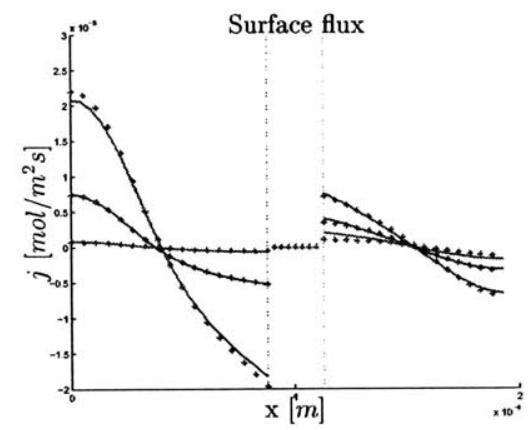

Figure 4-7: Surface fluxes after full discharge: ANCF model with one element per electrode compared to Dualfoil

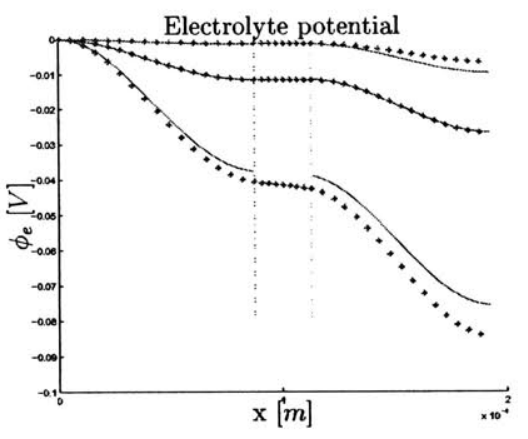

Figure 4-9: Electrolyte potential during relaxation: ANCF model with one element per electrode compared to Dualfoil

For the chosen cell, the limiting factor is transport in the electrolyte. Most of the Lithium ion intercalation into or out of the solid material occurs near to the separator, because of the fast reaction kinetics compared to the sluggish electrolyte transport. Therefore, the material at the separator interfaces gets emptied much faster, which causes early cell deaths/discharges. For these types of cells, where electrolyte transport dominates the cell behavior, we can see clear advantages in using the ANCF model. It simulates variations of solid and electrolyte concentration levels in $\mathrm{x}$-direction and therefore captures the excessive strains on the material near to the separator. Monitoring the local phenomena is useful, in order to prevent e.g. capacity fade by cracking of solid material. 
The voltage profile for the $2 C$ discharge is presented in figure 4-3. In the second simulation, where $D_{e}=1.4 e-10\left[\mathrm{~m}^{2} / \mathrm{s}\right]$ was used for a $2 C$ discharge, we can observe a premature cell death due to electrolyte depletion. The ANCF model predicts a different voltage profile in the latter case. This deviation is can be attributed to unprecise approximations of the electrolyte profile in the ANCF model. The electrolyte depletes first at the electrode boundary and locally creates a very sharp profile, which is not precisely captured by the ANCF as shown in figure 4-10.

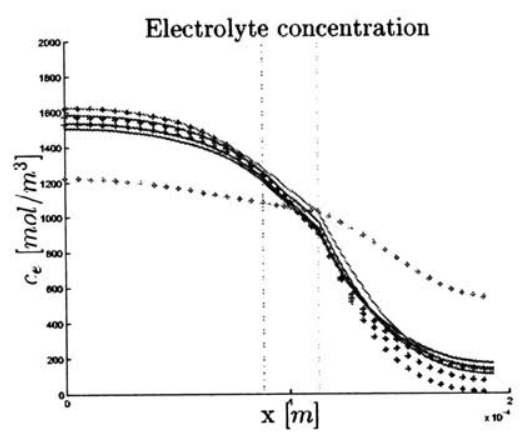

Figure 4-10: Electrolyte profiles before full discharge: ANCF model with one element per electrode compared to Dualfoil

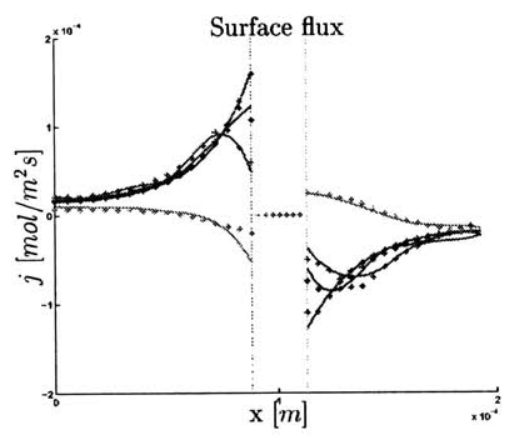

Figure 4-12: Surface fluxes before full discharge: ANCF model with one element per electrode compared to Dualfoil

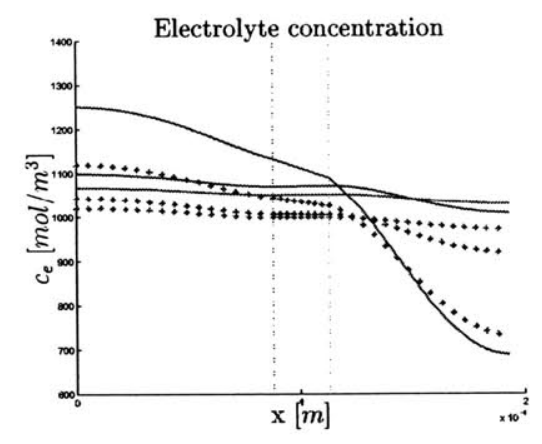

Figure 4-11: Electrolyte profiles during relaxation: ANCF model with one element per electrode compared to Dualfoil

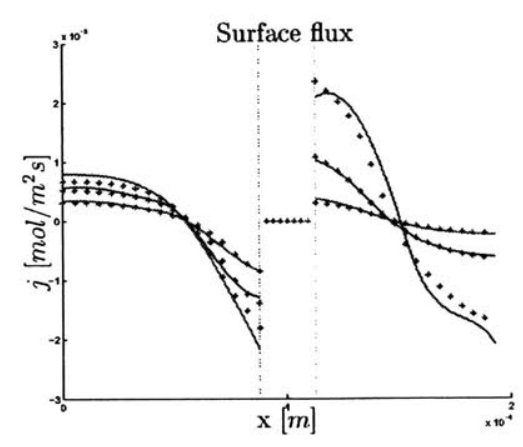

Figure 4-13: Surface fluxes after full discharge: ANCF model with one element per electrode compared to Dualfoil 


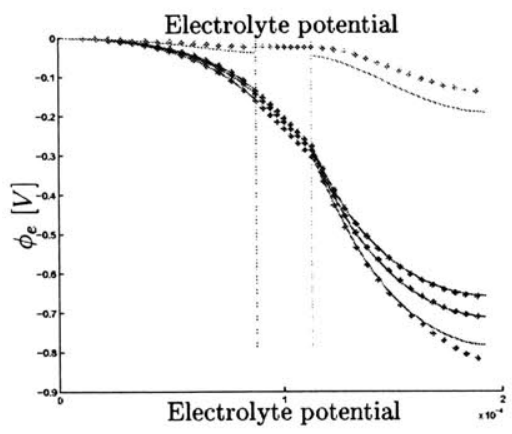

Figure 4-14: Electrolyte profiles before full discharge: ANCF model with one element per electrode compared to Dualfoil

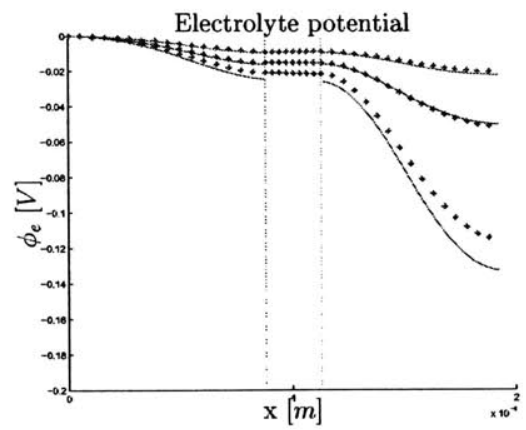

Figure 4-15: Electrolyte profiles during relaxation: ANCF model with one element per electrode compared to Dualfoil

For the second scenario we subject the cell to two different cycles of charge, discharge and rest periods as shown in fig.4-16 for $2 \mathrm{C}$ peak currents and in fig.4-18 for $4 \mathrm{C}$ peak currents. The cell voltage starts to deviate from the Dualfoil model at high currents after sudden changes in current, due to the simplifications made to the solid concentration equations. The simplified system obtained by volume-averaging projections in (4.60) and (4.61) can not precisely replicate solid diffusion at high currents.

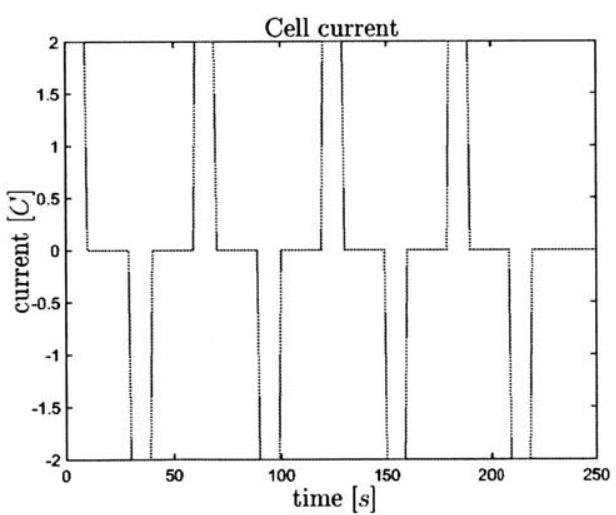

Figure 4-16: A series of charges and discharges at $2 \mathrm{C}$

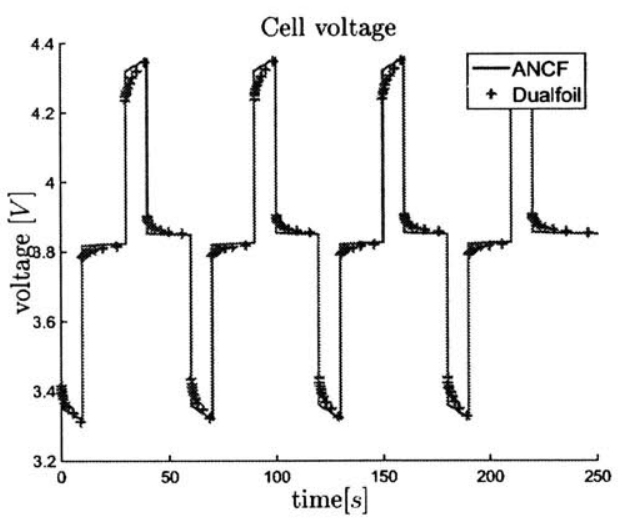

Figure 4-17: Cell voltage for the $2 \mathrm{C}$ discharge and charge cycle 


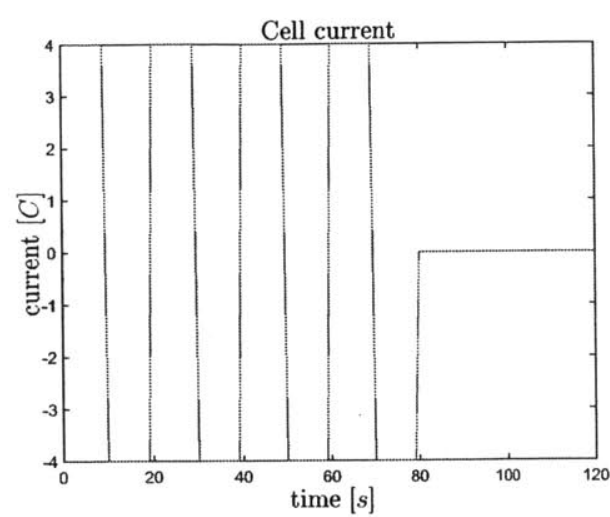

Figure 4-18: A series of charges and discharges at $4 \mathrm{C}$

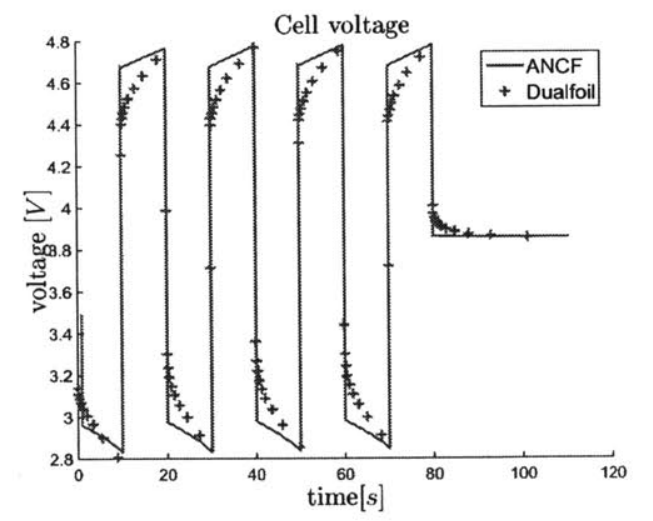

Figure 4-19: Cell voltage for the 4C discharge and charge cycle

\subsubsection{Comparison of the ANCF model to the SPM}

In this section, we compare the ANCF model with the single particle model (SPM) [2] on a $\mathrm{LiCoO}_{2} / \mathrm{LiC}_{6}$ cell. We will evaluate two cases. In the first case, a constant discharge with $1 C\left(30 \mathrm{~A} / \mathrm{m}^{2}\right)$ is simulated. For the second simulation a pulse discharge with $1 C$ for 600 seconds will be analyzed. Furthermore, we simulate the ANCF models with increasing numbers of elements in the electrodes. The number of nodes is not increased in the separator, because of the well-behaved profiles within it. A finite difference model of the P2D formulation is used as reference.

The voltages over time are compared. For the first case, the ANCF model perfectly approaches the finite difference model for three nodes/two elements in the electrodes as displayed in Fig.(4-21). If we choose only one element, the absolute nodal coordinate polynomial is not able to capture the late phase of the discharge, when the solid concentration in the negative electrode is low. At this point the polynomial approximates the solid concentration to values of zero too early. The single particle model does not include any transport phenomena in the electrolyte and thus neglects the loss induced by them. Hence, the SPM will always display a higher power capacity/voltage than is available in reality. This difference can be clearly seen in the case of a pulse discharge of $1 C$ between $t=200 \mathrm{~s}$ and $t=800 \mathrm{~s}$ in Fig.(4-22) and 


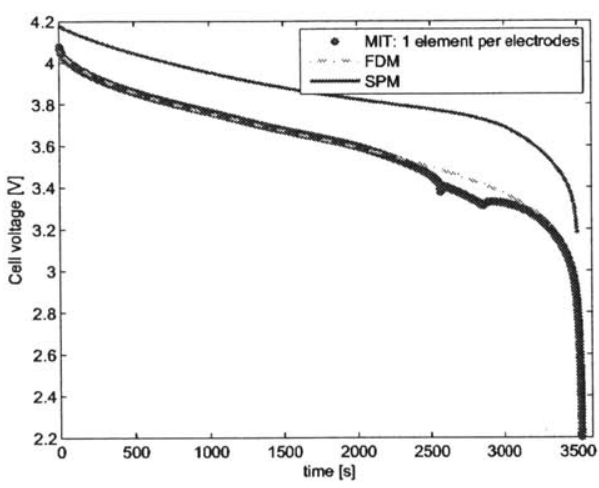

Figure 4-20: 1C discharge comparison: ANCF model with one element per electrode, FDM and SPM



Figure 4-22: Pulse discharge between $t=$ $200 s$ and $t=800 s$ : ANCF model with one element per electrode, FDM and SPM

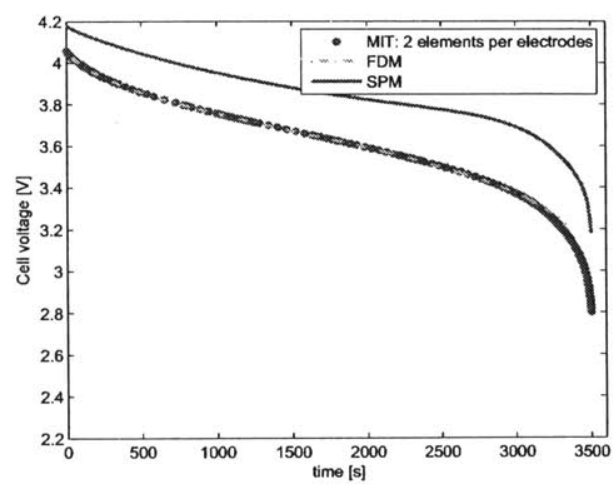

Figure 4-21: $1 \mathrm{C}$ discharge comparison: ANCF model with two elements per electrode, FDM and SPM

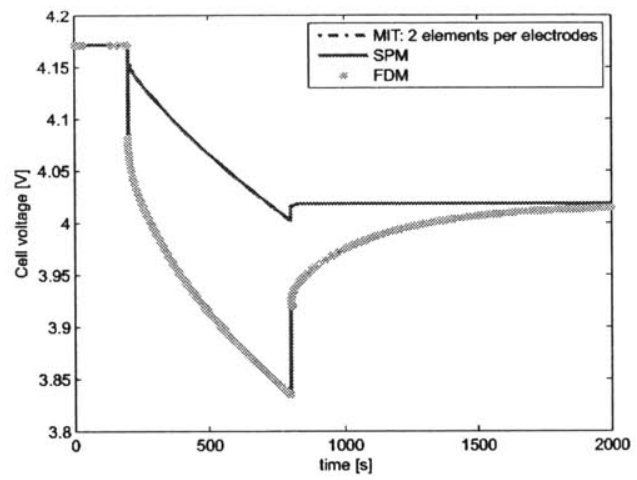

Figure 4-23: Pulse discharge between $t=$ $200 s$ and $t=800 s$ : ANCF model with two elements per electrode, FDM and SPM

Fig.(4-21). We conclude that for the system presented two nodes per electrodes are sufficient to converge to the solution of the finite difference method.

\subsubsection{Extended adaptive observer on a ANCF model plant}

We now proceed to estimate parameters of a $\mathrm{LiCoO}_{2} / \mathrm{LiC}_{6}$ cell, using the adaptive observer described in Section 3. We simulate the observer on the ANCF model for low amplitude input current shown in fig.4-24, at which reactions kinetics don't reach into the Tafel region. The plant is subjected to a superposition of harmonic inputs, 
which creates a persistently exciting signal. Initial states of the plant are $S O C_{a}=0.8$ and $S O C_{c}=0.28$ and the cell starts from a state of equilibrium. Initial conditions of the estimated parameters are chosen to be off by $60 \%$. The initial estimate of the cell states have a $10 \%$ error. Filter values of the observer are chosen to be near the initial estimates for plant poles as $\lambda_{1}=10^{-2}, \lambda_{2}=10^{-3}$ and $\lambda_{3}=10^{-4}$. The optimal choice of filter values is a topic of on-going research. Initial gain for the recursive least square algorithm is chosen to be $\Gamma(0)=10^{6} \mathbf{I}$ and the forgetting factor is $q=10^{-12}$. Parameter estimates usually converge within $5 \%$ error boundaries after $10^{\prime} 000 \mathrm{~s}$ as shown in fig.4-26. Although the output error $e=y_{p}-\hat{y}$ does not entirely converge to zero, due to the nonlinearities in the system, the estimated plant matches well the real plant. This can inferred by comparing the pole and zero plots of the real plant linearized at initial conditions and the estimated plant pole and zeros at $t=0$ in fig.4-27 and at $t=10^{\prime} 000 \mathrm{~s}$ in fig.4-28.

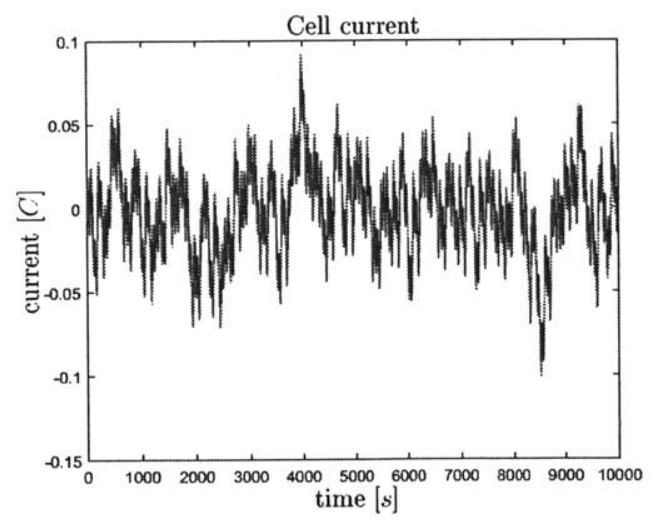

Figure 4-24: Superposed sinusoidal signals as input current

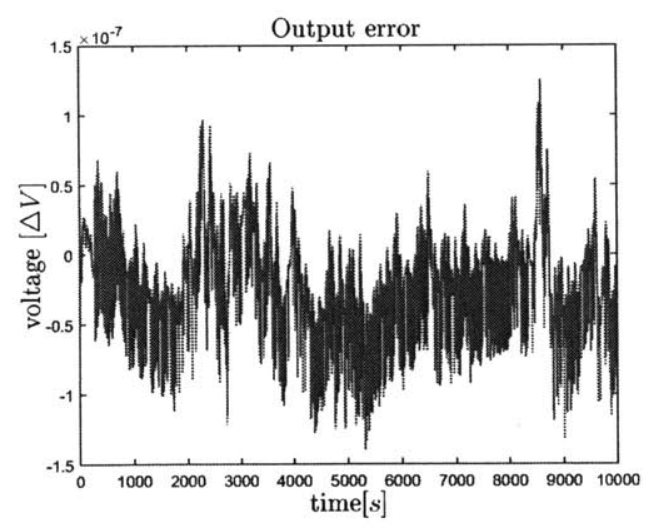

Figure 4-25: Error between estimated cell voltage and real cell voltage 


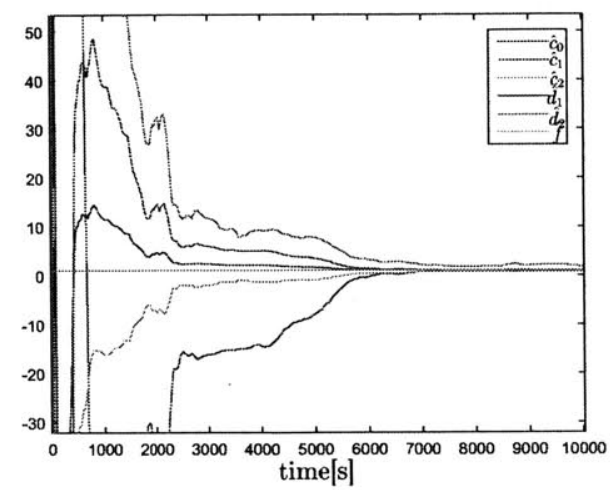

Figure 4-26: Estimated parameters normalized by the actual plant parameter values

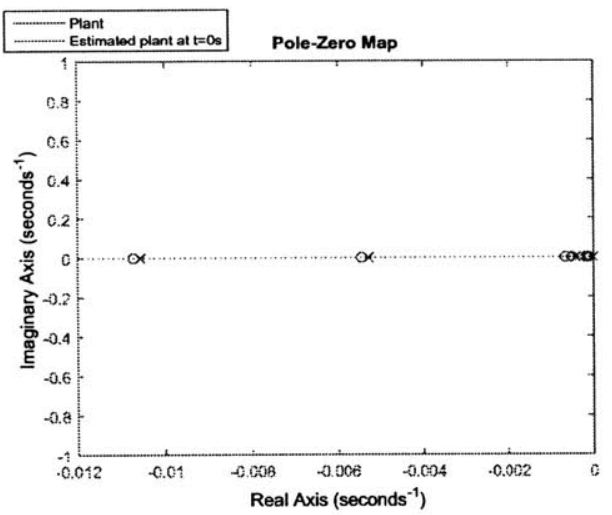

Figure 4-27: Pole/Zero plots compared for initial conditions

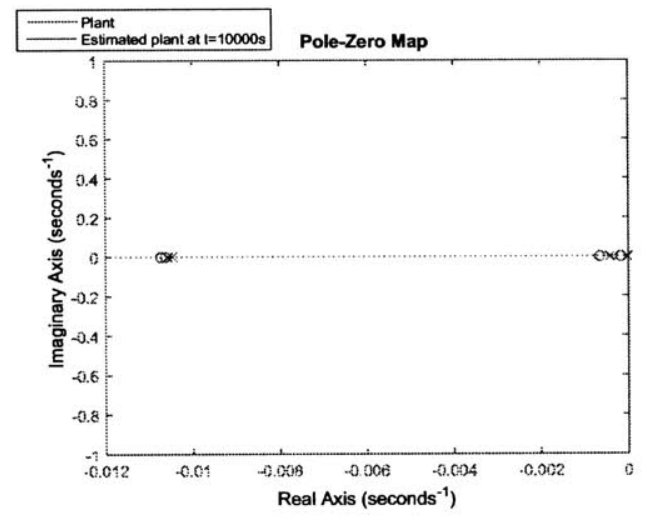

Figure 4-28: Pole/Zero plots after $10^{\prime} 000 s$ 


\section{Chapter 5}

\section{Conclusion}

Driving down costs of electric vehicles, is of great importance, if they are supposed to be the principal mean of transportation for the future. While lithium-ion technology is a very attractive option for energy storage, its rareness makes it very expensive and the cost-driving component of an all electric drive vehicle. Thus, enabling efficient use of Li-ion batteries, could lead to significantly reduced costs and therefore, represents one of the pathways towards a more sustainable future.

Battery management systems (BMS) enable such efficient use of batteries. One vital part of a BMS is the monitoring unit, which estimates state of health ( $\mathrm{SOH})$ and state of charge (SOC) of the cell at any given time. Research on this unit, has been picked up by academia and industry in recent years. Estimation algorithms based on different types of models have been explored. In this thesis, we focused our work on estimation algorithms based on electrochemical models, which as shown in chapter 4 are based on the physical properties of the li-ion cell and can be precise predictors for cell behavior in any given operation mode. We've presented two new findings, which could improve the precision and performance of such a monitoring unit. The first one is an extended adaptive observer for Li-ion cells and the second one is a reduced order model of the well-known porous electrode model [5].

Electrochemical models used for battery monitoring have been mainly based on the 
single particle model. We relied on the same model as plant description and applied a new extended adaptive observer to the plant. The extended adaptive observer is based on a linear non-minimal representation of the plant and is applicable to plants with proper transfer functions. Parameters and states can be estimated simultaneously with this observer and for application to linear plants, tracking of states has been proven by the appropriate choice of Lyapunov function. Parameter convergence can be guaranteed in case of persistently exciting input signals. The adaptive observer is shown to result in accurate parameter estimation of a $\mathrm{LiCOO}_{2} / \mathrm{LiC}_{6}$ cell at low input currents. The same observer can also be applied to higher order models, as shown for a two cathode material cell, based on the a $\mathrm{LiCOO}_{2} / \mathrm{LiC}_{6}$ chemistry. Nonlinearities in the output, limit the application of the presented observer to low input currents, where reaction kinetics don't reach the Tafel region. To reliably estimate states and parameters under any operating condition, observers, which account for the nonlinearities, will have to be developed.

Although, the SPM is derived from the highly accurate porous electrode model [13], it cannot reliably capture internal dynamics of batteries subjected to EV drive cycles as shown in section 4.4.1. Therefore, we introduced a new reduced-order model based on ANCF and showed it's capability of replicating the cell behavior for high input currents. The derivation relies on the absolute nodal coordinate formulation, a method first introduced in beam theory [28]. The cell is discretized in a variable number of elements. First principle equations for each of these elements are then approximated by third order polynomials, whose coefficients describe the states and their spatial derivatives at each nodes. Mathematical approximation is performed by applying Galerkin projections to each element, also known as the method of weighted residuals. The resulting ANCF model was validated by subjecting it to two charging and discharging scenarios with current amplitudes up to four Cs for a $\mathrm{LiCOO}_{2} / \mathrm{LiC}_{6}$ cell. Moreover, the ANCF model was used as high fidelity model for parameter estimation based on an SPM model. We applied the above mentioned extended adaptive observer to the ANCF model for a persistently exciting input current, consisting of a 
superposition of sinusoids. Parameter estimates converged after $10000 \mathrm{~s}$ and remained in a $5 \%$ error boundary. Future modifications to the ANCF model might be made for varying diffusion coefficient. In cases of extremely small electrolyte diffusion coefficients, the ANCF model might be not appropriate as the number of elements has to be increased dramatically or their lengths have to varied. A better approximation could be potentially achieved if the diffusion coefficient is varied per element depending on the average salt concentration in the element. 


\section{Appendix A}

\section{A.1 Notations}

\begin{tabular}{|l|l|}
\hline \multicolumn{2}{|l|}{ Variables } \\
\hline$x$ & Spatial variable for cell \\
$r$ & Pseudo variable in solid electrode particle \\
$t$ & Time \\
$T$ & Temperature \\
$c_{e}$ & Li-ion concentration in electrolyte \\
$\phi_{e}$ & Potential in electrolyte \\
$c_{s}$ & Li-ion concentration in solid electrode \\
$\phi_{s}$ & Potential in solid electrode \\
$j_{n}$ & Molar flux at the surface of the electrode material \\
$\bar{c}_{s}$ & Volume averaged Li-ion concentration in solid electrode \\
$\overline{j_{s}}$ & Volume averaged molar flux in the solid electrode \\
$c_{s s}$ & Surface concentration of the solid electrode \\
$I$ & Applied cell current \\
$V$ & Cell output voltage \\
\hline Subscripts \\
\hline$(\bullet)^{+}$ & Addressing positive cathode material \\
$(\bullet)^{-}$ & Addressing negative cathode material \\
$(\bullet)_{1}$ & Addressing cathode material 1 \\
$(\bullet)_{2}$ & Addressing cathode material 2 \\
\hline
\end{tabular}

Table A.1: Variables and subscript notations 


\begin{tabular}{|l|l|l|}
\hline \multicolumn{2}{|l|}{ Parameters } & Units \\
\hline Notation & Definition & $\frac{1}{m}$ \\
\hline$a^{-}$ & Anode's specific interfacial area & $\frac{1}{m}$ \\
$a_{1}^{+}$ & Cathode's specific interfacial area & $\frac{1}{m}$ \\
$a_{2}^{+}$ & Cathode's specific interfacial area & $\frac{m^{2}}{s}$ \\
$D_{s}^{-}$ & Effective solid electrode diffusion coefficient & $\frac{m^{2}}{s}$ \\
$D_{s, 1}^{+}$ & Effective solid electrode diffusion coefficient & $\frac{m^{2}}{s}$ \\
$D_{s, 2}^{+}$ & Effective solid electrode diffusion coefficient & $\frac{m o l}{s m^{2}} \frac{m o l}{m^{3}}$ \\
$r_{e f f}^{-}$ & Reaction constant of anode \\
$r_{e f f, 1}^{+}$ & Reaction constant of cathode material 1 & $\frac{m o l}{s m^{2}} \frac{m o l}{m^{3}}$ \\
$r_{e f f, 2}^{+}$ & Reaction constant of cathode material 2 & $\frac{m o l}{s m^{2}} \frac{m o l}{m^{3}}$ \\
$R_{p}^{-}$ & Porous anode electrode pseudo particle radius & $m$ \\
$R_{p, 1}^{+}$ & Porous cathode electrode pseudo particle radius & $m$ \\
$R_{p, 2}^{+}$ & Porous cathode electrode pseudo particle radius & $m$ \\
$L^{-}$ & Anode compartment length & $m$ \\
$L^{+}$ & Cathode compartment length & $m$ \\
$R_{f}^{-}$ & Solid-electrolyte interphase resistivity & $\Omega m^{2}$ \\
$R_{f, 1}^{+}$ & Solid-electrolyte interphase resistivity & $\Omega m^{2}$ \\
$R_{f, 2}^{+}$ & Solid-electrolyte interphase resistivity & $\Omega m^{2}$ \\
$c_{s, m a x}^{-}$ & Maximum Li-ion concentration in solid electrode & $\frac{m o l}{m^{3}}$ \\
$c_{s, m a x, 1}^{+}$ & Maximum Li-ion concentration in solid electrode & $\frac{m o l}{m^{3}}$ \\
$c_{s, m a x, 2}^{+}$ & Maximum Li-ion concentration in solid electrode & $\frac{m o l}{m^{3}}$ \\
$c_{e}^{0}$ & Initial electrolyte concentration & $\frac{m o l}{m^{3}}$ \\
$\mathcal{U}^{-}$ & OCV of anode & $V$ \\
$\mathcal{U}_{1}^{+}$ & OCV of cathode 1 & $V$ \\
$\mathcal{U}_{2}^{+}$ & OCV of cathode 2 & $V$ \\
\hline
\end{tabular}

Table A.2: Variables, Parameters and subscript notations 


\section{A.2 Parameter values}

\begin{tabular}{|c|c|c|c|}
\hline Notation & Definition & Units & Value \\
\hline$a^{-}$ & Anode's specific interfacial area & $\frac{1}{m}$ & $7.2360 \mathrm{e}+05$ \\
\hline$a^{+}$ & Cathode's specific interfacial area & $\frac{1}{m}$ & 885000 \\
\hline$D_{s}^{-}$ & Effective solid electrode diffusion coefficient & $\frac{m^{2}}{s}$ & $1 e-14$ \\
\hline$D_{s}^{+}$ & Effective solid electrode diffusion coefficient & $\frac{m^{2}}{s}$ & $3.9 e-14$ \\
\hline$k^{-}$ & Reaction constant of anode & $\frac{m o l}{s m^{2}}{\frac{m o l}{m^{3}}}^{1+\alpha a, i}$ & $5.0307 \mathrm{e}-11$ \\
\hline$k^{+}$ & Reaction constant of cathode material 1 & $\frac{m o l}{s m^{2}}{\frac{m o l}{m^{3}}}^{1+\alpha a, i}$ & $2.334 \mathrm{e}-11$ \\
\hline$R_{p}^{-}$ & Porous anode electrode pseudo particle radius & $m$ & $2 \mathrm{e}-6$ \\
\hline$R_{p}^{+}$ & Porous cathode electrode pseudo particle radius & $m$ & $2 \mathrm{e}-6$ \\
\hline$L^{-}$ & Anode compartment length & $m$ & $88 e-6$ \\
\hline$L^{+}$ & Cathode compartment length & $m$ & $80 e-6$ \\
\hline$L^{s e p}$ & Separator compartment length & $m$ & $25 \mathrm{e}-6$ \\
\hline$R_{S E I}^{-}$ & Solid-electrolyte interphase resistivity & $\Omega m^{2}$ & 0 \\
\hline$R_{S E I}^{+}$ & Solid-electrolyte interphase resistivity & $\Omega m^{2}$ & 0 \\
\hline$c_{s, \max }^{-}$ & Maximum Li-ion concentration in solid electrode & $\frac{m o l}{m^{3}}$ & 30555 \\
\hline$c_{s, \max }^{+}$ & Maximum Li-ion concentration in solid electrode & $\frac{m o l}{m^{3}}$ & 51554 \\
\hline$c_{e}^{0}$ & Initial electrolyte concentration & $\frac{m o l}{m^{3}}$ & 1000 \\
\hline$D_{e f f}$ & Effective electrolyte diffusion coefficient & $\frac{m^{2}}{s}$ & $D_{e f f}=D \epsilon_{e}^{b r u g g}$ \\
\hline$D$ & Electrolyte diffusion coefficient & $\frac{m^{2}}{s}$ & $7.5 \mathrm{e}-10$ \\
\hline brugg & Brugg coefficient & - & 4 \\
\hline
\end{tabular}

Table A.3: $\mathrm{LiCoO}_{2} / \mathrm{LiC}_{6}$ : List of parameters 


\begin{tabular}{|l|l|l|l|}
\hline Notation & Definition & Units & Value \\
\hline$\epsilon_{e}^{-}$ & Electrolyte volume fraction & - & 0.485 \\
$\epsilon_{e}^{\text {sep }}$ & Electrolyte volume fraction & - & 0.724 \\
$\epsilon_{e}^{+}$ & Electrolyte volume fraction & - & 0.385 \\
$\sigma_{e f f}$ & Effective electrode electric conductivity & $\frac{S}{m}$ & $\sigma_{e f f}$ \\
& & & $=\sigma\left(1-\epsilon_{e}-\epsilon_{f}\right)$ \\
$\sigma^{-}$ & Electrode electric conductivity & $\frac{S}{m}$ & 100 \\
$\sigma^{+}$ & Electrode electric conductivity & $\frac{S}{m}$ & 100 \\
$\epsilon_{f}^{-}$ & Binding material volume fraction & - & 0.0326 \\
$\epsilon_{f}^{+}$ & Binding material volume fraction & - & 0.025 \\
$\kappa_{e f f}$ & Effective electrolyte ionic conductivity & $\frac{S}{m}$ & $\kappa_{e f f}=\kappa \epsilon_{e}^{b r u g g}$ \\
$\kappa$ & Electrolyte ionic conductivity & $\frac{S}{m}$ & 0.3 \\
$t_{a}^{0}$ & Transference number of anions & - & 0.637 \\
$t_{c}^{0}$ & Transference number of cations & - & 0.363 \\
$T$ & Temperature & $\mathrm{K}$ & 297.15 \\
$\alpha^{a / c}$ & Anion/cation transfer coefficient & - & 0.5 \\
$\mathcal{U}^{-}$ & OCV of anode & $V$ & - \\
$\mathcal{U}^{+}$ & OCV of cathode & $V$ & - \\
\hline
\end{tabular}

Table A.4: $\mathrm{LiCoO}_{2} / \mathrm{LiC}_{6}$ : List of parameters

\section{A.3 Input signal}

The input signals for parameter estimation in 3.2 .5 were chosen to be superpositions of sinusoid functions

$$
I(t)=\sum_{i=1}^{n} I_{a}(-1)^{i} \sin \left(f_{i} t\right) .
$$


For the simulation on the linear SPM plant, we choose

$$
\begin{gathered}
I_{a}=1\left[\mathrm{~A} / \mathrm{m}^{2}\right] \text { and } \mathbf{f}=\left[10^{-5}, 5 \times 10^{-5}, 10^{-4}, 2 \times 10^{-4}, 3 \times 10^{-4}, 4 \times 10^{-4},\right. \\
4.96 \times 10^{-4}, 5 \times 10^{-4}, 1 \times 10^{-3}, 1.5 \times 10^{-3}, 1.6 \times 10^{-3}, 5 \times 10^{-3}, 1 \times 10^{-2}, \\
\left.1.51 \times 10^{-2}, 2 \times 10^{-2}, 3.52 \times 10^{-2}, 5 \times 10^{-2}, 1 \times 10^{-1}, 1\right][\mathrm{rad} / \mathrm{s}]
\end{gathered}
$$

For the simulation on the nonlinear SPM plant and the ANCF plant, we choose

$$
\begin{gathered}
I_{a}=0.1\left[\mathrm{~A} / \mathrm{m}^{2}\right] \text { and } \mathbf{f}=\left[10^{-5}, 5 \times 10^{-5}, 10^{-4}, 2 \times 10^{-4}, 3 \times 10^{-4}, 4 \times 10^{-4},\right. \\
4.96 \times 10^{-4}, 5 \times 10^{-4}, 1 \times 10^{-3}, 1.5 \times 10^{-3}, 1.6 \times 10^{-3}, 5 \times 10^{-3}, 1 \times 10^{-2}, \\
\left.1.51 \times 10^{-2}, 2 \times 10^{-2}, 3.52 \times 10^{-2}, 5 \times 10^{-2}, 1 \times 10^{-1}, 1\right][\mathrm{rad} / \mathrm{s}]
\end{gathered}
$$

For the simulation on the linear two cathode material plant, we choose

$$
\begin{gathered}
I_{a}=1\left[\mathrm{~A} / \mathrm{m}^{2}\right] \text { and } \mathbf{f}=\left[10^{-5}, 5 \times 10^{-5}, 10^{-4}, 2 \times 10^{-4}, 3 \times 10^{-4}, 4 \times 10^{-4},\right. \\
4.96 \times 10^{-4}, 5 \times 10^{-4}, 1 \times 10^{-3}, 1.5 \times 10^{-3}, 1.6 \times 10^{-3}, 5 \times 10^{-3}, 1 \times 10^{-2}, \\
\left.1.51 \times 10^{-2}, 2 \times 10^{-2}, 3.52 \times 10^{-2}, 5 \times 10^{-2}, 1 \times 10^{-1}, 1\right][\mathrm{rad} / \mathrm{s}]
\end{gathered}
$$




\section{Bibliography}

[1] Jian Cao, Nigel Schofield, and Ali Emadi. Battery balancing methods: A comprehensive review. In Vehicle Power and Propulsion Conference, 2008. VPPC'08. IEEE, pages 1-6. IEEE, 2008.

[2] N.A. Chaturvedi, R. Klein, J. Christensen, J. Ahmed, and A. Kojic. Algorithms for advanced battery-management systems. Control Systems, IEEE, 30(3):49 68, 2010 .

[3] Nalin A Chaturvedi, Jake F Christensen, Reinhardt Klein, and Aleksandar Kojic. Approximations for partial differential equations appearing in li-ion battery models. In ASME 2013 Dynamic Systems and Control Conference, pages V001T05A004-V001T05A004. American Society of Mechanical Engineers, 2013.

[4] Domenico Di Domenico, Eric Prada, and Yann Creff. An adaptive strategy for li-ion battery soc estimation. In Proceedings of 2011 IFAC World Congress, 2011.

[5] Marc Doyle, Thomas F. Fuller, and John Newman. Modeling of galvanostatic charge and discharge of the lithium/polymer/insertion cell. Journal of The Electrochemical Society, 140(6):1526-1533, 1993.

[6] John B. Goodenough and Youngsik Kim. Challenges for rechargeable li batteriesâĂă. Chemistry of Materials, 22(3):587-603, 2010.

[7] Petros A Ioannou and Jing Sun. Robust adaptive control. Courier Dover Publications, 2012.

[8] Gerhard Kreisselmeier. Adaptive observers with exponential rate of convergence. Automatic Control, IEEE Transactions on, 22(1):2-8, 1977.

[9] Yuang-Shung Lee and Ming-Wang Cheng. Intelligent control battery equalization for series connected lithium-ion battery strings. Industrial Electronics, IEEE Transactions on, 52(5):1297-1307, 2005.

[10] Languang Lu, Xuebing Han, Jianqiu Li, Jianfeng Hua, and Minggao Ouyang. A review on the key issues for lithium-ion battery management in electric vehicles. Journal of Power Sources, 226:272-288, 2013. 
[11] SJ Moura, NA Chaturvedi, and M Krstic. Pde estimation techniques for advanced battery management systemsŮpart i: Soc estimation. In American Control Conference (ACC), 2012, pages 559-565. IEEE, 2012.

[12] Kumpati S Narendra and Anuradha M Annaswamy. Stable adaptive systems. Courier Dover Publications, 2012.

[13] J. Newman and K.E. Thomas-Alyea. Electrochemical Systems. Electrochemical Society series. Wiley, 2004.

[14] Paul WC Northrop, Venkatasailanathan Ramadesigan, Sumitava De, and Venkat $R$ Subramanian. Coordinate transformation, orthogonal collocation, model reformulation and simulation of electrochemical-thermal behavior of lithium-ion battery stacks. Journal of The Electrochemical Society, 158(12):A1461-A1477, 2011.

[15] Habiballah Rahimi-Eichi, Unnati Ojha, Federico Baronti, and M Chow. Battery management system: an overview of its application in the smart grid and electric vehicles. Industrial Electronics Magazine, IEEE, 7(2):4-16, 2013.

[16] Saeed Khaleghi Rahimian, Sean Rayman, and Ralph E White. State of charge and loss of active material estimation of a lithium ion cell under low earth orbit condition using kalman filtering approaches. Journal of The Electrochemical Society, 159(6):A860-A872, 2012.

[17] Venkatasailanathan Ramadesigan, Paul W. C. Northrop, Sumitava De, Shriram Santhanagopalan, Richard D. Braatz, and Venkat R. Subramanian. Modeling and simulation of lithium-ion batteries from a systems engineering perspective. Journal of The Electrochemical Society, 159(3):R31-R45, 2012.

[18] Shriram Santhanagopalan and Ralph E White. Online estimation of the state of charge of a lithium ion cell. Journal of power sources, 161(2):1346-1355, 2006.

[19] Alexander P. Schmidt, Matthias Bitzer, Ã?rpÃąd W. Imre, and Lino Guzzella. Experiment-driven electrochemical modeling and systematic parameterization for a lithium-ion battery cell. Journal of Power Sources, 195(15):5071 - 5080, 2010.

[20] Venkat R Subramanian, Vijayasekaran Boovaragavan, Venkatasailanathan Ramadesigan, and Mounika Arabandi. Mathematical model reformulation for lithium-ion battery simulations: Galvanostatic boundary conditions. Journal of The Electrochemical Society, 156(4):A260-A271, 2009.

[21] Venkat R. Subramanian, Vinten D. Diwakar, and Deepak Tapriyal. Efficient macro-micro scale coupled modeling of batteries. Journal of The Electrochemical Society, 152(10):A2002 A2008, 2005.

[22] The New York Times. Car fire a test for high-flying tesla, 2013. 
[23] Wladislaw Waag, Christian Fleischer, and Dirk Uwe Sauer. Critical review of the methods for monitoring of lithium-ion batteries in electric and hybrid vehicles. Journal of Power Sources, 258:321-339, 2014.

[24] Shuoqin Wang, Mark Verbrugge, Luan Vu, Daniel Baker, and John S Wang. Battery state estimator based on a finite impulse response filter. Journal of The Electrochemical Society, 160(11):A1962-A1970, 2013.

[25] Shuoqin Wang, Mark Verbrugge, John S Wang, and Ping Liu. Multi-parameter battery state estimator based on the adaptive and direct solution of the governing differential equations. Journal of Power Sources, 196(20):8735-8741, 2011.

[26] Yebin Wang, Huazhen Fang, Zafer Sahinoglu, Toshihiro Wada, and Satoshi Hara. Nonlinear adaptive estimation of the state of charge for lithium-ion batteries. In Decision and Control (CDC), 2013 IEEE 52nd Annual Conference on, pages 4405-4410. IEEE, 2013.

[27] Yinjiao Xing, Eden WM Ma, Kwok L Tsui, and Michael Pecht. Battery management systems in electric and hybrid vehicles. Energies, 4(11):1840-1857, 2011.

[28] Refaat Y Yakoub and Ahmed A Shabana. Three dimensional absolute nodal coordinate formulation for beam elements: implementation and applications. Journal of Mechanical Design, 123:614, 2001. 